University of Tennessee Health Science Center

UTHSC Digital Commons

\title{
Generation and Characterization of a Knock-In Allele of EKLF: Probing the in vivo Role of the Chromatin Remodeling Domain in Definitive Hematopoietic Cells
}

Valerie Malyvanh Jansen

University of Tennessee Health Science Center

Follow this and additional works at: https://dc.uthsc.edu/dissertations

Part of the Medical Molecular Biology Commons

\section{Recommended Citation}

Jansen, Valerie Malyvanh, "Generation and Characterization of a Knock-In Allele of EKLF: Probing the in vivo Role of the Chromatin Remodeling Domain in Definitive Hematopoietic Cells" (2009). Theses and Dissertations (ETD). Paper 139. http://dx.doi.org/10.21007/etd.cghs.2009.0152. 


\title{
Generation and Characterization of a Knock-In Allele of EKLF: Probing the in vivo Role of the Chromatin Remodeling Domain in Definitive Hematopoietic Cells
}

\begin{abstract}
The zinc finger-encoding transacting factor EKLF, or erythroid Krüppel-like factor, binds key regulatory elements of many erythroid-specific genes, and is essential for definitive erythropoiesis. Mice lacking this factor die of anemia by E15.5 of gestation, failing to activate $\beta$-globin gene transcription, and demonstrating a block in the erythroid differentiation program at the primitive erythroblast stage. In contrast, megakaryocytic progenitors are amplified in EKLF-null embryos, with increased Fli- 1 gene expression, a marker of early megakaryocytic differentiation. These observations are consistent with the idea that EKLF modulates the megakaryocytic-erythroid (M-E) differentiation switch.

Our laboratory has previously demonstrated that an amino terminal sequence of EKLF (D221EKLF) is required to induce chromatin remodeling at the $\beta$-globin promoter in an EKLF-null erythroid cell line. However, additional amino terminal sequences are required for initiation of $\beta$-globin gene transcription. To evaluate the role of this chromatin remodeling domain in erythroid and megakaryocytic differentiation in vivo, I have generated a knock-in allele of D221EKLF. Using the recombineering method, a lambda phagebased homolgous recombination method in E. coli, cDNA encoding theD221EKLF domain has been inserted into the endogenous initiation site, thus placing the mutant protein under the cis-regulatory elements of the endogenous murine EKLF locus. Subsequently, D221EKLF alleles have been generated by gene targeting in ES cells. I have used the mice to probe the in vivo role of D221EKLF in definitive hematopoietic cells.

Similar to EKLF-null embryos, mice homozygous for the D221EKLF mutant allele die of anemia by E15.5 of gestation. Molecular analysis ofD221EKLF erythroblasts reveals i) a failure to activate $\beta$-globin gene transcription; ii) lack of GATA-1 and NF-E2 recruitment to the $\beta$-globin promoter; iii) a block in terminal erythroid differentiation. In contrast to erythroid cells lacking EKLF, D221EKLF erythroid progenitors demonstrate appropriate binding of the D221EKLF encoding domain to all EKLF-regulatory sequences and a chromatin architecture and histone modification pattern at erythroid-specific genes that recapitulate the events observed in wild-type EKLF erythroblasts at a similar stage of erythroid ontogeny.

Examining the role of D221EKLF in megakaryopoiesis, I observed inhibition of megakaryocytic progenitor expansion in D221EKLF fetal hematopoietic cell populations when compared to EKLF-null embryos. Molecular analysis of D221EKLF erythroblasts reveals i) binding of theD221EKLF mutant protein to the Fli-1 promoter with inhibition of gene transcription; ii) hypoacetylation of histone $\mathrm{H} 3$ at the Fli-1 promoter; iii) recruitment of a Sin3A-containing corepressor complex to the Fli- 1 promoter. Taken together, my results suggest strongly that the unique D221EKLF domain is sufficient to modulate the chromatinspecific roles of EKLF at erythroid- and megakaryocytic-specific loci in definitive hematopoietic cells in vivo.
\end{abstract}

Document Type

Dissertation

Degree Name

Doctor of Philosophy (PhD)

Program

Molecular Sciences 


\section{Research Advisor}

John M. Cunningham, M.D.

\section{Keywords}

B-globin, chromatin, EKLF, erythropoiesis, megakaryopoiesis

\section{Subject Categories}

Medical Molecular Biology | Medical Sciences | Medicine and Health Sciences 
GENERATION AND CHARACTERIZATION OF A KNOCK-IN ALLELE OF EKLF: PROBING THE IN VIVO ROLE OF THE CHROMATIN REMODELING DOMAIN IN DEFINITIVE HEMATOPOIETIC CELLS

\author{
A Dissertation \\ Presented for \\ The Graduate Studies Council \\ The University of Tennessee \\ Health Science Center

\begin{abstract}
In Partial Fulfillment
Of the Requirements for the Degree

Doctor of Philosophy

From The University of Tennessee
\end{abstract}

By

Valerie Malyvanh Jansen

May 2009 
Copyright (C) 2009 by Valerie Malyvanh Jansen All rights reserved 


\section{DEDICATION}

This dissertation is dedicated in the loving memory of Laura Jeanne Jansen who saw me begin this journey and is watching from above as I finish.

To my husband, Tim, for his unwavering love and support throughout this journey.

To my precious daughter, Hannah, the absolute best result during this journey.

The journey is the reward.

Chinese Proverb 


\section{ACKNOWLEDGMENTS}

I would like to express my deepest gratitude to those who have contributed in many different ways to my dissertation research over the past years. First and foremost, I thank my research advisor, Dr. John Cunningham, for the opportunity to train in his laboratory and for his encouragement and unconditional support. He has been a great mentor and role model in many aspects of my development as a future physician scientist. I would also like to acknowledge all the past and present members of the Cunningham laboratory for their friendship and moral and scientific support: Aurelie Desgardin, Vishwas Parekh, Eun-Hee Shim, Sree Kartha, Xiaing Tian, Shaji Ramachandran, and Bala Poonkuzhali. I would like to thank Xiuling Li at St Jude Children's Research Hospital for her outstanding technical expertise and assistance with ES cell targeting. I would also like to thank Tatiana Abramova for her technical assistance with chromatin immunoprecipitation in the final months of my research and for her dear friendship.

I also extend my deepest appreciation to my graduate committee, Dr. S. Ken Nishimoto, Dr. Gerard Zambetti, Dr. Linda Hendershot, and Dr. Derek Persons for their guidance, support, and commitment, especially during my time of transition to the University of Chicago. I would also like to acknowledge the various people whom I have met and interacted with at St Jude in the Department of Hematology and Division of Experimental Hematology. It is without a doubt that their friendship, mentorship, and support over the years have had a part in where I am today and the physician, scientist, and human being I aspire to be in the future. I particularly would like to acknowledge Dr. Winfred Wang, Dr. Jane Hankins, Dr. Maggie Evans-Galea, Dr. Joan Chesney, Dr. Jennifer Hockings, Dr. Ted Hofmann, and Phillip Hargrove. Special thanks to my good friend Nick Phillips for connecting me with the right mentors and for all the "pep talks" when I needed them most.

I would also like to acknowledge the individuals at UTHSC that made it possible for me to transition from medical school to the graduate program in Molecular Sciences and for their immense support when I moved to Chicago: Dr. Pat Ryan, Dr. Ed Schneider, Janie Van Prooijen, Sue Farmer, and Dr. Richard Peppler, now at the University of Central Florida School of Medicine. I am also thankful to Dr. Don Thomason for his immense support at the end of my dissertation.

I am grateful for the wonderful new friends and colleagues I have met at the University of Chicago, especially the members of Dr. Susan Cohn's laboratory and my fellow Pritzker medical students in the class of 2010.

Last, but certainly not least, I would like to acknowledge my family because without them none of this would have been possible. My husband, Tim, for his unconditional love and support that allowed me to pursue my dreams and goals. I promise the end is in sight! My precious daughter, Hannah, for bringing me great joy and happiness and for understanding that "mommy goes to the lab while I go to preschool." I hope she'll remember the great times we had together rather than "mommy being mean 
for leaving and not going to sleep." I am forever grateful and blessed to have my fatherin-law, Rick Jansen, who was always ready and willing to help with Hannah. I am thankful to my sister, Phoukhaothong Vichidvongsa, for her encouragement and support as I finished. Finally, I am thankful to my parents for instilling in me the importance of education, for their hard work and struggles to provide me with the best opportunities in life, and for their unconditional love and support. 


\begin{abstract}
The zinc finger-encoding transacting factor EKLF, or erythroid Krüppel-like factor, binds key regulatory elements of many erythroid-specific genes, and is essential for definitive erythropoiesis. Mice lacking this factor die of anemia by E15.5 of gestation, failing to activate $\beta$-globin gene transcription, and demonstrating a block in the erythroid differentiation program at the primitive erythroblast stage. In contrast, megakaryocytic progenitors are amplified in EKLF-null embryos, with increased Fli-1 gene expression, a marker of early megakaryocytic differentiation. These observations are consistent with the idea that EKLF modulates the megakaryocytic-erythroid (M-E) differentiation switch.

Our laboratory has previously demonstrated that an amino terminal sequence of EKLF $(\triangle 221 \mathrm{EKLF})$ is required to induce chromatin remodeling at the $\beta$-globin promoter in an EKLF-null erythroid cell line. However, additional amino terminal sequences are required for initiation of $\beta$-globin gene transcription. To evaluate the role of this chromatin remodeling domain in erythroid and megakaryocytic differentiation in vivo, I have generated a knock-in allele of $\triangle 221 \mathrm{EKLF}$. Using the recombineering method, a lambda phage-based homolgous recombination method in E. coli, cDNA encoding the $\triangle 221 \mathrm{EKLF}$ domain has been inserted into the endogenous initiation site, thus placing the mutant protein under the cis-regulatory elements of the endogenous murine EKLF locus. Subsequently, $\triangle 221 \mathrm{EKLF}$ alleles have been generated by gene targeting in ES cells. I have used the mice to probe the in vivo role of $\Delta 221 \mathrm{EKLF}$ in definitive hematopoietic cells.
\end{abstract}

Similar to EKLF-null embryos, mice homozygous for the $\triangle 221 \mathrm{EKLF}$ mutant allele die of anemia by E15.5 of gestation. Molecular analysis of $\triangle 221 \mathrm{EKLF}$ erythroblasts reveals i) a failure to activate $\beta$-globin gene transcription; ii) lack of GATA1 and NF-E2 recruitment to the $\beta$-globin promoter; iii) a block in terminal erythroid differentiation. In contrast to erythroid cells lacking EKLF, $\triangle 221 \mathrm{EKLF}$ erythroid progenitors demonstrate appropriate binding of the $\triangle 221 \mathrm{EKLF}$ encoding domain to all EKLF-regulatory sequences and a chromatin architecture and histone modification pattern at erythroid-specific genes that recapitulate the events observed in wild-type EKLF erythroblasts at a similar stage of erythroid ontogeny.

Examining the role of $\triangle 221 \mathrm{EKLF}$ in megakaryopoiesis, I observed inhibition of megakaryocytic progenitor expansion in $\triangle 221 \mathrm{EKLF}$ fetal hematopoietic cell populations when compared to EKLF-null embryos. Molecular analysis of $\triangle 221 E K L F$ erythroblasts reveals i) binding of the $\Delta 221 \mathrm{EKLF}$ mutant protein to the Fli-1 promoter with inhibition of gene transcription; ii) hypoacetylation of histone $\mathrm{H} 3$ at the Fli-1 promoter; iii) recruitment of a Sin3A-containing corepressor complex to the Fli-1 promoter. Taken together, my results suggest strongly that the unique $\Delta 221 \mathrm{EKLF}$ domain is sufficient to modulate the chromatin-specific roles of EKLF at erythroid- and megakaryocytic-specific loci in definitive hematopoietic cells in vivo. 


\section{TABLE OF CONTENTS}

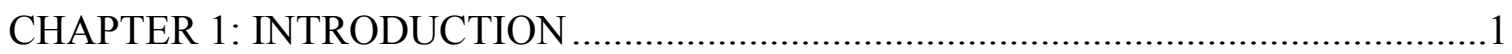

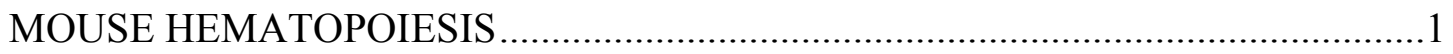

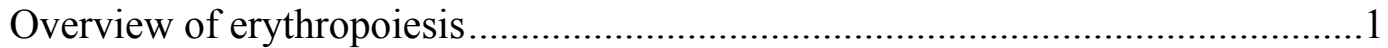

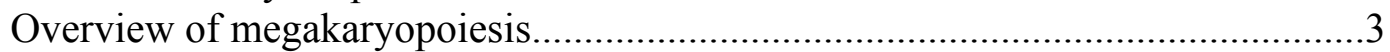

Transcription factors in erythropoiesis and megakaryopoiesis..............................3

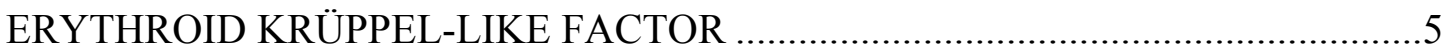

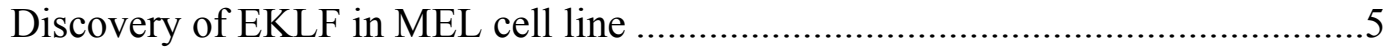

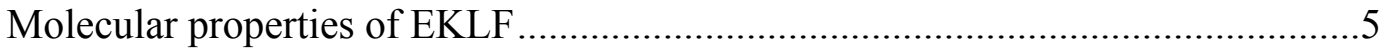

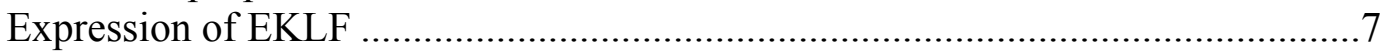

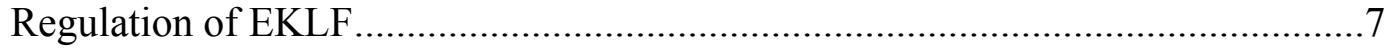

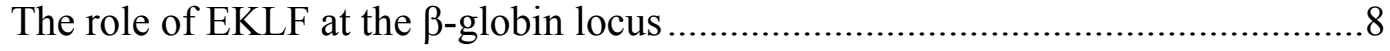

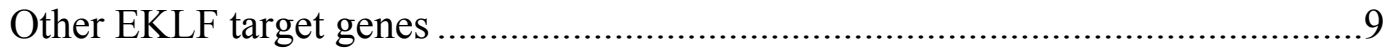

EKLF possesses distinct and separable chromatin remodeling and

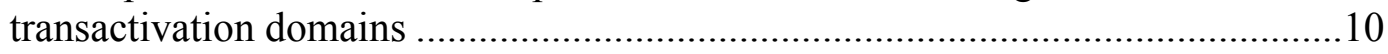

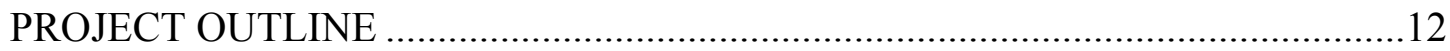

CHAPTER 2: GENERATION OF A KNOCK-IN ALLELE OF EKLF UTLIZING

RECOMBINEERING TECHNOLOGY ……………..........................................14

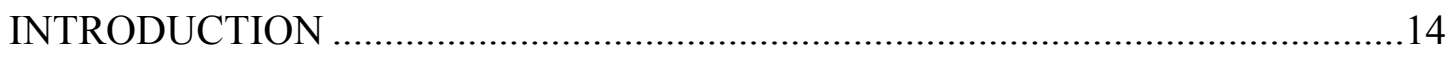

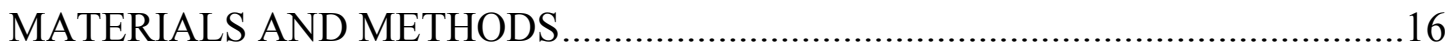

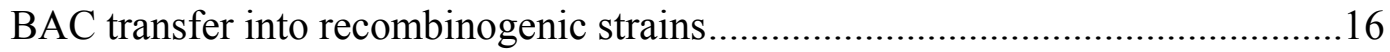

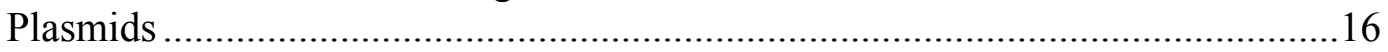

Recombineering ............................................................................................

Excision of the NEO cassette in bacteria ...........................................................17

Gene targeting in ES cells and generation of $\triangle 221$ EKLF mice............................18

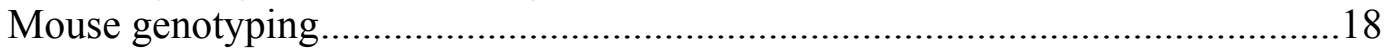

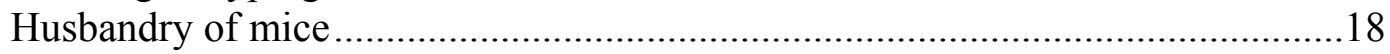

Analysis of peripheral blood counts ................................................................19

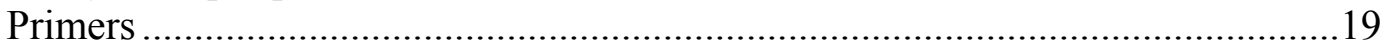

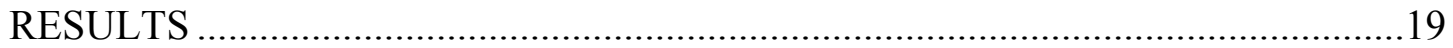

Generation and characterization of targeting vectors ............................................19

Targeting of the murine EKLF genomic locus ....................................................28

$\Delta 221 \mathrm{EKLF}$ heterozygous mice are phenotypically normal ................................28

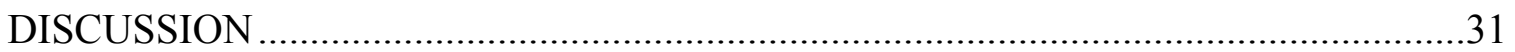




\section{CHAPTER 3: CHARACTERIZATION OF THE MOLECULAR

INTRODUCTION

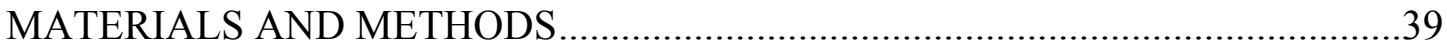

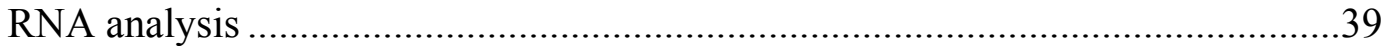

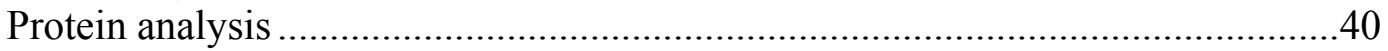

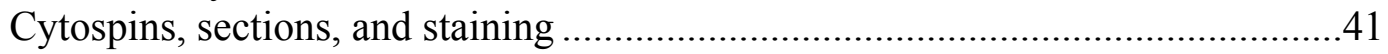

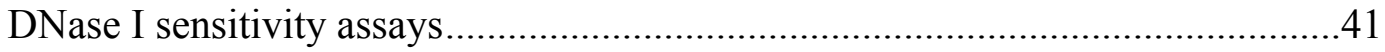

Chromatin immunoprecipitation assays........................................................42

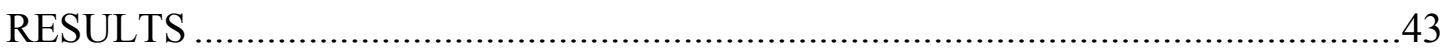

$\Delta 221 \mathrm{EKLF}$ homozygous animals die in utero and exhibit ineffective

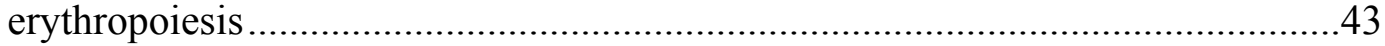

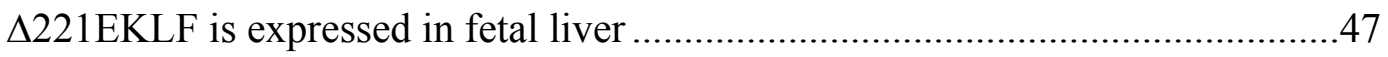

Expression of $\triangle 221 \mathrm{EKLF}$ is sufficient for $\beta$-globin promoter remodeling ...........52

Expression of $\triangle 221 \mathrm{EKLF}$ induces histone modifications at the $\beta$-globin

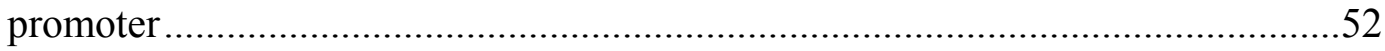

$\triangle 221 E K L F$ binds to erythroid-specific gene loci ...........................................56

Expression of $\triangle 221 \mathrm{EKLF}$ is insufficient for high-level $\beta$-globin transcription ....56

Differential binding of factors at the $\beta$-globin LCR and promoter......................56

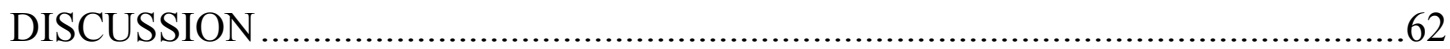

Expression of $\triangle 221 \mathrm{EKLF}$ is not sufficient to rescue the EKLF-null phenotype...62 Expression of $\triangle 221 \mathrm{EKLF}$ is sufficient for nucleosomal remodeling and histone modifications in vivo.

Nucleosomal remodeling and histone modification are not sufficient for recruitment of erythroid-specific transcription factors to the $\beta$-globin promoter

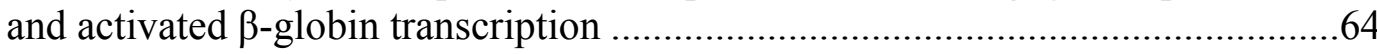

A model for $\triangle 221 \mathrm{EKLF}$ in $\beta$-globin promoter remodeling .............................65

\section{CHAPTER 4: EXPRESSION OF $\triangle 221 E K L F$ RESULTS IN ALTERED}

ERYTHROID MATURATION AND BLOCKED MEGAKARYOCYTIC

EXPANSION

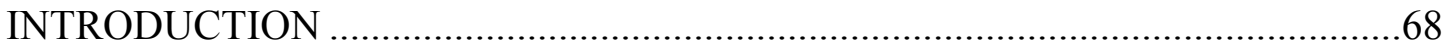

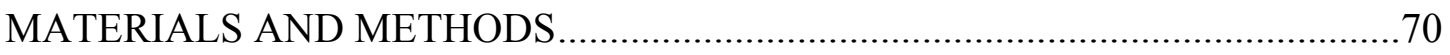

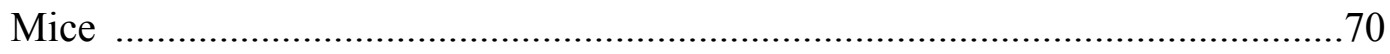

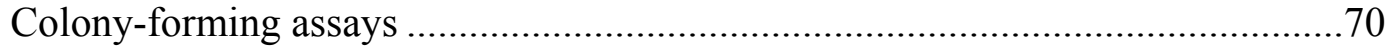

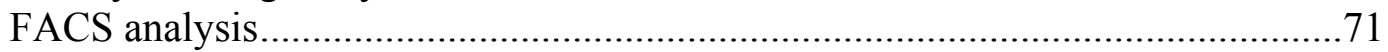

Globin gene expression by real time RT-PCR ................................................. 71

Chromatin immunoprecipitation assays........................................................ 
RESULTS

Expression of $\triangle 221 \mathrm{EKLF}$ alters erythroid differentiation and inhibits expansion of megakaryocytic progenitors

$\Delta 221 \mathrm{EKLF}$ alters expression of non- $\beta$-globin-like erythroid-specific genes .........74

A role for $\triangle 221 \mathrm{EKLF}$ in megakaryopoiesis and regulation of Fli- 1 ....

A repressor complex is recruited to the Fli-1 promoter in $\triangle 221 \mathrm{EKLF}$ cells ..........80

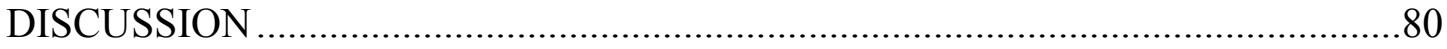

Failure of erythroid differentiation in $\triangle 221 \mathrm{EKLF}$-expressing cells ......................80

$\triangle 221$ EKLF regulates Fli-1 gene and inhibits expansion of megakaryocytic

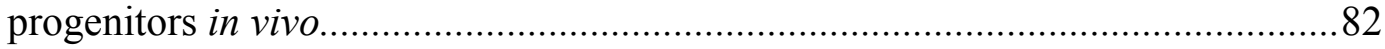

$\Delta 221$ EKLF uniquely inhibits Fli-1 by recruitment of a Sin3A-containing corepressor complex

CHAPTER 5: SUMMARY AND FUTURE DIRECTIONS

$\triangle 221 E K L F$ mice are an excellent model to study separable chromatin remodeling and $\beta$-globin gene transcription in vivo. . .85

What is the role of $\triangle 221 \mathrm{EKLF}$ in erythroid-specific transcription factories?

Is $\triangle 221 \mathrm{EKLF}$ sufficient for formation of the Active Chromatin $\mathrm{Hub}(\mathrm{ACH})$ ?

Does $\triangle 221$ EKLF regulate other megakaryocytic genes in vivo?

LIST OF REFERENCES

APPENDIX: SUPPORTING FIGURES AND TABLES

VITA 


\section{LIST OF TABLES}

Table 2.1 Predicted fragment size with restriction enzyme digestion .....................27

Table 2.2 Genotyping of adult animals from heterozygous matings .......................32

Table 2.3 Complete blood count analysis of $\triangle 221$ EKLF heterozygous

adult mice

Table 3.1 Genotyping of embryos at different stages of gestation from heterozygous matings.

Table 3.2 Quantitative analysis of benzidine-positive E14.5 fetal liver erythroblasts .46

Table A.1 Putative EKLF-dependent genes.......................................................... 105

Table A.2 Predicted fragment size with restriction enzyme digestion .....................108

Table A.3 Buffer composition used in ChIP analysis ........................................110

Table A.4 Primers used for ChIP analysis ...................................................... 111

Table A.5 Primers used for real time RT-PCR analysis ......................................112 


\section{LIST OF FIGURES}

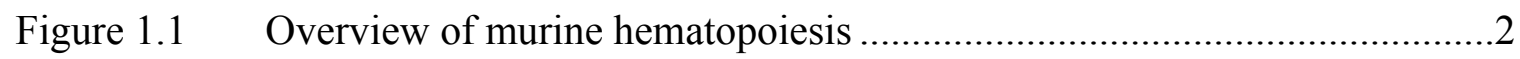

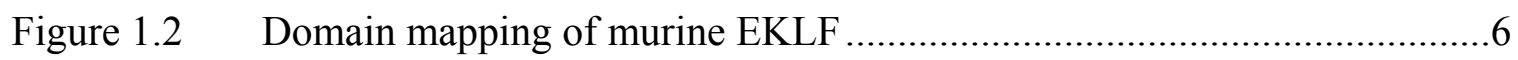

Figure 1.3 Separable chromatin remodeling and transactivation domains of EKLF

Figure 2.1 Overview of the recombineering method used to generate vectors for targeting in ES cell ...........................................................................20

Figure 2.2 Mapping of the gap-repaired plasmid ....................................................22

Figure 2.3 Analysis of $\triangle 221 \mathrm{EKLF}$ mini-targeting vector .......................................24

Figure 2.4 Verification of the final targeting vector using restriction enzyme

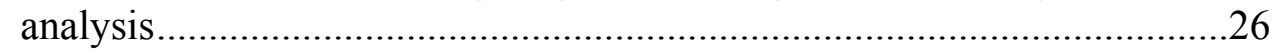

Figure 2.5 Targeting of cDNA encoding HA- $\Delta 221 \mathrm{EKLF}$ into the endogenous

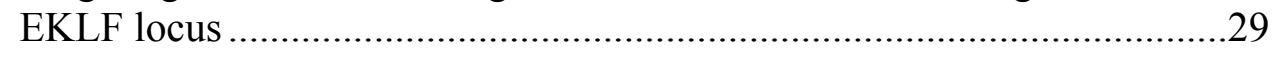

Figure 2.6 Identification of heterozygous mice expressing HA- $\triangle 221 \mathrm{EKLF} . . . . . . . . . . . . .30$

Figure 2.7 Normal circulating erythrocytes in $\triangle 221$ EKLF heterozygous adult animals

Figure $3.1 \quad \Delta 221 \mathrm{EKLF}$ embryos are anemic

Figure 3.2 Defects in cell shape and content in $\triangle 221 \mathrm{EKLF}$ fetal liver erythroblasts... .46

Figure 3.3 Accumulation of hemosiderin in fetal liver of mice expressing $\triangle 221 \mathrm{EKLF}$

Figure 3.4 Expression of $\triangle 221 \mathrm{EKLF}$ in fetal liver

Figure 3.5 Localization of $\triangle 221 \mathrm{EKLF}$ knock-in protein ...

Figure 3.6 DNase I sensitivity at the $\beta$-globin promoter in $\triangle 221 \mathrm{EKLF}$ fetal liver erythroblast

Figure 3.7 Global acetylation of histone $\mathrm{H} 3$ across the $\beta$-globin locus .54

Figure 3.8 Trimethylation pattern at lysine 4 of histone $\mathrm{H} 3$ .55 
Figure 3.9 Differential binding of $\triangle 221 \mathrm{EKLF}$ to erythroid-specific gene loci

Figure 3.10 Lack of $\beta$-globin transcripts in $\triangle 221 \mathrm{EKLF}$ fetal liver erythroblasts in vivo

Figure 3.11 Lack of RNA polymerase at the $\beta$-globin promoter in $\triangle 221 \mathrm{EKLF}$ expressing cells

Figure 3.12 Binding of erythroid-specific transcription factors at the $\beta$-globin promoter in vivo

Figure 3.13. Models of $\beta$-globin promoter remodeling in erythroid cells .66

Figure 4.1 Potential models of EKLF-mediated repression of megakaryopoiesis

Figure 4.2 Effects of $\Delta 221 \mathrm{EKLF}$ expression on terminal erythroid differentiation and proliferation of fetal liver progenitor cells.

Figure 4.3 Altered pattern of erythroid-specific gene expression in $\triangle 221 \mathrm{EKLF}$ fetal liver erythroblasts

Figure 4.4 Blocked expansion of megakaryocytic progenitors in $\triangle 221 \mathrm{EKLF}$ mice

Figure 4.5 Differential expression of $\beta$-globin and Fli-1 gene in $\triangle 221 E K L F$ expressing cells .78

Figure 4.6 Distinct regulation of $\beta$-globin and Fli-1 promoters in $\triangle 221 E K L F$ fetal liver erythroblasts .79

Figure 4.7 Recruitment of a Sin3A-containing repressor complex.....

Figure 4.8 Modified potential models of $\triangle 221 \mathrm{EKLF}$-mediated repression of megakaryopoiesis

Figure A.1 Confirmation of the mini-targeting vectors

Figure A.2 Sequences of Southern blot probes used in screening targeted ES cells and genotyping of animals.

Figure A.3 Fetal liver hematopoietic progenitors 


\section{LIST OF ABBREVIATIONS}

$\mathrm{ACH}$

AHSP

ALAD

ALAS2

AMP

APC

BAC

BFU-E

BKLF

cDNA

CFU

CFU-E

CFU-GEMM

CFU-GM

CFU-MK

ChIP

CLP

CMP

$\mathrm{COOH}$

CPOX

Da

DAPI

dsDNA

DT

EKLF

En-1, En-2

EPB4.9

E-RC1

ES

FECH

FITC

FOG1

GAL4

H\&E

HA

HCT

HGB

HS

HSC

IP

IVR

$\mathrm{kDa}$
Active Chromatin Hub

Alpha Hemoglobin Stabilizing Protein

Aminolevulinate Dehydratase

Aminolevulinic Acid Synthase 2

Ampicillin

Allophycocyanin

Bacteria Artificial Chromosome

Burst Forming Unit-Erythroid

Basic Krüppel-like Factor

Complementary DNA

Colony Forming Unit

CFU-Erythroid

CFU-Granulocyte-Erythroid-Monocyte-

Megakaryocyte

CFU-Granulocyte-Macrophage

CFU-Megakaryocyte

Chromatin Immunoprecipitation

Common Lymphoid Progenitor

Common Myeloid Progenitor

Carboxyl

Coproporphyrinogen Oxidase

Dalton

4',6-Diamidino-2-Phenylindole

Double-stranded DNA

Diptheria Toxin

Erythroid Krüppel-like Factor

Engrailed

Erythroid Protein Band 4.9

EKLF Coactivator-Remodeling Complex 1

Embryonic Stem

Ferrochelatase

Fluorescein Isothiocyanate

Friend of GATA-1

Galactose 4

Hematoxylin and Eosin

Hemagglutinin

Hematocrit

Hemoglobin

Hypersensitive Site

Hematopoietic Stem Cell

Immunoprecipitation

Intervening region

KiloDalton 
$\mathrm{KO}$

LB

LCR

LMO2

Lys

MEL

MEP

mRNA

Myf5

NEO

NF-E2

$\mathrm{NH} 2$

NLS

PAC

PBGD

PCR

PE

PGK

PLT

PMSF

PolII

PPOX

$\mathrm{RBC}$

RT-PCR

SCL

SDS-PAGE

SEM

shRNA

TF

TK

UROD

WBC

WT
Knock-out

Luria Broth

Locus Control Region

LIM Domain Only 2

Lysine

Mouse Erythroleukemic

Megakaryocyte-Erythrocyte Progenitor

Messenger RNA

Myogenic factor 5

Neomycin

Nuclear Factor - Erythroid 2

Amino

Nuclear Localizing Signal

P1 Artificial Chromosome

Porphobilinogen Deaminase

Polymerase Chain Reaction

Phycoerythrin

Phosphoglycerate Kinase

Platelet Count

Phenylmethylsulphonyl Fluoride

Polymerase II

Protoporphyrinogen Oxidase

Red Blood Count

Reverse Transcriptase Polymerase Chain Reaction

Stem Cell Leukemia

Sodium Dodecyl Sulfate Polyacrylamide Gel

Electrophoresis

Standard Error Mean

Short Hairpin RNA

Transcription Factor

Thymidine Kinase

Uroporphyrinogen Decarboxylase

White Blood Count

Wild Type 


\section{CHAPTER 1: INTRODUCTION}

\section{MOUSE HEMATOPOIESIS}

Hematopoiesis is the formation and development of blood cells involving the differentiation of a multipotent progenitor, the hematopoietic stem cell (HSC), and its progeny into all blood cell lineages (Figure 1.1) (Orkin \& Zon, 2008). This complex continuous process requires a broad spectrum of lineage-specific transcription factors (TFs), such as SCL, LMO2, c-myb, PU.1, EKLF, GATA-1, NF-E2, and Fli-1 (Shivdasani \& Orkin, 1996; Perry \& Soreq, 2002; Orkin \& Zon, 2008). Genetic studies involving the disruption or over-expression of these genes have facilitated our understanding of the transcriptional regulation of hematopoiesis (Perry \& Soreq, 2002). Many of these TFs are beyond the scope of my dissertation and will not be addressed. In the context of this

dissertation, I will explore the role of erythroid-Krüppel-like factor, or EKLF, in $\beta$-globin gene regulation during erythroid differentiation (Chapter 3). Furthermore, I will demonstrate that the chromatin remodeling encoding domain of EKLF is sufficient to repress expansion of megakaryocytic progenitors observed with complete loss of EKLF (Chapter 4).

\section{Overview of erythropoiesis}

Erythropoiesis is the development of mature red blood cells from hematopoietic stem cells (Orkin \& Zon, 2008). In mammals, this process occurs in the bone marrow and is characterized by three stages (Palis, 2009). The first stage involves production of lineage-committed progenitors. The earliest recognizable erythroid-specific progenitor is the burst-forming unit erythroid (BFU-E). The BFU-E generates more mature erythroid-committed progenitors termed colony-forming units erythroid (CFU-E) (Socolovsky et al., 1998). BFU-E and CFU-E can be detected in vitro using colonyforming assays (Ogawa et al., 2002). The second stage of erythroid differentiation consists of the progression of erythroid precursors from early proerythroblasts into orthochromatic erythroblasts. This stage of erythroid differentiation is characterized by the progressive accumulation of hemoglobin, expansion of erythroblasts, and progressive nuclear pyknosis and ultimately loss of the nucleus. The final stage of erythropoiesis involves maturation of the young red blood cells (reticulocytes) into mature circulating red cells (Palis, 2009).

Erythropoiesis occurs in distinct phases and anatomic sites during murine development (Dzierzak \& Medvinsky, 1995; Zon, 1995; Shivdasani \& Orkin, 1996; Palis, 2008). The first phase can be identified in the yolk sac at embryonic day 7.5 (E7.5) in mice and is referred to as primitive or embryonic erythropoiesis. Primitive erythrocytes are large cells that retain their nuclei and produce embryonic globin chains $(\zeta, \varepsilon / \beta h 1)$. The adult $\beta$-globin genes are silent. By E11, definitive erythropoiesis is initiated in the fetal liver. At this stage, the adult globin $(\alpha, \beta$ maj $/ \beta$ min $)$ genes are expressed and the embryonic globin genes are silenced. The molecular mechanisms regulating the 


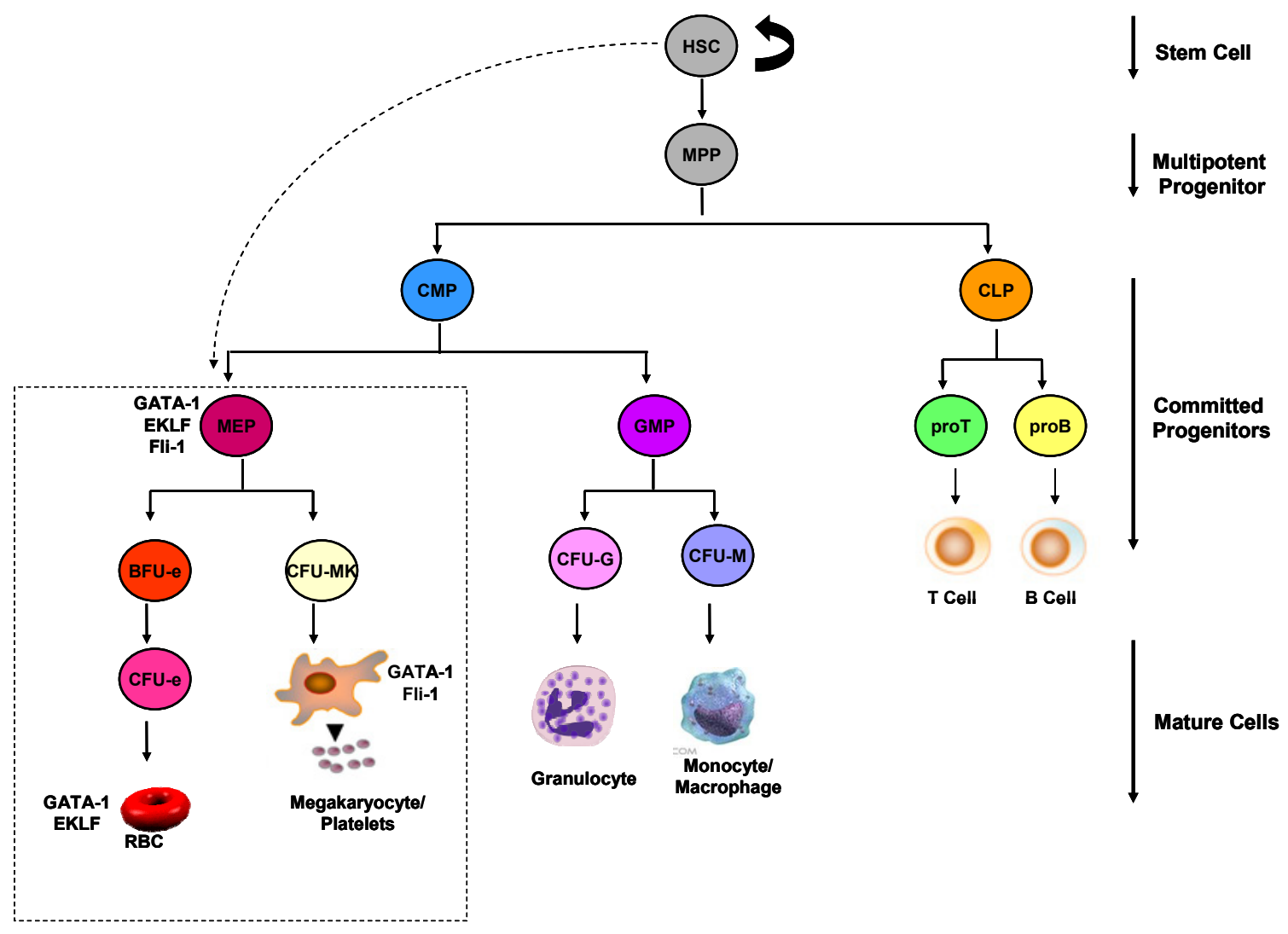

Figure 1.1. Overview of murine hematopoiesis. All blood cell types arise from the self-renewing hematopoietic stem cell (HSC) that differentiates into committed progenitor cells to produce mature blood cells. The erythroid and megakaryocytic lineage is thought to have come from a common bipotent progenitor (megakaryocyticerythroid progenitor (MEP) (dotted box). The MEP population is thought to be formed from common myeloid progenitor (CMP); recent evidence proposes a direct pathway from HSCs to MEP (dotted line). Key transcription factors regulating this process are listed by the cell type in which they are expressed. Abbreviations: MPP, multipotent progenitor; CLP, common lymphoid progenitor; GMP, granulocyte and macrophage progenitor; $\mathrm{CFU}-\mathrm{G}$, colony-forming unit granulocyte; CFU-M, colony-forming unit monocyte/macrophage; BFU-E, blast-forming unit erythrocyte; CFU-E, colony-forming unit erythrocyte; MK-P, megakaryocyte progenitor. 
switching of globin gene expression has been the intense focus of many laboratories. They have provided a critical foundation for our understanding of the molecular mechanism underpinning erythropoiesis.

\section{Overview of megakaryopoiesis}

The erythroid and megakaryocyte lineages are thought to be derived from a common precursor, the bipotent megakaryocyte-erythroid progenitor (MEP) (Debili et al., 1996). In the most established pathway, HSCs generate common myeloid progenitor (CMP) cells from which the MEP is formed (Akashi et al., 2000). However, it has been recently proposed that HSCs can give rise to MEP population without an intermediate progenitor (Adolfsson, 2005).

Megakaryopoiesis is the process by which HSCs differentiate into mature megakaryocytes through a series of differentiated progenitors. Megakaryocytic progenitors are detected in the yolk sac and fetal liver at approximately E7.5-10.5 and E11.5, respectively (Tober et al., 2007). The earliest committed MK progenitor is the burst-forming unit megakaryocyte (BFU-MK) that gives rise to the more mature colonyforming unit megakaryocyte (CFU-MK) (Briddellet al., 1989). The CFU-MK then gives rise to megakaryoblasts that in turn differentiate into mature megakaryocytes.

\section{Transcription factors in erythropoiesis and megakaryopoiesis}

The precursor cells express many common hematopoietic transcription factors that are essential to both erythropoiesis and megakaryopoiesis, including GATA-1 (Pevny et al., 1995; Shivdasani et al., 1997), FOG1 (Tsang et al., 1997), and SCL (Hall et al., 2003). The MEP lineage differentiation is regulated in part by the differential expression and combinatorial action of these transcription factors.

\section{GATA-1}

GATA-1, the founding member of the GATA family of zinc finger proteins, is an erythroid transcription factor that binds to the DNA sequences WGATAR found at the cis-regulatory sequences of nearly all erythroid genes, including the $\beta$-globin gene (Martin \& Orkin, 1990; Weiss \& Orkin, 1995). GATA-1 is also expressed and has defined functional activities in megakaryocytes, eosinophils, and mast cells (Zon et al., 1993). Hemizygous deletion of GATA-1, located on the X-chromosome, leads to loss of erythropoiesis and embryonic lethality by E11.5 (Fujiwara et al., 1996). GATA-1 null mice also display a block in megakaryocyte development. However, GATA-1 null ES cells can develop into other hematopoietic lineages (Kitajima et al., 2006). Similarly, forced expression of GATA-1 in an early myeloid cell line promotes megakaryocytic differentiation, suggesting that GATA-1 has a key role in lineage determination (Shivdasani \& Orkin, 1996; Shivdasani et al., 1997). Studies to identify proteins that 
bind to GATA-1 led to the discovery of Friend of GATA (FOG-1), a nuclear zinc finger protein that binds the amino zinc finger of GATA-1 (Tsang et al., 1997). Expression of FOG-1 is similar to that of GATA-1. Like mice lacking GATA-1, FOG-1 null mice do not form megakaryocytic progenitors and are embryonic lethal at E11.5 (Tsang et al., 1998).

$N F-E 2$

NF-E2 is a hematopoietic transcription factor belonging to the basic-leucine zipper family of dimeric proteins consisting of a ubiquitously expressed $18 \mathrm{kDa}$ subunit and a tissue-specific $45 \mathrm{kDa}$ subunit (Chan et al., 1993). Initial studies in cell lines provided evidence that NF-E2 is essential for $\beta$-globin gene expression (Lu et al., 1994). Surprisingly, mice lacking the $45 \mathrm{kD}$ hematopoietic-restricted subunit develop only a mild erythroid phenotype, but exhibit severe thrombocytopenia with bone marrow showing excessive immature and dysplastic megakaryocytes (Shivdasani \& Orkin, 1995). The subtle effects on erythroid maturation are presumably due to functional redundancy between NF-E2 and other basic leucine zipper family proteins (Sawado et al., 2001). By contrast, the molecular basis for the severe defects in megakaryocyte differentiation and platelet release remains to be elucidated.

\section{Fli-1}

Fli-1 is a member of the Ets family of transcription factors (Watson et al., 1992) containing a conserved winged helix-loop-helix DNA binding (ETS) domain which has critical functions in development and oncogenesis (Jackers et al., 2004). Fli-1 is preferentially expressed in cells of the hematopoietic lineages and vascular endothelium. Fli-1 has been shown to transcriptionally activate many genes, including those involved in megakaryopoiesis. In undifferentiated hematopoietic cell lines, overexpression of Fli1 can induce megakaryocytic features and inhibit erythroid differentiation (Pereira et al., 1999; Athanasiou et al., 2000). Moreover, Fli-1 knock-out mice either have abnormal megakaryocytes with associated thrombocytopenia (Hart et al., 2000) or fail to develop recognizable megakaryocytes (Kawada et al., 2001). These observations are consistent with the idea that Fli-1 is a key factor in the lineage fate decision leading to the production of megakaryocytes.

Interestingly, these factors are interconnected with my gene of interest, EKLF (reviewed in a separate section below). EKLF, GATA-1, and Fli-1 are all expressed in the MEP (Orkin \& Zon, 2008). GATA-1 transcriptionally activates EKLF (Crossley et al., 1994), while Fli-1 and EKLF interactions have been noted (Starck et al., 2003). Moreover, recent data including that from our laboratory suggest that EKLF represses Fli-1 expression (J.M.C unpublished data; Frontelo et al., 2007). Similarly, both EKLF and NF-E2 are essential for high level $\beta$-globin gene transcription (Asano \& Stamatoyannopoulos, 1998; Sawado et al., 2001). However, the relationship between EKLF and NF-E2 in megakaryopoiesis is relatively unknown. Although associations 
between these transcription factors and many others are recognized in erythroid and megakaryocytic differentiation, the mechanisms underlying these relationships have yet to be resolved.

\section{ERYTHROID KRÜPPEL-LIKE FACTOR}

\section{Discovery of EKLF in MEL cell line}

The murine erythroleukemia (MEL) cell line has proven a popular and highly important murine model system to study erythroid-specific gene expression. These erythroid progenitor cells, immortalized by infection with Friend virus (Friend, 1957; Antoniou, 1991), are arrested at the proerythroblast stage of development. This cell can be maintained in tissue culture indefinitely (Friend, 1957; Antoniou, 1991). However, upon treatment with various chemical agents, MEL cells can be induced to undergo erythroid differentiation with the induction of globin and other erythroid genes involved in the terminal erythroid differentiation program (Marks \& Rifkind, 1988; Radhika et al., 1995). These cells have also proven to be useful to study megakaryocytic differentiation (Bouilloux et al., 2008).

This transformed cell line is an ideal model to identify novel genes which may play a role in erythropoiesis. Using subtractive hybridization and enriching for genes expressed in a MEL cell line, but not in a murine monocyte-macrophage cell line, a novel erythroid cell-specific zinc finger protein was isolated (Miller \& Bieker, 1993). Close inspection of the zinc finger region of this factor revealed that it is similar to the Drosophila pattern-determining gap gene Krüppel. Therefore, the protein was named EKLF/KLF1, for erythroid Krüppel-like factor (Miller \& Bieker, 1993).

\section{Molecular properties of EKLF}

EKLF maps to a region on mouse chromosome 8 (Jenkins et al., 1998) and human chromosome 19 (Bieker, 1996; van Ree et al., 1997). The EKLF gene spans $\sim 6.5 \mathrm{~kb}$ and contains 3 exons. There are two major transcripts produced, the results of alternative transcriptional start sites at nucleotides 41 and 55. This is not uncommon as multiple transcription start sites have been observed for other tissue-specific genes, namely the heterogeneous 5' ends of both c-myb and GATA-1 (Bender \& Kuehl, 1986; Tsai et al., 1989). Sequence analysis reveals open reading frames beginning at the in-frame methionines 1 and 19. Because methionine 1 does not match the Kozak consensus sequence, translation of the major product starts from methionine 19, encoding an EKLF protein of 358 amino acids (37,755 Da) (Miller \& Bieker, 1993). The protein has a carboxy-terminal DNA-binding domain consisting of three $\mathrm{C} 2 \mathrm{H} 2$ zinc fingers and a proline-rich amino domain that has structural similarities to other transactivation domains (Figure 1.2). 


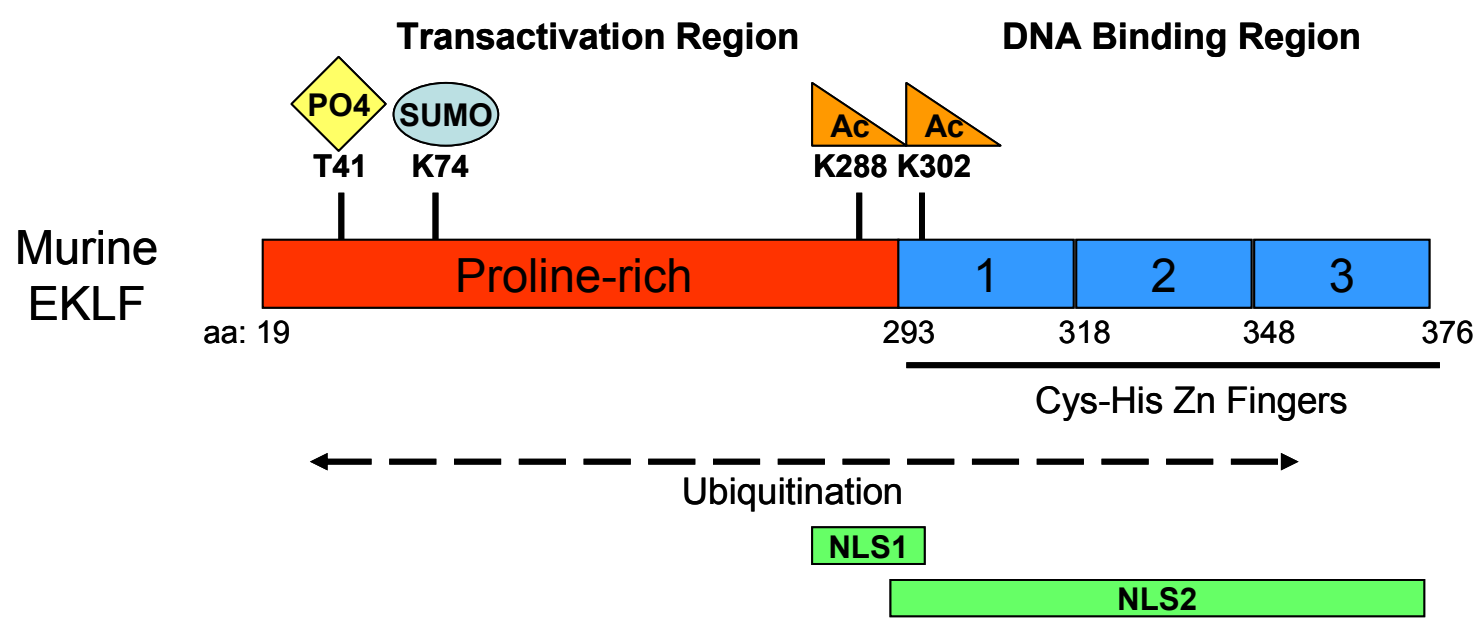

Figure 1.2. Domain mapping of murine EKLF. This schematic diagram summarizes the EKLF protein showing its transactivation (amino terminal) and DNA binding (carboxyl terminal) regions. EKLF encodes a protein of 376 amino acids characterized by a proline-rich transactivation domain. The DNA binding domain contains three $\mathrm{C} 2 \mathrm{H} 2$ zinc fingers. Also noted in the diagram are the locations of important phosphorylation (T41), sumoylation (K74), acetylation (K288 and K302), and ubiquitination (throughout protein) sites, and two NLS discussed in the text. 
EKLF/KLF1, the founding member of a 15 gene clade, interacts with the DNA consensus sequence CCNCNCCCN found at many promoters (Miller \& Bieker, 1993). EKLF has two nuclear localization signals (NLSs) corresponding to a region adjacent to the zinc finger DNA binding domain within a stretch of highly basic amino acids 275-296 (Quadrini \& Bieker, 2002) and another more efficient signal within the zinc finger domain itself encompassing amino acids 293-376 (Pandya \& Townes, 2002; Quadrini \& Bieker, 2002). Interestingly, each zinc finger is important for the overall function of the NLSs, and a complete zinc finger structure is necessary for efficient nuclear localization.

\section{Expression of EKLF}

Both human and murine EKLF have been cloned and show a high degree of homology with $>90 \%$ similarity in the zinc fingers and approximately $70 \%$ within the proline-rich amino domain (Bieker, 1996; van Ree et al., 1997). Expression of both factors is restricted to the erythroid lineage with high levels in murine and human definitive hematopoietic progenitors (Miller \& Bieker, 1993; Bieker, 1996; van Ree et al., 1997). During in vitro hematopoietic cell differentiation of murine ES cells, EKLF is expressed in the CMP and MEP population (Frontelo et al., 2007). EKLF expression is absent in lymphoid cell lines (Miller \& Bieker, 1993) and the CLP and their progeny (Frontelo et al., 2007).

During murine ontogeny, EKLF is expressed early and at different anatomical sites (Southwood et al., 1996). EKLF mRNA is detected first at the neural plate stage (at E7.5) within the blood islands in the yolk sac. EKLF is then expressed within the hepatic tissue beginning with the earliest stage of hepatic formation at E9 and continuing until E14.5 when the liver becomes the only source of EKLF. Concomitantly with EKLF mRNA, EKLF protein is also expressed in primitive cells and in the fetal liver. In the adult animal, EKLF expression is strictly localized to the red pulp of the spleen.

\section{Regulation of EKLF}

EKLF is a stage-and lineage-specific transcription factor, its expression requiring tight regulation. EKLF expression is induced by Bmp4/Smad signaling and GATA-1 (Adelmann et al., 2002; Lohmann \& Bieker, 2008). However, regulation of EKLF activity is achieved in part by post-translational modifications of the EKLF protein (see Figure 1.2). First, EKLF is a phosphoprotein whose transcriptional activity is dependent on the phosphorylation status at threonine 41 (T41) (Ouyang et al., 1998). On the other hand, sumoylation of EKLF at lysine 74 (K74) promotes transcriptional repression of megakaryopoiesis (Siatecka et al., 2007). Similarly, EKLF is acetylated by CBP/p300 (Zhang \& Bieker, 1998; Zhang et al., 2001). This modification increases EKLF's affinity for the SWI/SNF components of chromatin remodeling complexes which has been postulated to maintain chromatin in an open configuration (Armstrong et al., 1998; Kadam et al., 2000; Zhang et al., 2001). On the other hand, EKLF acetylation can also result in interaction with co-repressors Sin $3 \mathrm{~A}$ and recruitment of histone deacetylases 
(HDACs) to promoters to inhibit gene transcription (Chen \& Bieker, 1996; Chen \& Bieker, 2004). Finally, EKLF can be ubiquitinated and degraded through the ubiquitinmediated proteosome pathway (Quadrini \& Bieker, 2006).

\section{The role of EKLF at the $\beta$-globin locus}

Disruption of the EKLF gene by homologous recombination has demonstrated its non-redundant role in erythropoiesis. EKLF-null embryos die of a lethal anemia by embryonic day 16 (E16), as definitive erythroid cells fail to produce $\beta$-globin transcripts in vivo (Nuez et al., 1995; Perkins et al., 1995), consistent with the idea that EKLF is essential for $\beta$-globin gene transcription. However, it is now recognized that EKLF also regulates expression of multiple erythroid-specific genes, including cytoskeletal proteins (Nilson et al., 2006) and alpha hemoglobin stabilizing protein (AHSP) (Pilon et al., 2006). Moreover, analysis of EKLF-null embryos that are transgenic for the human $\beta$-globin locus confirmed the necessity of EKLF for human $\beta$-globin gene transcription (Perkins et al., 1996; Wijgerde et al., 1996). Studies in EKLF-null animals have delineated three mechanisms of action for EKLF in regulating $\beta$-globin gene transcription: i) chromatin remodeling, ii) modulation of transactivation, and iii) stabilization of the locus control region (LCR)/ $\beta$-globin promoter interaction.

\section{EKLF alters chromatin structure at the $\beta$-globin promoter}

Local chromatin structure plays a critical role in regulating gene expression. Transcriptionally active genes are typically found in regions of open chromatin structure characterized by DNase I-sensitivity and histone hyperacetylation whereas inactive genes are packaged in a highly condensed chromatin configuration that is typically DNase Iinsensitive and under-acetylated (Harju et al., 2002). Chromatin structure may be altered by changing the organization of the nucleosome(s) at the gene promoter or by modifying the histones (Harju et al., 2002). Thus, chromatin remodeling is an essential event for the initiation of gene transcription in vivo.

EKLF is a key player in activating $\beta$-globin gene transcription. Not only has it been postulated to be necessary for transactivation but it was the first factor implicated in erythroid-specific promoter remodeling of the $\beta$-globin promoter. Analysis of EKLF-null embryos revealed a specific loss of a developmentally specific DNase I hypersensitive site in the proximal $\beta$-globin promoter (Wijgerde et al., 1996). Since the degree of DNase I hypersensitivity of a given locus correlates with nucleosomal remodeling (Steger \& Workman, 1996; Pazin et al., 1997), these findings strongly suggest that EKLF is required for chromatin reorganization at the $\beta$-globin promoter in definitive erythroid cells. A loss of DNase I hypersensitivity was also observed in hypersensitive site 3 (HS3) of the LCR, but to a lesser extent. Furthermore, utilizing chromatin immunoprecipitation (ChIP) analysis, we observed increased acetylation of histone $\mathrm{H} 3$ at the $\beta$-globin promoter after induction of EKLF in an EKLF-inducible erythroid cell system. This change correlates with activation of $\beta$-globin gene transcription (J.M.C 
unpublished data). These results confirmed the role of EKLF as a chromatin modulator and transcriptional activator of the $\beta$-globin gene. However, the specific domains that fulfill this role in vivo and the molecular mechanisms responsible for chromatin modification remain to be elucidated.

\section{EKLF-mediated transactivation}

EKLF is an erythroid-specific transcription factor containing a carboxy-terminal zinc finger DNA-binding domain and a proline rich amino terminal domain. Initial structure-function studies, utilizing chimeric proteins consisting of the DNA-binding domain of the yeast factor GAL4 fused in frame to various EKLF sequences, demonstrate that the amino terminal region could be divided into two domains with opposing functions (Chen \& Bieker, 1996). In these assays, the first 104 amino acids activate GAL4-dependent transcription, whereas an internal domain (aa 196-291) mediated transcriptional repression. In contrast to these studies that utilize heterologous promoters, our laboratory has shown that an internal domain of EKLF is sufficient for activation of the endogenous $\beta$-globin promoter (Brown et al., 2002). However, it remains unclear what functions of EKLF are required for $\beta$-globin gene transactivation in vivo.

\section{The role of EKLF in $L C R / \beta$-globin promoter interaction}

Regulation of the $\beta$-globin locus is believed to occur in part by competition of each globin gene promoter for direct interaction with the LCR with the intervening region looping out. Direct support for EKLF being involved in stabilizing the LCR/ $\beta$-globin promoter interaction was provided by utilizing chromosome conformation capture $(3 \mathrm{C})$ technology (Dekker et al., 2002) to study the spatial organization of the $\beta$-globin locus. In erythroid cells, the hypersensitive sites of the LCR are in close physical proximity to the active globin genes with the intervening DNA sequence containing the inactive globin genes looped out forming the Active Chromatin $\mathrm{Hub}(\mathrm{ACH})$, a nuclear compartment dedicated to RNA polymerase II mediated transcription (Palstra et al., 2003). Subsequent studies in EKLF-null fetal livers demonstrated that EKLF is required for ACH formation and active $\beta$-globin gene transcription (Drissen et al., 2004). Despite the significant insights into the binding sites, and the effects of wild-type EKLF action, it remains unclear whether differing domains of EKLF are required for alteration of the $\beta$-promoter and LCR architecture in vivo.

\section{Other EKLF target genes}

Early observations alluded to a broader role for EKLF in the regulation of genes other than $\beta$-globin. First, the EKLF-null phenotype is more severe than that observed in a murine model of $\beta$-thalassemia in which the adult $\beta$-globin genes are deleted by homologous recombination (Ciavatta et al., 1995). Furthermore, enforced expression of an EKLF-independent globin transgene fails to rescue the lethal phenotype (Perkins et al., 
2000). Gene profiling studies by our laboratory and many others provide a list of potential target genes (see Appendix Table A.1). Subsequent studies utilizing chromatin immunoprecipitation (ChIP) analysis have confirmed dematin (Hodge et al., 2006), AHSP (Keys et al., 2007; Pilon et al., 2006), ankyrin, Band 3 (Nilson et al., 2006), and BKLF (Funnell et al., 2007) as direct EKLF target genes. Together these observations support a broader role for EKLF in the regulation of other erythroid-specific genes and provide additional evidence that defects in addition to $\beta$-globin deficiency contributes to the lethal phenotype in EKLF-null mice.

\section{EKLF possesses distinct and separable chromatin remodeling and transactivation domains}

To explore the determinants of EKLF-dependent $\beta$-globin gene activation, our laboratory utilized an erythroblast cell line lacking endogenous EKLF expression, $\mathrm{J} 2 \mathrm{e} \Delta$ eklf (Coghill et al., 2001). Briefly, this line was prepared by immortalization of fetal liver erythroblasts derived from E14.5 EKLF-null embryo by transduction with a raf/myc retrovirus. Subsequently, the cells were transduced with retroviral constructs containing EKLF cDNA fused in frame with the influenza hemagglutinin (HA) epitope at the amino terminus and the tamoxifen-binding domain of the estrogen receptor $\left(\mathrm{ER}^{\mathrm{TM}}\right)$ at the carboxyl terminus. Constructs containing full length human EKLF and a series of amino terminal mutants were studied (Figure 1.3A) (Coghill et al., 2001; Brown et al., 2002). Subsequently, these cell lines were utilized to study the distinct domains of EKLF in $\beta$ globin gene activation.

To determine the effects of the amino terminal deletions on formation of hypersensitive sites at the $\beta$-globin promoter, nuclei of induced cells for each mutant were incubated with increasing amounts of DNase I (Figure 1.3B). Contrary to an in vitro study that demonstrated the DNA binding domain of EKLF alone could induce a specific DNase I hypersensitive site of chromatinized $\beta$-globin template (Kadam et al., 2001), the hypersensitive site pattern in J2e $\Delta$ eklf cells expressing the $\Delta 253 \mathrm{EKLF}$ mutant is similar to that observed in J2e $\Delta$ eklf null cells. In contrast, cells expressing $\Delta 221 \mathrm{EKLF}$ and $\triangle 164 \mathrm{EKLF}$ show a hypersensitive site pattern similar to that observed with fulllength EKLF (flEKLF). Therefore, the sequence between aa 221-253 is required and sufficient for an "open" configuration at the endogenous $\beta$-promoter (Brown et al., 2002).

Based on prior structural analysis of EKLF, the activation domain of EKLF should reside in sequences upstream of the $\Delta 221-253$ regions. Our hypersensitive studies suggested that the transcriptional and chromatin remodeling domain activities of EKLF are separable. To test this hypothesis, transcriptional activity for each mutant was measured by RNA protection assay and correlated with its chromatin remodeling properties (Figure 1.3C). As anticipated, no significant level of the $\beta$ maj transcript was detected in $\triangle 253 E K L F$ cells. In contrast, expression of the $\triangle 221 E K L F$ polypeptide resulted in a small increase in gene transcripts. However, examination of cells expressing $\triangle 164 E K L F$ revealed levels of $\beta$ maj transcripts that paralleled flEKLF expression. Thus, 
A.

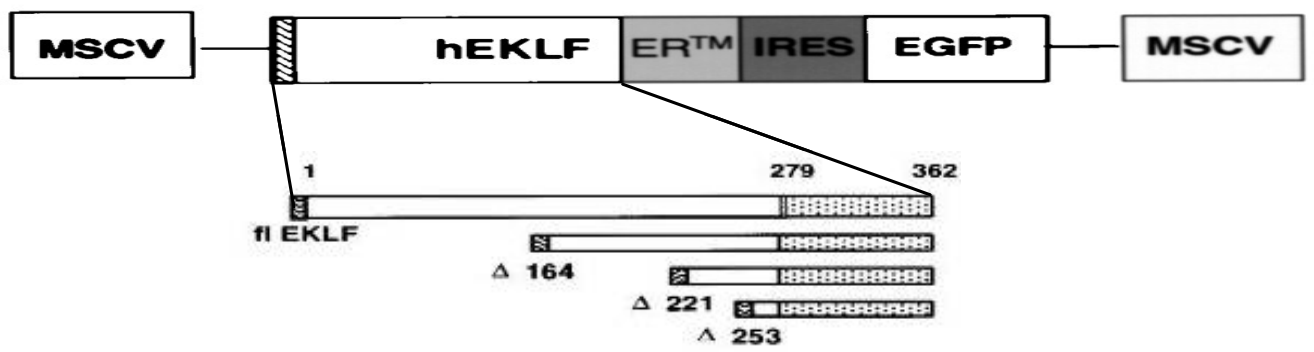

B.

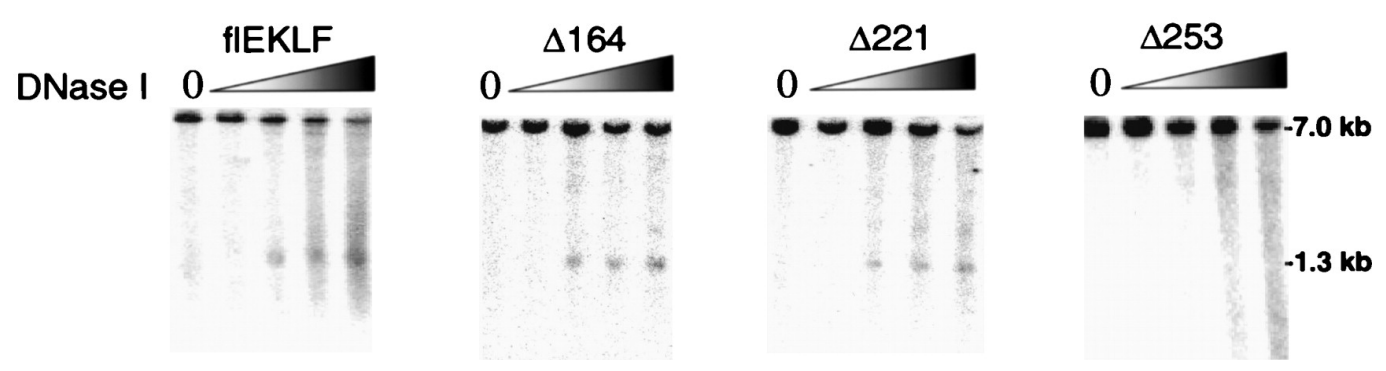

C.

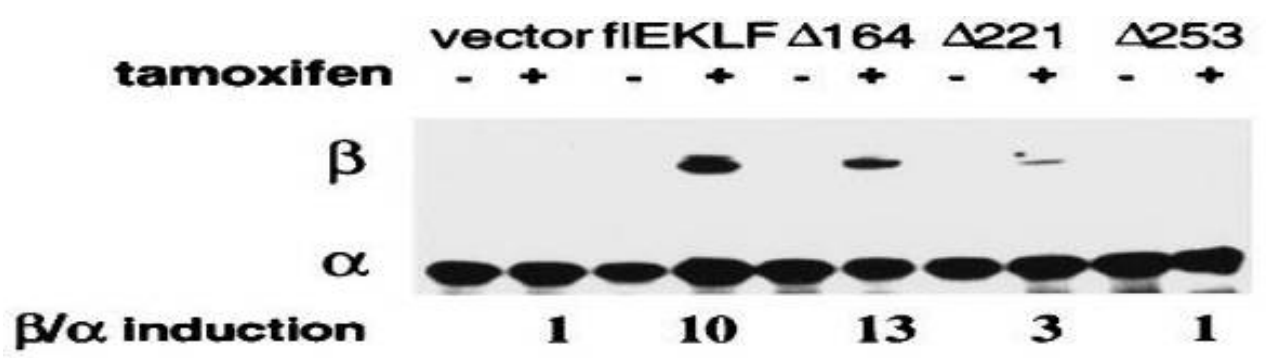

Figure 1.3. Separable chromatin remodeling and transactivation domains of EKLF. (A) Schematic diagram of flEKLF retrovirus and derivative mutants used to stably transduce J2e eklf cells. (B) The DNA binding domain $(\Delta 253)$ is required but is not sufficient for chromatin remodeling at the Bmaj globin promoter. Cells expressing each EKLF mutant were cultured for $48 \mathrm{~h}$ in the presence of tamoxifen. Nuclei were isolated and exposed to increasing concentrations of DNase I as previously described. DNA was harvested, digested with EcoRI, and probed with a Bmaj-specific probe. The DNase I concentration is 0 in the first lane of each panel and increases as shown by the shaded triangle. (C) An internal domain of EKLF $(\Delta 164)$ is sufficient to activate $\beta$-globin gene expression to wild-type levels. RNA was harvested from J2e eklf clones expressing varying EKLF mutant moieties at $48 \mathrm{~h}$ post-tamoxifen induction RPA was performed utilizing Bmaj and riboprobes. The numbers underneath the panel represent the mean fold induction of the $\beta / \alpha$ ratio for each construct assayed. Amended with permission from American Society for Microbiology. Brown, R. C., S. Pattison, R. J. van Ree, E. Coghill, A. Perkins, S. M. Jane, and J. M. Cunningham. 2002. Distinct domains of erythroid Krüppel-like factor modulate chromatin remodeling and transactivation at the endogenous beta-globin gene promoter. Mol. Cell. Biol. 22:161-170/DOI:10.1128. 
an internal domain of EKLF is sufficient to activate $\beta$-globin gene expression to wildtype levels (Brown et al., 2002). These observations are contrasted with previous studies in which the first 104 amino acids of EKLF activated GAL4-dependent transcription (Chen \& Bieker, 1996). More importantly, our studies indicate EKLF contains separable chromatin remodeling and transactivation domains.

\section{PROJECT OUTLINE}

I joined Dr. Cunningham's laboratory with a strong interest in studying globin gene regulation to understand the molecular mechanism underlying one of the most common hematological disorders, $\beta$-thalassemia. Success in this endeavor would identify therapeutic targets that would reverse or delay the globin gene switch, thus ameliorating the $\beta$-thalassemic or sickle cell disease phenotype.

Based on the cellular studies by previous colleagues in the laboratory, I propose to examine the role of the newly defined chromatin remodeling domain of EKLF $(\triangle 221 \mathrm{EKLF})$ in a whole animal model that allows the analysis of modulation of gene expression and chromatin structure. My working hypothesis is that expression of the chromatin remodeling domain of EKLF, in the absence of the transactivation domain, is necessary and sufficient for altering the chromatin structure at the $\beta$-globin locus in vivo. A corollary hypothesis is that expression of the chromatin remodeling domain alone is not sufficient for activation of $\beta$-globin gene transcription in vivo. I plan to generate specific mutants of the EKLF gene at the endogenous locus in a murine knock-in model to address the following specific aims.

Specific aim 1: To characterize the cellular consequences on murine hematopoiesis of expression of the knock-in allele in vivo.

The goal of this specific aim is to provide an initial analysis of the cellular effects of $\triangle 221 \mathrm{EKLF}$ expression in a murine model. First, matings between $\triangle 221 \mathrm{EKLF}$ heterozygous male and female animals will be established to determine if $\triangle 221 \mathrm{EKLF}$ homozygous animals are viable. If no adult $\triangle 221 \mathrm{EKLF}$ homozygous animals are observed, I will dissect embryos at different time points during gestation to determine when and how $\triangle 221 \mathrm{EKLF}$ animals die. I hypothesize that animals expressing $\triangle 221$ EKLF die of a lethal anemia similar to the EKLF knock-out mice. Similarly, I predict that cells expressing $\triangle 221 E K L F$ fail to execute normal terminal differentiation. These studies should provide valuable information on the role of the chromatin remodeling domain of EKLF in transactivation of $\beta$-globin gene transcription in vivo. 
Specific aim 2: To evaluate the functional consequences of expression of the knock-in allele on EKLF-dependent erythroid gene transcription.

I propose to explore the effects of $\triangle 221 \mathrm{EKLF}$ expression on gene transcription in vivo. Utilizing real time RT-PCR, I will quantify the transcription of the $\beta$-globin gene and putative non- $\beta$-globin EKLF-target genes to determine whether any of these genes require the chromatin remodeling properties of EKLF alone for gene transcription. Based upon our laboratory's cellular studies, my overarching hypothesis is that expression of the chromatin remodeling domain in the absence of the transactivation domain is not sufficient to activate gene transcription. Together, these results will provide insights into the role of EKLF in coordinating gene transcription at the $\beta$-globin locus and other gene loci.

Specific aim 3: To explore the functional consequences of expression of the knock-in allele on chromatin structure at the $\beta$-globin locus.

The studies proposed in this aim will directly test my working hypothesis that expression of $\triangle 221 \mathrm{EKLF}$ is sufficient for altering chromatin structure at the $\beta$-globin locus. First, I will analyze the nucleosomal remodeling of the $\beta$-globin promoter as measured by DNase I hypersensitivity. Subsequently, I will investigate the histone acetylation and methylation patterns at the $\beta$-globin locus utilizing quantitative chromatin immunoprecipitation (ChIP). The proposed studies should corroborate the observations in our EKLF-dependent erythroblast model and provide insight into the role of chromatin remodeling in transactivation of $\beta$-globin gene transcription.

In this dissertation, I describe the generation of the knock-in alleles of EKLF and the consequences of expression of the $\triangle 221 \mathrm{EKLF}$ in erythroid and megakaryocytic differentiation. In Chapter 2, I will describe the construction of gene targeting vectors utilizing recombineering technology and the generation of $\triangle 221 \mathrm{EKLF}$ heterozygous animals. In Chapter 3, I will report on the molecular consequences of expression of $\triangle 221 \mathrm{EKLF}$ as it relates to chromatin remodeling and transcriptional activation of $\beta$ globin. In Chapter 4, I will report on the novel role for EKLF in megakaryocytic differentiation. 


\section{CHAPTER 2: GENERATION OF A KNOCK-IN ALLELE OF EKLF UTLIZING RECOMBINEERING TECHNOLOGY}

\section{INTRODUCTION}

Genetically engineered mouse models have proven to be useful tools for many applications in research, medicine, and biotechnology. The approaches to generating these different mouse models have traditionally been based on the over-expression or ablation of a gene using transgenic and knock-out strategies, respectively (Roebroek et al., 2002). More recently, by using a knock-in approach and placing the expression of an exogenous gene under the transcriptional control of cis-acting elements belonging to the endogenous gene, investigators are able to study the gene in a more subtle manner (Roebroek et al., 2002).

Methods used for genetic engineering have relied mostly on a conventional approach. Restriction enzymes and DNA ligases are used to clone an appropriate piece of DNA sequences into a targeting vector. The major limitation of this strategy is the difficulty and time it takes to generate this vector utilizing large fragments of DNA (Copeland et al., 2001; Liu et al., 2003). At the time that I initiated my dissertation studies, a new and highly efficient method for manipulating the mouse genome had been developed. Termed recombineering, this method relies on the lambda phage-based homologous recombination in Escherichia coli to construct the targeting vector (Yu et al., 2000; Liu et al., 2003). Using this technology, it is possible to introduce large doublestranded DNA (dsDNA) fragments into DNA cloned on plasmids, bacterial artificial chromosomes (BACs), or P1 artificial chromosomes (PACs) via homologous recombination without the need for restriction enzymes or DNA ligases (Copeland et al., 2001; Liu et al., 2003). Additional advantages of using this new technology are speed, efficiency, and reliability.

Originally, recombineering has been utilized in yeast due to its efficient DNA double-stranded-break-and-repair recombination pathway, allowing the creation of recombinant DNA molecules by homologous recombination (Baudin et al., 1993). These recombination pathways allow efficient recombination of transformed linear, doublestranded DNA (dsDNA) with homologous sites in the yeast genome. Moreover, proficient recombination occurs even with only short stretches of homologous sequence, thereby allowing recombinant DNA to be generated in vivo without the use of restriction enzymes and DNA ligases (Baudin et al., 1993). Unlike in yeast, dsDNA is unstable in $E$. coli due to the presence of RecBCD, an ATP-dependent exonuclease that degrades dsDNA. However, E. coli strains that lack RecBCD can be transformed by linear dsDNA (Baudin et al., 1993).

There are disadvantages to utilizing yeast and yeast artificial chromosomes (YACs) in recombineering. First, YACs are less stable in their yeast host, in which recombination is potent and always active. Thus, undesired deletions and gene rearrangements are a barrier to using this organism. In contrast, bacterial artificial 
chromosomes (BACs) are stable in E. coli (Shizuya et al., 1992; Copeland et al., 2001). Moreover, YAC DNA is more difficult to purify compared to BAC DNA (Copeland et al., 2001). A yeast cell may contain both wild-type and modified YACs (Peterson et al., 1997), whereas a bacterial cell typically contains a single BAC (Copeland et al., 2001). Finally, manipulating recombinant YACs that are generated in yeast can be laborious and usually requires the YACs to be transferred to $E$. coli for subsequent manipulation, whereas BAC modification occurs directly in E. coli (Copeland et al., 2001).

Recombineering in E. coli can be accomplished by making use of lambda phage's homologous recombination proteins, called Red, which allow linear dsDNA fragments to be inserted via homologous recombination into DNA cloned on plasmids (Yu et al., 2000; Cotta-de-Almeida et al., 2003; Zhang \& Huang, 2003). To generate a recombinogenic strain of bacteria, a defective lambda prophage which lacks lysis and replication functions but retains the Red proteins is inserted into the bacterial genome (Yu et al., 2000). The phage genes of interest, exo, bet, and gam, are transcribed from the $\lambda P \mathrm{~L}$ promoter. This promoter is repressed by the temperature-sensitive repressor $c 1857$ at $32^{\circ} \mathrm{C}$. In contrast, derepression, that is the repressor is inactive, occurs at $42^{\circ} \mathrm{C}(\mathrm{Yu}$ et al., 2000). At low temperatures (i.e., $32^{\circ} \mathrm{C}$ ) no recombination proteins are produced. However, following a temperature shift to $42^{\circ} \mathrm{C}$ for as little as $15 \mathrm{~min}$, these proteins are expressed at high levels. The 5'-3' exonuclease, exo, creates single-stranded overhangs on introduced linear DNA; bet protects these overhangs and assists in the subsequent recombination process. Degradation of linear DNA is protected by gam, which inhibits the E. coli RecBCD protein (Yu et al., 2000).

Following induction of the recombination genes, linear dsDNA such as PCR products and oligonucleotides with sufficient homology in the $5^{\prime}$ and $3^{\prime}$ ends to a target DNA molecule already present in the bacteria (plasmid, BAC, or the bacterial genome itself) can be introduced into heat-shocked and electrocompetent bacteria using electroporation. The introduced DNA is modified by exo and bet and undergoes homologous recombination with the target molecule. The method is so efficient that coelectroporation of a supercoiled plasmid and a linear piece of DNA into heat-shocked, electrocompetent bacteria will work as well (Yu et al., 2000; Copeland et al., 2001; Liu et al., 2003).

This chapter describes the generation of knock-in mutant alleles of EKLF using the recent recombineering technology that encompasses a phage-based E. coli homologous recombination system. Several groups have used this new form of genetic engineering to construct standard, conditional, and knock in gene targeting vectors to modify murine embryonic stem (ES) cells (Lui et al., 2003; Zhou et al., 2004). Utilizing this method, I have constructed three targeting vectors in which cDNA encoding three different truncation mutations of EKLF ( $\triangle 164 \mathrm{EKLF}, \triangle 221 \mathrm{EKLF}$, and $\triangle 253 \mathrm{EKLF}$ ) have been inserted into the endogenous murine EKLF locus. The construction of all three targeting vectors was completed without the constraints of restriction sites and took a shorter time than a similar strategy utilizing traditional subcloning methods.

Subsequently, the targeting vectors were used to target the endogenous murine EKLF locus in ES cells. The animals I have generated should prove to be useful tools for 
analyzing the distinct molecular functions of EKLF in vivo.

Based upon recommendations from my graduate committee, I have chosen to focus my dissertation studies on characterizing one strain of mice while the other two strains are studied by my colleagues in the laboratory. I have chosen to focus on the $\triangle 221 \mathrm{EKLF}$ strain for several reasons. First, previous studies by my laboratory colleagues have demonstrated that the $\triangle 221 \mathrm{EKLF}$ domain is sufficient to alter local chromatin structure at the endogenous $\beta$-globin promoter; however this domain alone is insufficient to transactivate $\beta$-globin gene transcription to wild-type levels in a cellular model. Thus, the strain of mice expressing $\triangle 221 \mathrm{EKLF}$ is the most ideal model to elucidate the role of EKLF in chromatin remodeling and gene activation in vivo.

\section{MATERIALS AND METHODS}

\section{BAC transfer into recombinogenic strains}

An EKLF BAC clone in DH10B was obtained from the BACPAC Resources Center at Children's Research Hospital Oakland Research Institute (CHORI). The EKLF BAC DNA (75kb) was purified using the Miniprep DNA kit (Qiagen) as previously described (Liu et al., 2003). Briefly, E. coli cells encoding BACs were grown overnight in LB broth $(5 \mathrm{~mL})$ with chloramphenicol. Cells were collected by centrifugation at maximum speed (12,000 x g or 13, $000 \mathrm{rpm})$ and resuspended in buffer P1 (250 $\mu \mathrm{L})$. Buffer P2 $(250 \mu \mathrm{L})$ and buffer P3 $(350 \mu \mathrm{L})$ were added to each tube, and the tubes were spun for $4 \mathrm{~min}$ at $12,000 \mathrm{x} \mathrm{g}$. The supernatant was transferred to a new $1.5 \mathrm{~mL}$ tube and cleared by centrifugation for another $4 \mathrm{~min}$. Isopropanol $(750 \mu \mathrm{L})$ was added to the mixture, and DNA was precipitated at room temperature for $10 \mathrm{~min}$. The DNA was collected by spinning the tube for $10 \mathrm{~min}$ at the maximal speed, washed once with $70 \%$ ethanol $(1.0 \mathrm{~mL})$, air dried, and resuspended in TE $(50 \mu \mathrm{L})$. The purified EKLF BAC DNA (100-200 ng) was electroporated into the recombinogenic E. coli strain EL350 (a kind gift from Dr. Neal Copeland) using a BIO-RAD electroporator at $1.75 \mathrm{kV}, 25 \mu \mathrm{F}$ with the pulse controller set to $200 \Omega$ and time constant between 4.3-4.7. Transformed colonies were recovered on LB agar with $40 \mu \mathrm{g} / \mathrm{mL}$ chloramphenicol. The EKLF BAC DNA prepared from the original DH10B and transformed EL350 bacteria was digested with BamHI, EcoRI, and HindIII and separated on agarose gels to confirm that no DNA rearrangements had occurred during the BAC transfer.

\section{Plasmids}

The EKLF BAC retrieval plasmid was generated by ligating PCR product $\mathrm{AB}$ (left arm, EcoRI/BamHI), PCR product XY (right arm, BamHI/XbaI), and MC1-TK/polIIDT (VP101, EcoRI/XbaI) using T4 DNA ligase (Promega). The mini-targeting plasmid was generated in two-steps. First, PCR product EF (BglII/NotI) was ligated with a floxed $\mathrm{Neo}$-containing vector, a kind gift from Dr. Neal Copeland (PL452, BamHI/NotI). Then 
PCR product CD (SalI/HindIII) and HA- $\triangle 221 \mathrm{EKLF}$ cDNA-containing fragment (pspHAEKLF-C3, HindIII/EcoRI) were ligated with the vector containing the Neo cassette and PCR product EF generated in the first step. The ligation product was transformed into chemically competent DH5 $\alpha$ cells (Invitrogen) and plated on selective media containing both kanamycin $(50 \mu \mathrm{g} / \mathrm{mL})$ and carbenicillin $(100 \mu \mathrm{g} / \mathrm{mL})$. Only bacterial cells propagating the subcloned vector with the Amp and Neo resistance genes should grow under these conditions.

PCR products were amplified using ROCHE Expand High-Fidelity PCR System using $50 \mathrm{ng}$ of BAC DNA following the manufacturer's recommendations. PCR was performed using an MJ Research PCR machine with the following settings: $94^{\circ} \mathrm{C}$ for 2 min, then 10 cycles of $94^{\circ} \mathrm{C}$ for $15 \mathrm{sec}, 55^{\circ} \mathrm{C}$ for $30 \mathrm{sec}$, and $72^{\circ} \mathrm{C}$ for $45 \mathrm{sec}$. This was followed by 15 cycles of $94^{\circ} \mathrm{C}$ for $15 \mathrm{sec}, 55^{\circ} \mathrm{C}$ for $30 \mathrm{sec}, 72^{\circ} \mathrm{C}$ for $45 \mathrm{sec}$, with an additional $5 \mathrm{sec}$ extension time each cycle. To check the PCR reaction, $5 \mu \mathrm{L}$ of the $50 \mu \mathrm{L}$ PCR reaction mixture was loaded onto an agarose gel. The remaining $45 \mu \mathrm{L}$ of PCR product was purified using the QIAGEN PCR Purification Kit.

\section{Recombineering}

Recombineering was performed as previously described (Liu et al., 2003). To retrieve the gap-repaired plasmid, EL350 cells containing the EKLF BAC was grown at $32^{\circ} \mathrm{C}$ to an $\mathrm{OD}_{600}=0.5$ in LB broth with chloramphenicol. The cells were transferred to and shaken in a $42^{\circ} \mathrm{C}$ water bath for $15 \mathrm{~min}$ to induce expression of the $\lambda$ recombination proteins, and quickly chilled in ice water for $10 \mathrm{~min}$. Electrocompetent cells were prepared by washing the cells three times with ice cold water. Finally, the cell pellet was resuspended in ice cold water $(50 \mu \mathrm{L})$ and electroporated with the BamHI-linearized retrieval vector (1-2 $\mu \mathrm{L})$. After electroporation, $1 \mathrm{~mL}$ of $\mathrm{LB}$ medium was added to the cuvette, and the culture was incubated at $32^{\circ} \mathrm{C}$ for $1 \mathrm{~h}$ with shaking. The cells were then plated on agar plates with the appropriate antibiotic.

For targeting, frozen EL350 electrocompetent cells previously prepared in the laboratory were used. The frozen cells were thawed at room temperature and quickly put on ice. These cells were co-electroporated with the targeting cassette (100 ng) and the gap-repaired plasmid (10 ng) DNA as previously described (Liu et al., 2001). The targeting cassette was excised from the mini-targeting vector with NotI and SalI digest and purified by the QIAGEN Gel Purification System.

\section{Excision of the Neo cassette in bacteria}

Frozen EL350 cells previously prepared in the laboratory and induced for $\mathrm{Cre}$ expression by prior growth in arabinose-containing medium were used to test the excision of the floxed Neo cassette. The EL350 strain of E. coli was previously engineered to harbor an arabinose-inducible Cre gene ( $\left.P_{\mathrm{BAD}}-c r e\right)$ (Lee et al., 2001; Liu et al., 2003). Plasmid DNA (10-50 ng) was electroporated into frozen electrocompetent cells $(50 \mu \mathrm{L})$. 
LB medium $(1 \mathrm{~mL})$ was added to the cuvette, and the culture was shaken at $32^{\circ} \mathrm{C}$ for $1 \mathrm{~h}$. The cells were plated on ampicillin-containing media. DNA was extracted from selected ampicillin-resistant colonies and digested with restriction enzymes.

\section{Gene targeting in ES cells and generation of $\triangle 221 \mathrm{EKLF}$ mice}

ES cells were obtained from Specialty Media and maintained following the manufacturer's recommendations. For gene targeting, the NotI linearized HA$\Delta 221 \mathrm{EKLF}-\mathrm{KI}$ construct was electroporated into 129Sv ES cells (Specialty Media) and recombinants were selected in medium supplemented with G418 (Gibco) and ganciclovir (Syntex). Selection was continued for eight days and the surviving ES clones were picked and expanded for an additional four days. Genomic DNA was extracted from each clone and analyzed by Southern blotting to identify properly targeted ES clones. Sequences for 5' and 3' probes used in Southern blot analysis are available in Appendix Figure A.2.

Properly targeted ES clones with a normal karyotype were injected into C57BL/6 blastocysts and transferred into pseudopregnant females to generate chimeras (Transgenic Core Facility at St. Jude Children's Research Hospital). Male chimeras were mated with C57BL/6 wild-type females to generate F1 offsprings. To remove the floxed Neo cassette, $\triangle 221 \mathrm{EKLF}$ heterozygous mice were mated with mice harboring the cre transgene diallelically expressed under the control of the adenovirus EIIa promoter that targets expression of Cre recombinase to the early mouse embryo (Jackson Labs).

\section{Mouse genotyping}

For Southern blotting, mouse tail DNA was digested with NheI (New England Biolabs), separated on $0.8 \%$ agarose gels, and transferred to GeneScreen Plus hybridization transfer membrane (Perkin Elmer). A 543 bp 3' external probe was used for hybridization. Genotyping by PCR was performed using a common WT and KI forward primer, (5'-ATGAGGCAGAAGAGAGAGAGGAG-3'), a WT reverse primer (96 bp; 5'TGAGTGTACTGATGGAGGGTAAGA-3'), and a KI reverse primer (500 bp; 5'CCGTGTGTTTCCGGTAGTG-3'). PCR primers for genotyping were designed using Primer Design in Clone Manager. PCR was performed on a BIORAD PCR machine with the following settings: $94^{\circ} \mathrm{C}$ for 3 min followed by 32 cycles of $94^{\circ} \mathrm{C}$ for $30 \mathrm{sec}, 60^{\circ} \mathrm{C}$ for $30 \mathrm{sec}$, and $72^{\circ} \mathrm{C}$ for $1 \mathrm{~min}$ with a final extension at $72^{\circ} \mathrm{C}$ for $10 \mathrm{~min}$. Products were visualized on agarose gel with ethidium bromide staining.

\section{Husbandry of mice}

The animals used in the studies were housed in the Animal Resources Center of St Jude Children's Research Hospital, Memphis, Tennessee, and the University of Chicago, Chicago, Illinois, according to approved Animal Care Use Protocols (ACUP). 


\section{Analysis of peripheral blood counts}

Blood was harvested from the retro-orbital sinus into EDTA-coated microcapillary tubes and submitted to the St Jude Children's Research Hospital Animal Diagnostic Laboratory for complete blood count (CBC) analysis including a peripheral blood smear.

\section{Primers}

PCR primers for recombineering were designed using Primer Design in Clone Manager or Primer 3. The primer sequences used for generating the HA- $\Delta 221 \mathrm{EKLF}-\mathrm{KI}$ targeting vector are:

AB-fragment (540 bp): Primer A: 5'-CGGAATTCGGTGCTGGTGGTTGTCTAGG-3' Primer B: 5'-CGCGGATCCTGTGGTTGCTTGTAATTGA-3'; XY-fragment (640 bp): Primer X: 5'-CGCGGATCCTACATAGCCTCTGTGCATTC-3'; Primer Y: 5'-GCTCTAGACACCGTCTCTAGGTCAGGTA-3'; CD-fragment (596 bp): Primer C: 5'-ACGCGTCGACCAAAGGGTCCCAAAGACCTTTC-3'; Primer D: 5'GGGTGGTGAGTGTACTGATG-3'; EF-fragment (541 bp): Primer E: 5' GGAAGATCTGGCAGGTGGTCTTGCATAGG-3'; Primer F: 5'ATAAGCGGCCGCAGTATTCAGGATGGGAGAAG-3'; Primers used to generate the fragment containing the HA- $\triangle 221 \mathrm{EKLF}$ sequence were Forward: 5'CATGCCATGGCCTATGACGTCCCAGATTACG-3'; Reverse: 5'AGTCAGGATATCCTACTTAGTCAAAGGTGGCGCTTCATGTGC-3’.

\section{RESULTS}

\section{Generation and characterization of targeting vectors}

To elucidate the role of the functional domains of EKLF in primary mouse erythroblasts, I proposed to generate animals expressing distinct domains of EKLF identified in previous cellular assays (Brown et al., 2002). Targeting vectors, in which cDNA encoding HA-tagged truncation mutant of EKLF linked in cis to a Neo selection cassette (HA- $\triangle 221 \mathrm{EKLF}-\mathrm{Neo}$ ) were generated, utilizing recombineering methods. The overall strategy is illustrated in Figure 2.1.

First, to facilitate the manipulation of the EKLF genomic locus by recombineering, it was necessary to retrieve a region of the mouse genome containing the EKLF gene and its $c i s$-regulatory sequences. To accomplish this, a vector capable of retrieving an $11 \mathrm{~kb}$ fragment spanning the EKLF locus was constructed by subcloning PCR amplified fragments $\mathrm{AB}$ and $\mathrm{XY}$ into an expression vector. These regions, $\mathrm{AB}$ and $\mathrm{XY}$, mark the 5' and 3' ends of the fragment to be subcloned by gap repair, respectively. Next, to obtain the gap-repaired plasmid containing our genomic region of interest (Figure 2.2A), the BamHI-linearized retrieval vector was electroporated into the recombinogenic bacterial 
Figure 2.1. Overview of the recombineering method used to generate vectors for targeting in ES cell. A BAC clone containing the EKLF genomic locus was transferred from the E. coli strain DH10B to the recombinogenic strain EL350. These cells were electroporated with a BamHI linearized retrieval plasmid containing PCR products, AB and XY, of homology to the EKLF BAC. By gap-repair, this plasmid rescued an $11 \mathrm{~kb}$ fragment containing the EKLF gene plus $3 \mathrm{~kb}$ of upstream sequences and $4.5 \mathrm{~kb}$ of downstream sequences. Cells containing the correct gap-repaired plasmid were electroporated with a fragment containing HA- $\Delta 221 \mathrm{EKLF}-$ floxed Neo flanked by homology sequence from the EKLF genomic locus. This fragment was excised from the mini-targeting vector in which PCR products CD, EF, and HA- $\triangle 221 \mathrm{EKLF}$ were subcloned into the PL452 plasmid containing a floxed Neo cassette. The resulting plasmid containing the modified EKLF locus with insertion of HA- $\triangle 221 \mathrm{EKLF}$-floxed NEO cassette into the ATG at amino acid 19 was linearized with NotI and electroporated into ES cells. EKLF contains three exons (E1-E3) as denoted by black boxes. AB (green) and XY (blue) are homologous to a region upstream and downstream of the EKLF locus, respectively. CD (red) and EF (orange) flank the fragment to be recombineered into the EKLF locus and are homologous to sequences in exon 1 of the EKLF gene. The CD region, containing the ATG start site, is placed in frame with HA$\Delta 221 \mathrm{EKLF}$ encoding sequences. Lox $\mathrm{P}$ sites are represented by black arrow heads. 

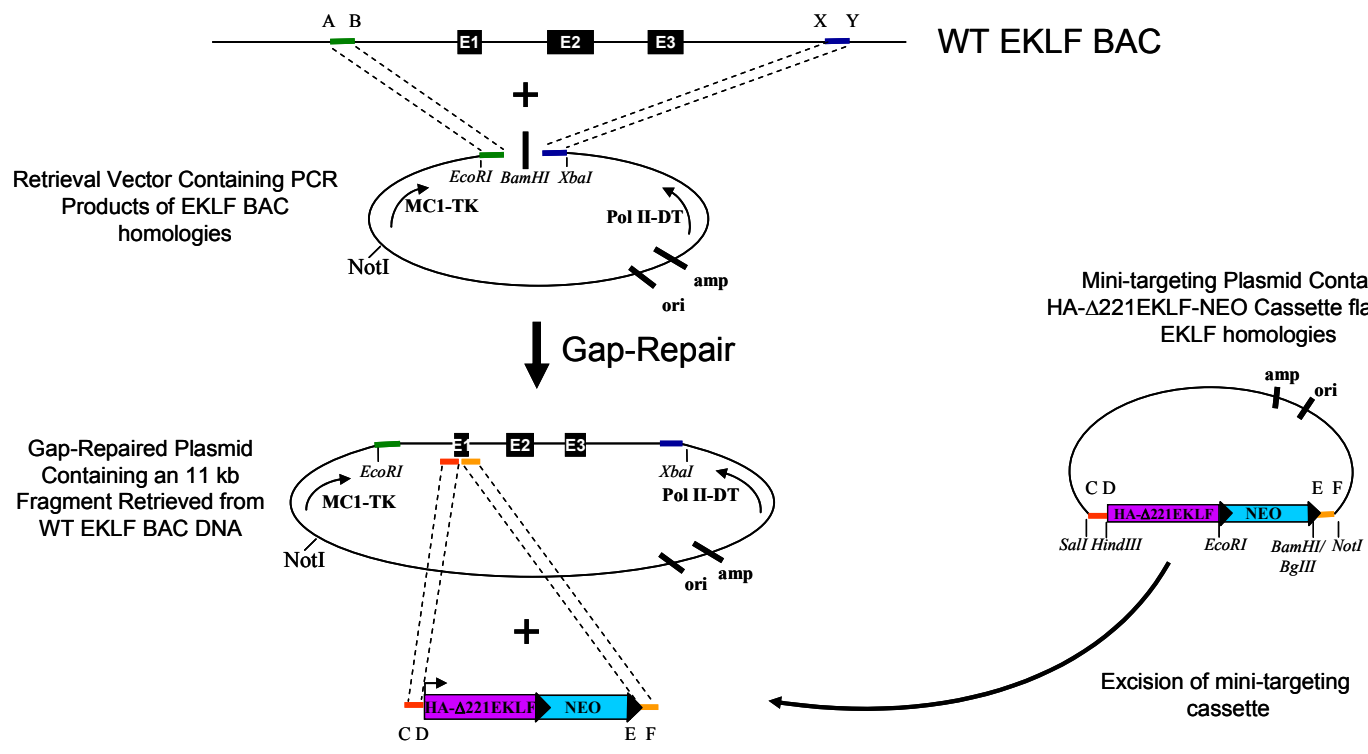

Mini-targeting Plasmid Containing HA- $\triangle$ 221EKLF-NEO Cassette flanked by EKLF homologies WT EKLF BAC DNA

Recombineering

$$
\text { cassette }
$$

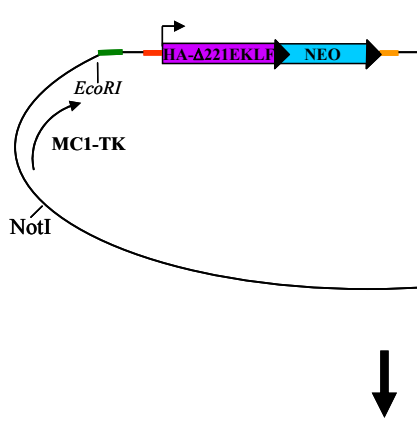

\section{Linearization of Recombineered Plasmid and Electroporation into ES cells}


A.

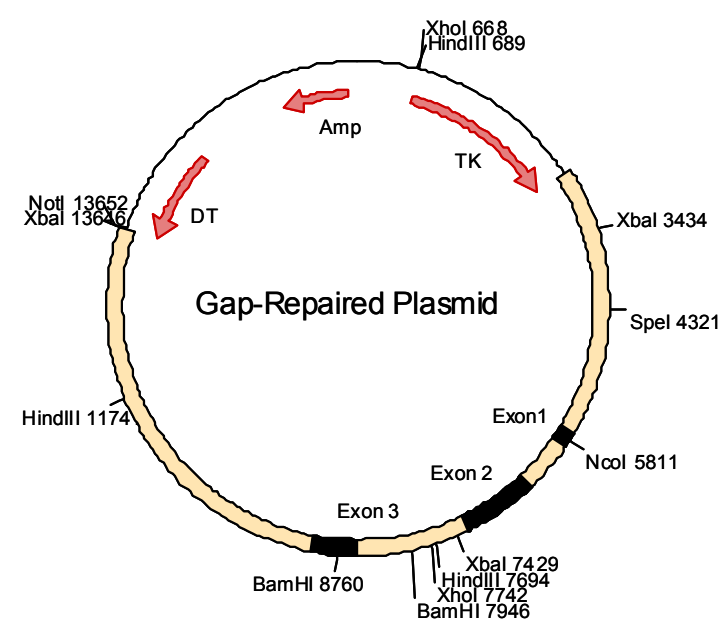

B.

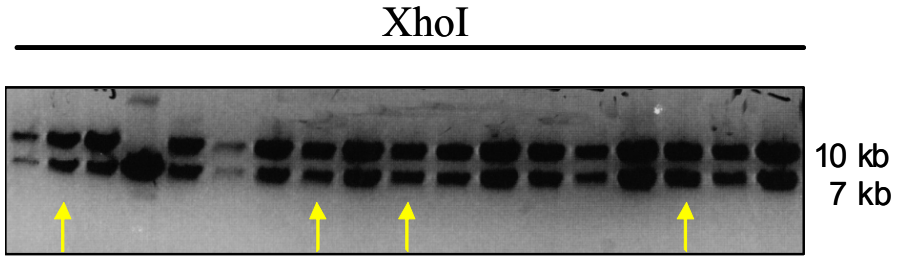

C.

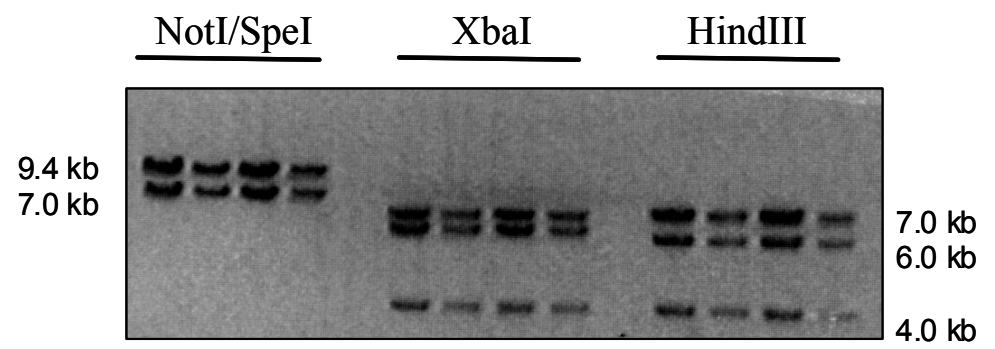

Figure 2.2. Mapping of the gap-repaired plasmid. (A) Diagram of the resultant gaprepaired plasmid obtained by electroporation of EL350 cells harboring EKLF BAC with a BamHI-linearized retrieval vector. DT $=$ diphtheria toxin; $\mathrm{TK}=$ tymidine kinase; $\mathrm{Amp}=$ Ampicillin (B) To confirm the capture of the $11 \mathrm{~kb}$ EKLF BAC with appropriate flanking sequences, DNA was isolated from 18 colonies and digested with XhoI (10 kb and $7 \mathrm{~kb})$. (C) Subsequently, DNA from 4 colonies (indicated by arrows from above) was digested with NotI/SpeI (9.4 kb, $7.7 \mathrm{~kb})$, XbaI (6.8kb, $6.3 \mathrm{~kb}, 4.0 \mathrm{~kb})$ and HindII $(7.0 \mathrm{~kb}, 6.0 \mathrm{~kb}$, $4.0 \mathrm{~kb})$, respectively. The expected bands are in parentheses. 
strain harboring the EKLF BAC. Since these bacterial cells had been engineered to express the $\lambda$ recombination system with induction at $42^{\circ} \mathrm{C}$, homologous recombination would occur resulting in the retrieval of the mouse genomic region flanked by the homology arms AB and XY. DNA from 18 ampicillin-resistant colonies were purified by the miniprep method and digested with XhoI to identify homologous recombinants. As shown in Figure 2.2B, DNA from 17 out of 18 colonies digested with XhoI showed the predicted $7 \mathrm{~kb}$ and $10 \mathrm{~kb}$ bands. The DNA in lane 4 appears to be undigested plasmid containing at least two topologically different forms of DNA, corresponding to supercoiled forms (bottom band) and nicked circles (upper band). DNA from four colonies (indicated by arrows) was selected for further restriction enzyme analysis. Digest with NotI/SpeI (9.4 kb / 7.7 kb), Xbal(7.0 kb / $6.0 \mathrm{~kb} / 4.0 \mathrm{~kb})$, and HindIII (7.0 $\mathrm{kb} / 6.0 \mathrm{~kb} / 4.0 \mathrm{~kb}$ ) produced fragments of the expected sizes (Figure 2.2C). Four DNA samples confirmed by restriction mapping were sent for sequencing at the Hartwell Center of St Jude Children's Research Hospital. One construct had numerous mutations and deletions near the junctions where homologous recombination took place while another construct had base pair substitutions within the EKLF gene. The gap-repaired plasmid with the correct sequences was selected for the next step in recombineering.

Next, a mini-targeting vector containing the knock-in fragment that will be used to target the gap-repaired plasmid was constructed by subcloning. Fragment EF which is homologous to the targeting site was generated by PCR, digested with BglII/NotI, and ligated with PL452 DNA digested with NotI/BamHI. The restriction enzymes BglII and BamHI have compatible ends (New England Biolabs). The floxed Neo gene in PL452 is expressed from a hybrid PGK-EM7 promoter (Liu et al., 2003). Expression of Neo from the PGK promoter permits selection of Neo resistant mammalian ES clones. On the other hand, EM7 drives Neo expression in bacterial cells facilitating selection of Neo resistant colonies. PCR was used to generate the CD fragment with SalI and HindIII sites and a fragment containing HA- $\Delta 221$ EKLF sequences with stop codons in all three reading frames and addition of HindIII and EcoRI restriction sites. Homology arm CD was designed carefully so that the HA- $\triangle 221$ EKLF cDNA would be inserted into the endogenous ATG start site, placing the mutant gene under the cis-regulatory control of the endogenous locus.

To complete subcloning the mini-targeting vector (Figure 2.3A), SalI/HindIIIdigested CD fragment and HindIII/EcoRI-digested HA- $\triangle 221 \mathrm{EKLF}$ fragment were ligated with SalI/EcoRI-digested PL452-EF plasmid DNA. The ligation product was transformed into DH5 $\alpha$ competent cells and plated on kanamycin/ampicillin selective media. Colonies propagating the subcloned plasmid with the Amp and Neo resistant genes should only grow under these conditions. Restriction analysis with EcoRI digest identified six out of eighteen colonies that were homologous recombinants (Figure 2.3B, as indicated by arrows). DNA from these six colonies was subjected to further restriction digestion analysis with NotI/SalI (3.5 kb, $2.9 \mathrm{~kb})$, NheI (5.8 kb, $0.6 \mathrm{~kb})$, XhoI (3.4 kb, 3.0 $\mathrm{kb}$ ), and HindIII/EcoRI (3.7 kb, $2.2 \mathrm{~kb}, 0.6 \mathrm{~kb}$ ) (Figure 2.3C). Three of the six colonies showed the correct restriction patterns. Before proceeding with the final step, the minitargeting vector was extensively analyzed by restriction enzyme digestion (Appendix Figure A.1; Table A.2). Furthermore, sequence analysis of the junctions between CD- 
A.

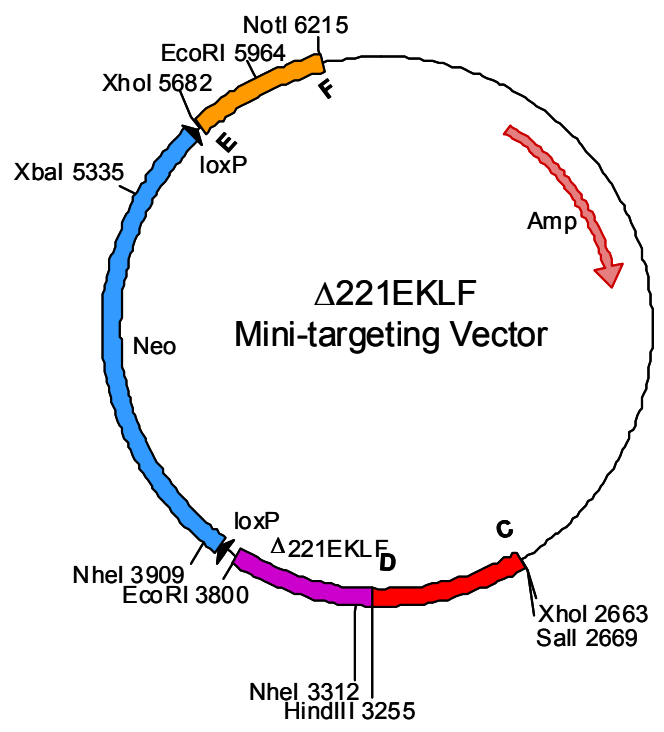

B.

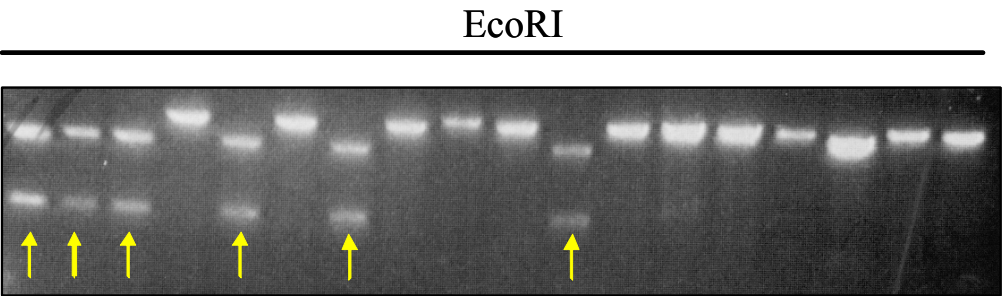

$4.3 \mathrm{~kb}$

$2.2 \mathrm{~kb}$

C.

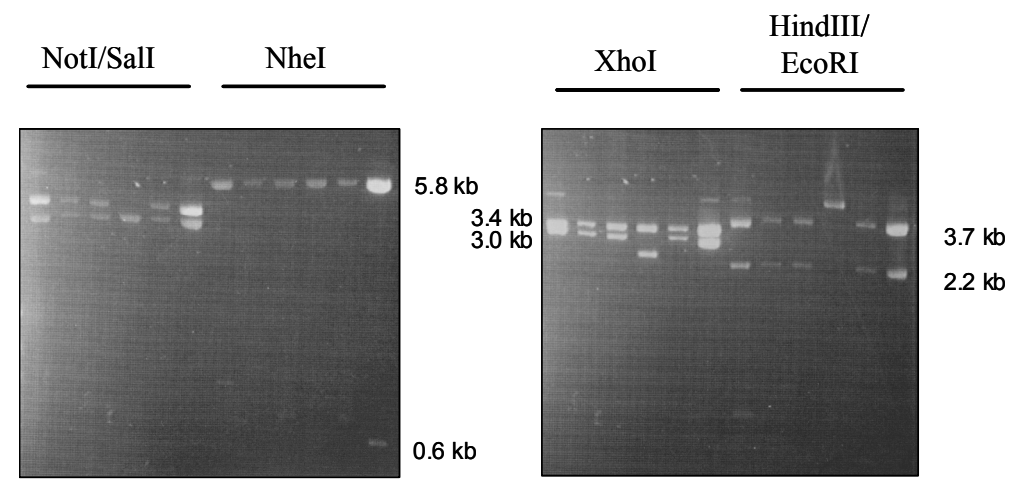

Figure 2.3. Analysis of $\Delta \mathbf{2 2 1 E K L F}$ mini-targeting vector. (A) Plasmid map of HA$\triangle 221 \mathrm{EKLF}$ mini targeting vector with restriction enzyme sites. $\mathrm{CD}, \mathrm{EF}=$ homologies to EKLF gene; Amp = Ampicillin; Neo = Neomycin (B) DNA from 18 kanamycin resistant colonies were prepared and digested with EcoRI (4.3 kb, $2.2 \mathrm{~kb})$. (C) DNA from the six correctly identified colonies (in lanes $1,2,3,5,7$, and 11 panel B) was further digested with NotI/SalI (3.5 kb, $2.9 \mathrm{~kb})$, NheI (5.8 kb, $0.6 \mathrm{~kb})$, XhoI (3.4 kb, $3.0 \mathrm{~kb})$, and HindIII/EcoRI (3.7 kb, $2.2 \mathrm{~kb}, 0.6 \mathrm{~kb})$, respectively. The predicted fragments for the correctly targeted vector are in parentheses 
HA- $\Delta 221 \mathrm{EKLF}-N e o-\mathrm{EF}$ confirmed that the predicted sequence was present.

Finally, to obtain the final targeting vector (Figure 2.4A) that will be used for targeting in ES cells, the complete mini-targeting cassette was introduced into the gaprepaired plasmid by recombineering. The mini-targeting cassette containing CD-HA$\Delta 221 \mathrm{EKLF}-\mathrm{Neo}$-EF was first excised from PL452 by NotI/SalI digest and gel-purified. Then, the purified cassette (300 ng) was co-electroporated along with the gap-repaired plasmid DNA (50 ng) into EL350 cells, which had been induced for Red recombination at $42^{\circ} \mathrm{C}$. Subsequently, the bacterial cells were plated on ampicillin/kanamycin selective media. No growth was visible after $24-48 \mathrm{hr}$ incubation at $32^{\circ} \mathrm{C}$. Co-electroporation was then attempted with varying amounts of targeting cassette (100-500 ng) and gap-repaired DNA (10-100 ng). Several ampicillin/kanamycin-resistant colonies were analyzed, however, there were no correct recombinants. I then decided to perform sequential electroporation. First, the gap-repaired DNA was electroporated into EL350 cells. The colonies were selected and the DNA digested with restriction enzymes to identify the bacterial colony containing the gap-repaired plasmid. Next, the mini-targeting cassette was electroporated into EL350 cells containing the gap-repaired plasmid. These cells had been induced for the Red recombination genes by a short incubation at $42^{\circ} \mathrm{C}$ to allow homologous recombination to occur. This resulted in modification of the EKLF genomic locus contained in the gap-repaired plasmid by targeting the mini-cassette to the start site of EKLF.

Prior to gene targeting in ES cells, the final targeting vector was characterized structurally and functionally. First, to screen for aberrant gene rearrangements, DNA from the gap-repaired plasmid (denoted 1), $\triangle 164 \mathrm{EKLF}$ targeting vector (denoted 2), $\triangle 221 \mathrm{EKLF}$ targeting vector (denoted 3), and $\triangle 253 \mathrm{EKLF}$ targeting vector (denoted 4) were digested with numerous restriction enzymes. Shown in Figure 2.4A are the restriction enzyme patterns after digestion with EcoRI, HindIII, XhoI, XbaI, AccIII, KpnI, SpeI, and PvuI, respectively. All samples displayed the expected digestion pattern by agarose gel electrophoresis. The predicted fragments are listed in Table 2.1. Next, to evaluate for mutations that may have occurred during recombineering, DNA from each targeting vector was submitted to the Hartwell Center at St Jude Children's Research Hospital for sequence analysis and confirmed that the targeting vectors had the correct sequences.

Finally, to ensure that the loxP sites in the targeting vectors would properly recombine in the presence of cre recombinase thereby removing the Neo cassette, the targeting vectors were electroporated into EL350 cells, which had been induced for cre expression by prior growth in arabinose-containing medium. Ampillicin-resistant colonies were picked and the DNA from these colonies was digested with SpeI, HindIII, BamHI, and NheI, respectively (Figure 2.4B, data shown for $\triangle 221 \mathrm{EKLF}$ only). After cre induction, there is a loss of SpeI and NheI sites resulting in only one band. Digestion with HindIII and BamHI leads to a smaller product due to excision of the Neo cassette as demonstrated by one of the bands being shifted lower (i.e., lower molecular weight). 
A.
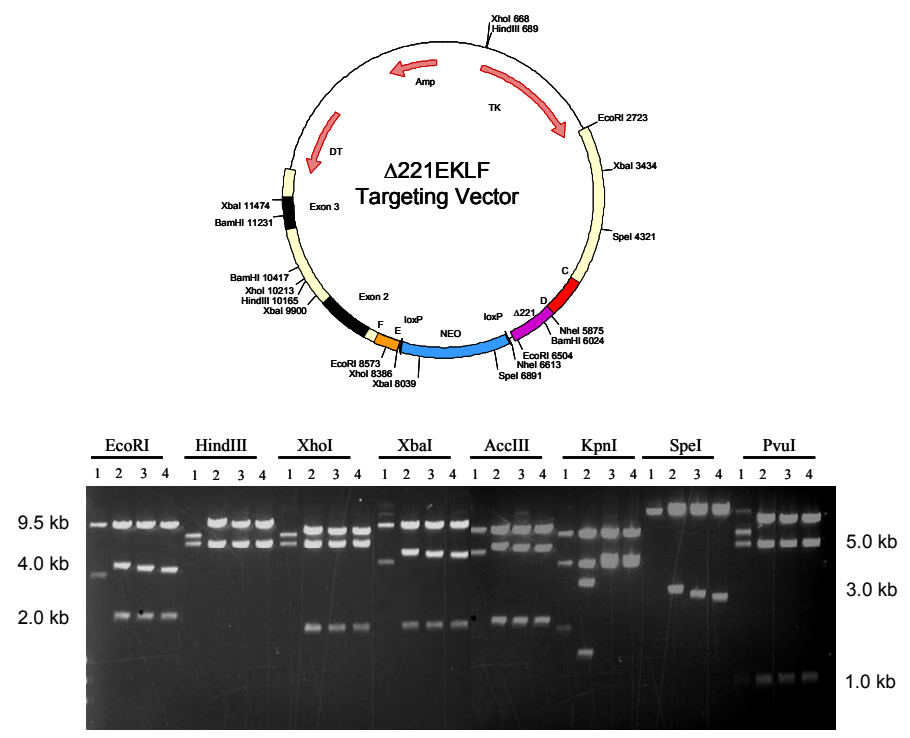

B.
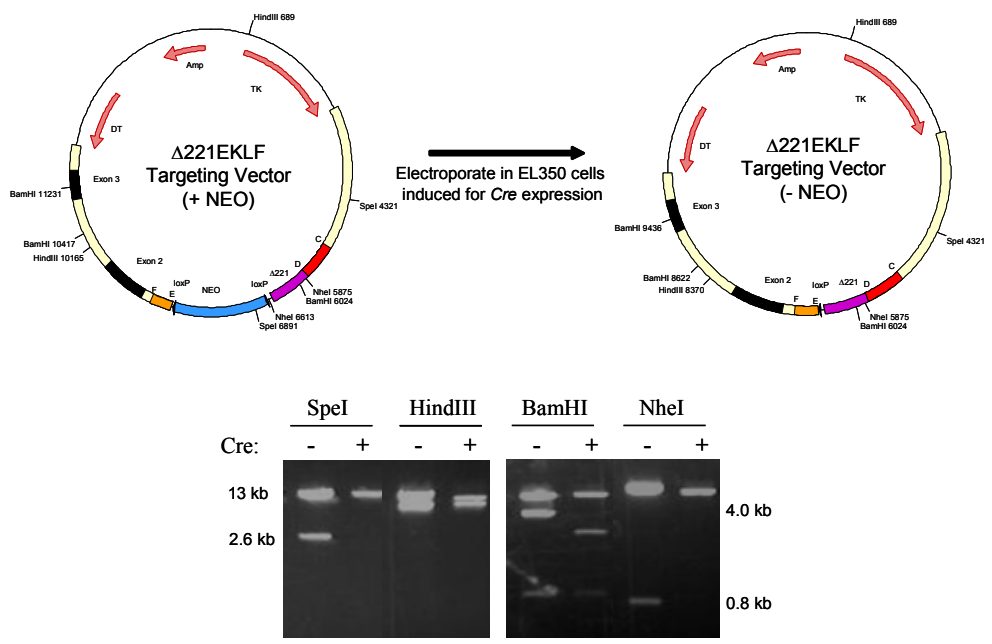

Figure 2.4. Verification of the final targeting vector using restriction enzyme analysis. (A) Plasmid map of the HA- 2221 EKLF final targeting vector. DNA was prepared from ampicillin/kanamycin-resistant colonies and subjected to digestion with various enzymes. Lane 1 = retrieval vector containing $11 \mathrm{~kb}$ EKLF BAC; lane $2=\mathrm{HA}-$ $\Delta$ 164EKLF; lane $3=\mathrm{HA}-\Delta 221 \mathrm{EKLF}$; lane $4=\mathrm{HA}-\Delta 153 \mathrm{EKLF}$. (B) Plasmid maps of the HA- $\triangle 221 E K L F$ final targeting vector with and without Neo. The final targeting vector was electroporated in EL350 cells induced for cre expression and ampicillin-resistant colonies were picked. DNA from the colonies was digested with several restriction enzymes. $\mathrm{CD}, \mathrm{EF}=\mathrm{EKLF}$ homologies; $\mathrm{DT}=$ diphtheria toxin; $\mathrm{TK}=$ thymidine kinase; Amp $=$ Ampicillin; $\mathrm{Neo}=$ Neomycin . 
Table 2.1. Predicted fragment size with restriction enzyme digestion.

\begin{tabular}{|c|c|c|c|c|}
\hline Enzyme & $\begin{array}{c}\text { Retrieval (1) } \\
\text { plasmid }\end{array}$ & $\Delta 164$ & $\Delta 221$ & $\Delta 253$ \\
\hline EcoRI & $\begin{array}{l}9.5 \\
3.4\end{array}$ & $\begin{array}{l}9.6 \\
4.0 \\
2.1\end{array}$ & $\begin{array}{l}9.5 \\
3.8 \\
2.1\end{array}$ & $\begin{array}{l}9.4 \\
3.7 \\
2.1\end{array}$ \\
\hline HindIII & $\begin{array}{l}7.0 \\
5.9\end{array}$ & $\begin{array}{l}9.6 \\
5.9\end{array}$ & $\begin{array}{l}9.5 \\
5.9\end{array}$ & $\begin{array}{l}9.4 \\
5.9\end{array}$ \\
\hline XhoI & $\begin{array}{l}7.3 \\
5.8\end{array}$ & $\begin{array}{l}7.9 \\
5.8 \\
1.8\end{array}$ & $\begin{array}{l}7.7 \\
5.8 \\
1.8\end{array}$ & $\begin{array}{l}7.6 \\
5.8 \\
1.8\end{array}$ \\
\hline $\mathrm{XbaI}$ & $\begin{array}{l}7.3 \\
5.6\end{array}$ & $\begin{array}{l}8.9 \\
4.8 \\
1.9\end{array}$ & $\begin{array}{l}8.9 \\
4.6 \\
1.9\end{array}$ & $\begin{array}{l}8.9 \\
4.5 \\
1.9\end{array}$ \\
\hline AccIII & $\begin{array}{l}8.0 \\
4.9\end{array}$ & $\begin{array}{l}8.0 \\
5.5 \\
2.0\end{array}$ & $\begin{array}{l}8.0 \\
5.4 \\
2.0\end{array}$ & $\begin{array}{l}8.0 \\
5.3 \\
2.0\end{array}$ \\
\hline KpnI & $\begin{array}{l}7.1 \\
4.0 \\
1.8\end{array}$ & $\begin{array}{l}7.1 \\
4.0 \\
3.0 \\
1.4\end{array}$ & $\begin{array}{l}7.1 \\
4.3 \\
4.0\end{array}$ & $\begin{array}{l}7.1 \\
4.2 \\
4.0\end{array}$ \\
\hline SpeI & 12.9 & $\begin{array}{r}12.8 \\
2.7\end{array}$ & $\begin{array}{r}12.8 \\
2.6\end{array}$ & $\begin{array}{r}12.8 \\
2.5\end{array}$ \\
\hline PvuI & $\begin{array}{l}6.6 \\
5.2 \\
1.0\end{array}$ & $\begin{array}{l}9.3 \\
5.2 \\
1.0\end{array}$ & $\begin{array}{l}9.1 \\
5.2 \\
1.0\end{array}$ & $\begin{array}{l}9.0 \\
5.2 \\
1.0\end{array}$ \\
\hline
\end{tabular}




\section{Targeting of the murine EKLF genomic locus}

A targeting vector was generated to introduce the $\triangle 221 \mathrm{EKLF}$ encoding cDNA at the start ATG of the endogenous EKLF gene (Figure 2.5A). EKLF has only 3 exons (Miller \& Bieker, 1993). In my targeting strategy, exons 2 and 3 of the endogenous gene were left intact. Stop codons were placed in all three reading frames to ensure proper translation of the knock-in allele. The 5' and 3' homology regions were $3 \mathrm{~kb}$ and $4 \mathrm{~kb}$, respectively. MC1-thymidine kinase and PolII-diptheria toxin cassettes were included for double negative selection in the ES cells. This selection strategy has been successfully utilized in our laboratory to enrich for ES clones that have undergone homologous recombination, as opposed to random integration (V.P. personal communication). The data shown here are specific to HA- $\triangle 221 \mathrm{EKLF}$, however, targeting for HA- $\Delta 164 \mathrm{EKLF}$ and HA- $\triangle 253 \mathrm{EKLF}$ were completed in a similar fashion.

To replace the wild-type EKLF gene with HA- $\Delta 221 \mathrm{EKLF}$ in ES cells, the targeting vector was linearized by NotI digestion and electroporated into $129 / \mathrm{SvEv}$ ES cells. After eight days of selection in G418 and ganciclovir, ES cell clones were picked and expanded. Genomic DNA from these clones was digested with EcoRV or NheI and analyzed by Southern blot analysis with 5' and 3' probes, respectively. The wild-type EKLF allele is detected as an $8.7 \mathrm{~kb}$ fragment with the 5' probe and as an $11.1 \mathrm{~kb}$ fragment with the 3' probe. The recombinant allele is detected as a $9.1 \mathrm{~kb}$ fragment with the 5' probe and as a $6.8 \mathrm{~kb}$ fragment with the 3 ' probe because of the introduction of novel EcoRV and NheI sites into the 5' and 3' regions of the genes, respectively, in the targeting vector. As shown in Figure 2.5B, ES DNA in lanes 3-7 of the top panel contain the $8.7 \mathrm{~kb}$ wild-type allele and the $6.8 \mathrm{~kb}$ knock-in allele with the 5' probe, whereas lanes 1-2 are wild-type ES cell DNA containing only an $8.7 \mathrm{~kb}$ fragment. Similarly, ES cell DNA in lanes 3-6 of the bottom panel contain the $11.1 \mathrm{~kb}$ wild-type allele and the $9.1 \mathrm{~kb}$ knock-in allele with the 3' probe, and lanes 1-2 are wild-type ES cell DNA containing only an $11.1 \mathrm{~kb}$ fragment. A total of $223 \mathrm{ES}$ clones were screened, and 17 positive clones were identified ( $\sim 8 \%$ recombinants).

\section{$\triangle 221 E K L F$ heterozygous mice are phenotypically normal}

ES cells properly targeted with $\Delta 221 \mathrm{EKLF}$ were injected into C57Bl/6 wild-type blastocysts to generate $\Delta 221 \mathrm{EKLF}$ chimeras. Male chimeras whose genetic composition is derived from the ES cells and donor blastocysts were mated with female C57B1/6 mice to transmit the knock-in allele. To remove the Neo cassette, F1 offspring were then crossed with mice harboring a cre transgene under the control of the adenovirus EIIa promoter that targets the expression of cre recombinase to the early mouse embryo (Jackson Laboratory). Our studies utilized mice in which the Neo cassette has been removed, thereby eliminating the possibility of Neo gene expression interfering with our observed results. To identify animals with the transmitted allele, tail DNA was extracted and digested with NheI and hybridized with a 3' probe. As shown in Figure 2.6A, DNA from wild-type animals contains a single fragment at $11.1 \mathrm{~kb}$, whereas heterozygous animals contain two fragments, an $11.1 \mathrm{~kb}$ wild-type fragment and a $9.2 \mathrm{~kb}$ knock-in 
A.

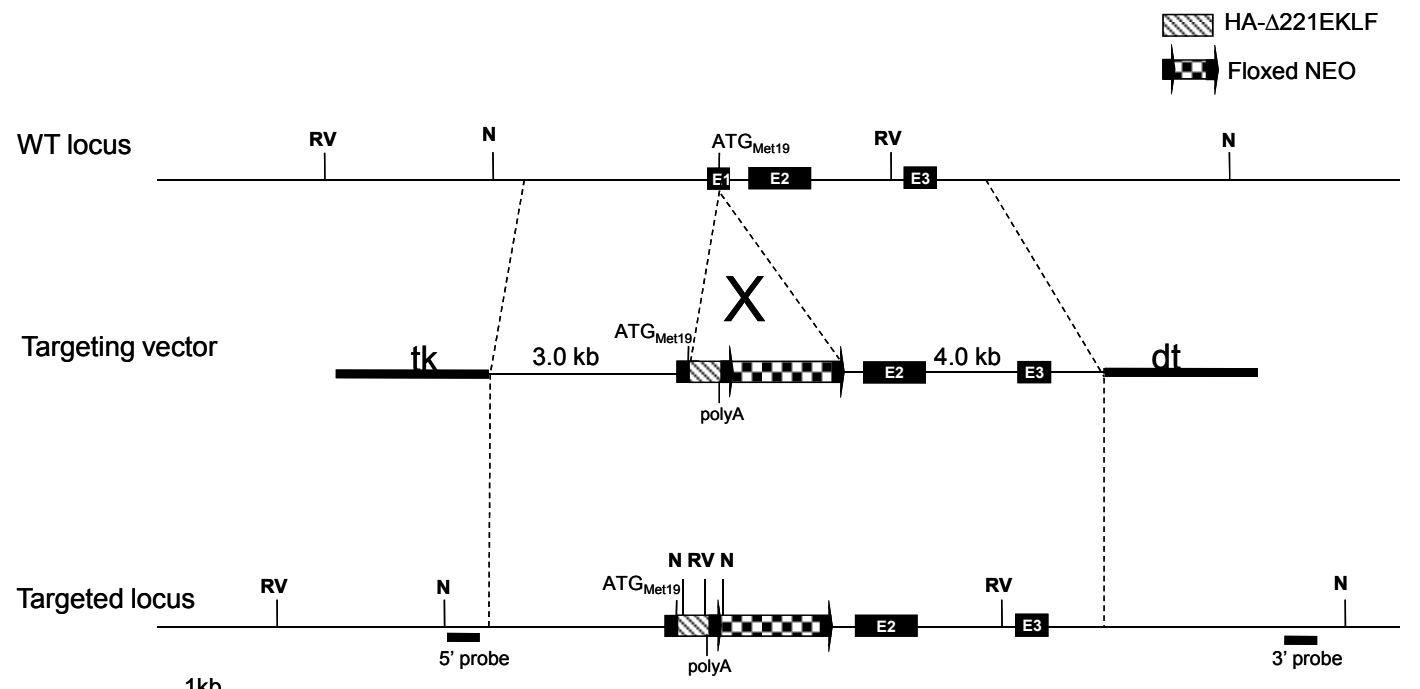

B.

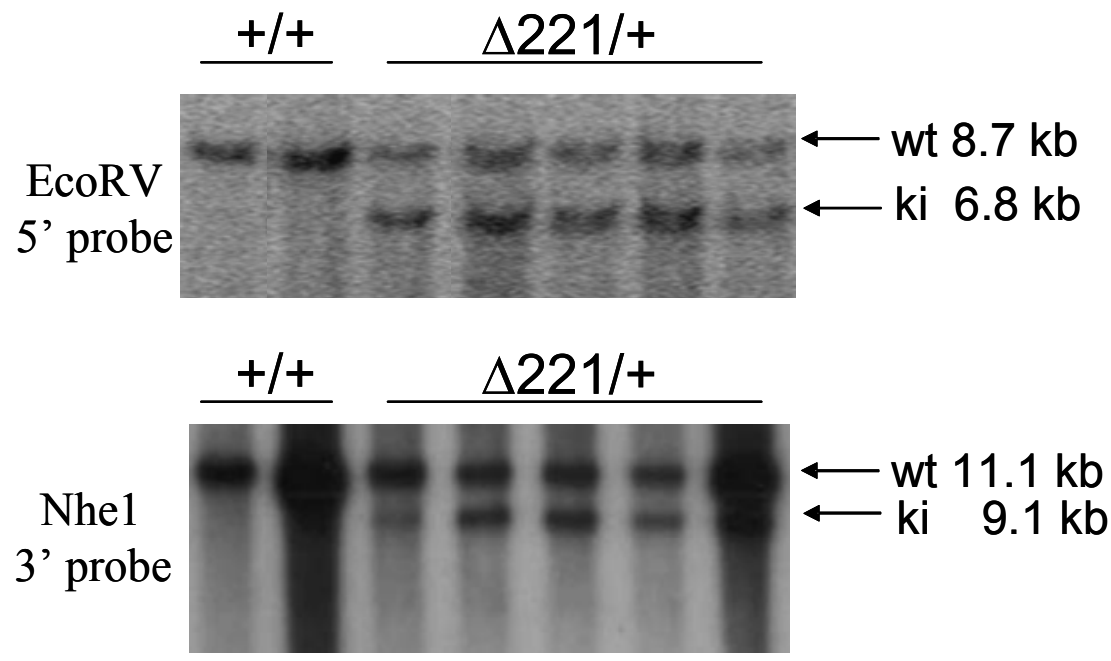

Figure 2.5. Targeting of cDNA encoding HA- $\triangle 221 \mathrm{EKLF}$ into the endogenous EKLF locus. (A) Targeting strategy showing the wild-type EKLF locus, the targeting vector used for gene targeting in ES cells, and the targeted locus in which HA- $\Delta 221 \mathrm{EKLF}$ was inserted utilizing the endogenous ATG start site thereby placing the knock-in mutant gene under the control of the cis-regulatory elements. Shaded rectangles indicate the location of the external probes used for Southern blotting. $\mathrm{N}=$ NheI, RV $=$ EcoRV, tk $=$ thymidine kinase; $\mathrm{dt}=$ diphtheria toxin (B) Southern blot analysis of ES clones using EcoRV digest with 5' probe and NheI digest and 3' probe. The wild-type allele is detected as an $8.7 \mathrm{~kb}$ and an $11.1 \mathrm{~kb}$ band, respectively. The knock-in allele is detected as a $6.8 \mathrm{~kb}$ and $9.1 \mathrm{~kb}$ band, respectively. 
A.

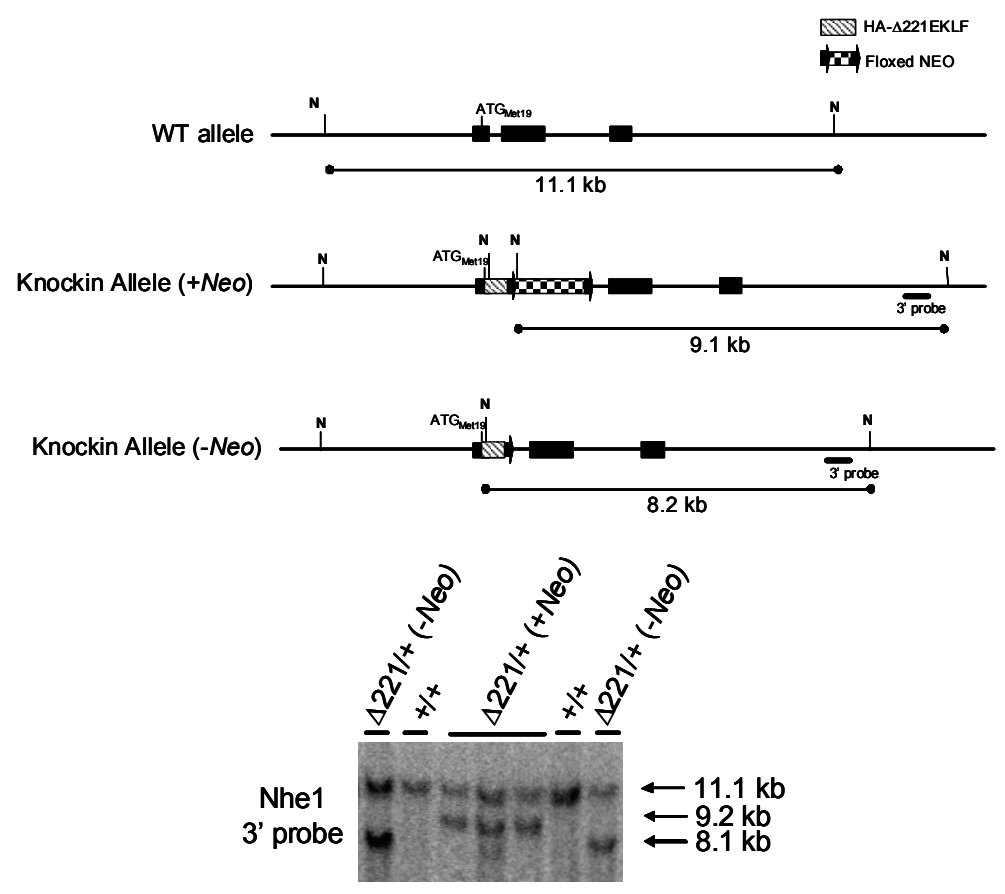

B.
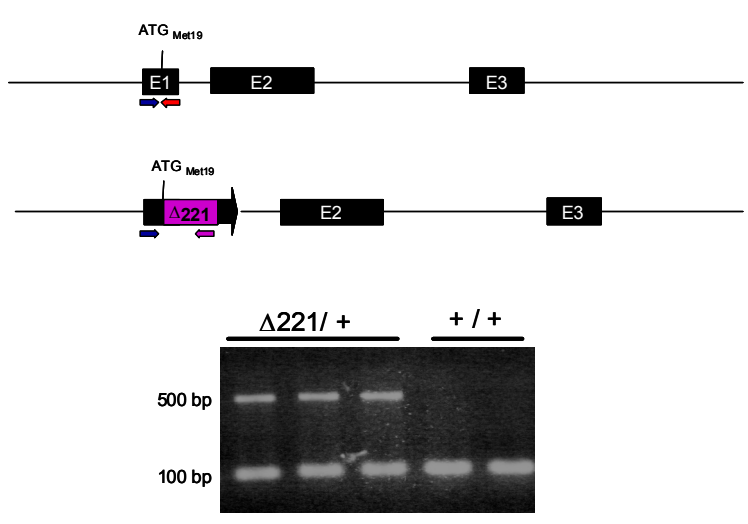

Figure 2.6. Identification of heterozygous mice expressing HA- $\triangle 221 \mathrm{EKLF}$. (A) Top panel: A schematic diagram of the wild-type allele and knock-in allele with and without Neo cassette with the predicted fragment s shown. Bottom panel: Southern blot analysis of mouse tail DNA digested with NheI and hybridized with 3' probe. The wild-type band is $11.1 \mathrm{~kb}$; knock-in bands $9.2 \mathrm{~kb}$ (with $\mathrm{Neo}$ ) and $8.1 \mathrm{~kb}$ (without $\mathrm{Neo}$ ). (B) Top panel: A diagram of the wild-type and knock-in alleles. Colored arrows indicate the location of primers used in multiplex PCR. Dark blue $=$ common forward primer; red $=$ wild-type reverse primer; purple $=$ knock-in reverse primer. Bottom panel: A representative photograph of agarose gel electrophoresis. PCR products amplified with DNA from wild-type animals and heterozygous knock-in animals. The wild-type product is $96 \mathrm{bp}$ and the knock-in product is $500 \mathrm{bp}$. 
fragment. Mice in which the Neo cassette has been excised have an $8.1 \mathrm{~kb}$ fragment, due to loss of a NheI site. Subsequently, a multiplex PCR method utilizing a common forward primer with two different reverse primers was developed to facilitate genotyping of the knock-in animals (Figure 2.6B). PCR of DNA from a wild-type animal amplified only a 96 bp fragment with the primers, whereas DNA from a heterozygous animal amplified a wild-type fragment of $96 \mathrm{bp}$ and a knock-in fragment of $500 \mathrm{bp}$ with the same primers.

Animals heterozygous for the EKLF gene have no discernable phenotype (Nuez et al., 1995; Perkins et al., 1995). There are, however, exceptions where deletion of a single gene copy leads to an abnormal phenotype (Johnson et al., 1988; Muroya et al., 2001; Deutschbauer et al., 2005) whereby the heterozygous mutation on one allele blocks the activity of the wild-type protein is still encoded by the normal allele. To determine whether the knock-in mutant protein encoding the $\triangle 221 \mathrm{EKLF}$ domain resulted in an unexpected phenotype, I evaluated $\Delta 221$ EKLF heterozygous adult mice. First, analysis of the Mendelian ratio from heterozygous matings demonstrated that $\triangle 221 \mathrm{EKLF}$ heterozygous animals survive to adulthood without any visible defects (Table 2.2). As expected, these studies also revealed that $\triangle 221 \mathrm{EKLF}$ homozygous animals are not viable (see Chapter 3).

To evaluate the effects of the heterozygous knock-in allele on mouse hematopoiesis, I examined the peripheral blood obtained from $\triangle 221 \mathrm{EKLF}$ heterozygous, EKLF-null heterozygous, and wild-type mice. Complete blood count (CBC) analysis revealed no abnormalities in the hematological parameters measured, including white blood cell (WBC) number, red blood cell (RBC) number, hemoglobin (Hgb), hematocrit (Hct), and platelet (Plt) count (Table 2.3). Similarly, Wright-Giemsa staining of the peripheral blood smear demonstrated that the circulating erythrocytes in $\triangle 221 \mathrm{EKLF}$ mice are morphologically similar to those found in wild-type and EKLF-null heterozygous animals (Figure 2.7).

\section{DISCUSSION}

To generate the knock-in alleles of EKLF, I have utilized a lambda phage-based recombination method called recombineering. This method relies on homologous recombination in $E$. coli instead of the traditional subcloning method utilizing restriction enzymes and DNA ligases for vector construction, thus eliminating the time and difficulty encountered in traditional subcloning methods (Yu et al., 2000; Copeland et al., 2001; Liu et al., 2003). The basic steps of recombineering are outlined in Figure 2.1. The targeting vectors have been designed in such a way that the cDNA encoding the truncation mutation of EKLF (i.e. $\triangle 221 \mathrm{EKLF}$ ) would be expressed under the endogenous EKLF promoter and cis-regulatory elements. Hence, it is anticipated that the expression of the mutant genes, $\Delta 164 \mathrm{EKLF}, \Delta 221 \mathrm{EKLF}$, and $\triangle 253 \mathrm{EKLF}$, would resemble that of the wild-type endogenous EKLF protein. This approach ensures the proper expression of the mutant factor during development and eliminates some of the obstacles in using cell lines and artificial rescue constructs (Tsai et al., 1998). This approach has been used to 
Table 2.2. Genotyping of adult animals from heterozygous matings.

Genotype Number of adult animals ${ }^{\mathrm{a}}$

WT/WT

$213 / 154$

$\Delta 221 \mathrm{EKLF} / \mathrm{WT}$

$402 / 307$

$\Delta 221 \mathrm{EKLF} / \Delta 221 \mathrm{EKLF}$

$0 / 154$

Total $^{\mathrm{b}}$

615

${ }^{a}$ numbers are expressed as observed / expected

${ }^{\mathrm{b}}$ total number of animals analyzed 
Table 2.3. Complete blood count analysis of $\triangle 221 \mathrm{EKLF}$ heterozygous adult mice.

\begin{tabular}{|c|c|c|c|c|c|}
\hline Parameter & WT/WT & WT/ $\Delta 221 E K L F$ & (p-value) & WT/EKLF ${ }^{\text {null }}$ & (p-value) \\
\hline $\mathrm{WBC}\left(\times 10^{3} / \mu \mathrm{L}\right)$ & $6.22 \pm 1.73$ & $10.67 \pm 2.48$ & $(0.54)$ & $11.40 \pm 1.94$ & $(0.13)$ \\
\hline $\mathrm{RBC}\left(\times 10^{6} / \mu \mathrm{L}\right)$ & $9.09 \pm 0.68$ & $8.48 \pm 0.51$ & $(0.49)$ & $9.10 \pm 0.34$ & $(0.74)$ \\
\hline $\mathrm{Hgb}(\mathrm{g} / \mathrm{dL})$ & $13.40 \pm 0.70$ & $12.83 \pm 0.23$ & $(0.28)$ & $13.43 \pm 0.42$ & $(0.97)$ \\
\hline Hct $(\%)$ & $39.60 \pm 1.73$ & $38.30 \pm 2.44$ & $(0.25)$ & $39.07 \pm 1.88$ & $(0.95)$ \\
\hline $\operatorname{Plt}\left(\mathrm{x} 10^{3} / \mu \mathrm{L}\right)$ & $824 \pm 148$ & $1005 \pm 73.5$ & $(0.13)$ & $1048 \pm 83$ & $(0.13)$ \\
\hline
\end{tabular}

Note: Animals were age and sex matched with 3 adult mice analyzed per cohort. Data shown are mean \pm standard deviation. Data were analyzed by the student's t-test; $p$-values are listed in parentheses compared to WT. 

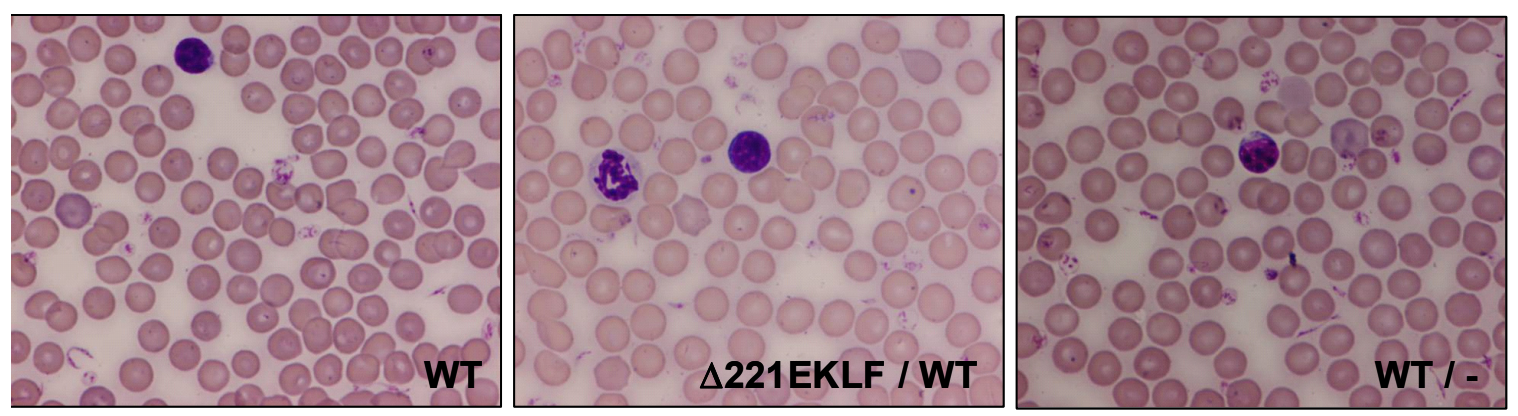

Figure 2.7. Normal circulating erythrocytes in $\triangle 221 E K L F$ heterozygous adult animals. Peripheral blood smear (Wright-Giemsa) of adult animals obtained by retroorbital bleeds. Erythrocytes from $\triangle 221 E K L F$ heterozygous animals are morphologically similar to erythrocytes from wild-type and EKLF heterozygous animals. Magnification $\mathrm{x} 100$. 
demonstrate that myogenin can replace Myf5 in rib cage development (Hanks et al., 1998) and that En-2 can replace the function of En-1 in mid-hindbrain development (Wang et al., 1996). Similarly, knock-in mutation of GATA-3 into the GATA-1 locus can partially replace the highly homologous factor in erythroid cell development (Tsai et al., 1998).

Utilizing the recombineering method, I found it difficult to co-electroporate the gap-repaired plasmid and the mini-targeting cassette as originally described (Liu et al., 2001). Instead of co-electroporation, I electroporated the mini-targeting cassette into electrocompetent EL350 cells which harbored the correct retrieval vector as determined by restriction enzyme and sequence analysis. This alternative method required an intermediate step to make the retrieval vector-containing EL350 cells electrocompetent before electroporation with the mini -targeting cassette. In contrast, the previously described co-electroporation method required a single step in which the retrieval vector and mini-targeting cassette DNA were co-electroporated into previously frozen electrocompetent EL350 bacterial cells. The time required for my altered approach was not significant and did not greatly affect the total time required to obtain the final targeting vectors.

Although the major step of vector construction relied on homologous recombination in bacteria by taking advantage of the lambda phage recombination genes, certain intermediate steps such as construction of a plasmid to retrieve an $11 \mathrm{~kb}$ of sequence spanning the EKLF gene from the EKLF BAC and the mini-targeting vectors utilized traditional subcloning methods. As a result, I encountered obstacles common to traditional subcloning. First, I found it difficult to perform a triple ligation (fragments $\mathrm{AB}, \mathrm{XY}$, and vector) to construct the retrieval vector. Very few colonies grew on the selection plates, and the selected colonies did not harbor the correct plasmid. To circumvent this problem, I increased the ratio of vector to insert in the ligation reaction from 1:3 to 1:5 which yielded more colonies to screen. Similarly, I first tried to construct the mini-targeting vector in a single reaction by ligating fragments CD, HA- $\triangle 221 \mathrm{EKLF}$, Neo-cassette, and EF into a pBlueScript vector. This yielded no growth on the agar plates. An alternative method was to sequentially subclone each fragment into a vector until a complete mini-targeting vector was constructed. To attempt this altered strategy, I had to design new PCR primers for fragments $\mathrm{CD}$ and EF with different restriction sites to facilitate subcloning. To my advantage, I used the PL450 vector which already contained the $\mathrm{NeO}$ cassette to subclone in fragment EF. Next, I performed a triple ligation using increased ratio of vector to inserts to subclone fragments CD and HA$\triangle 221 \mathrm{EKLF}$ into PL450-EF. Overall, construction of the mini-targeting vectors utilizing traditional subcloning methods proved to be the most time consuming portion of generating the knock in alleles of EKLF. However, I was not hindered by the restriction sites as the site for the restriction enzyme of interest was conveniently included in the PCR amplification primers.

In my targeting strategy, I have included a Neo cassette adjacent and downstream of the $\triangle 221 \mathrm{EKLF}$ cDNA to facilitate selection during recombineering in bacteria and targeting in ES cells. Previous studies of knock-out mice have demonstrated the 
unpredictable phenotypes that can be caused by the retention of Neo cassettes (Olson et al., 1996; Pham et al., 1996). For example, studies in which several specific mutations were generated within the human or murine $\beta$-globin LCR by insertion of PGK-Neo led to abrogation of the expression of multiple globin genes downstream from the cassette. However, deletion of the selectable marker cassette resulted in restoration of LCR activity, suggesting that the cassette interfered with normal interactions between the LCR and downstream regulatory elements in the gene promoters (Pham et al., 1996). To improve $\triangle 221 \mathrm{EKLF}$ expression that could be altered by an adjacent downstream $\mathrm{NeO}$ transgene and to rule out any toxic effects of Neo expression at this locus, the Neo cassette in $\triangle 221 \mathrm{EKLF}$ heterozygous mice was removed by breeding to a cre transgenic mouse line (Lasko et al., 1996). Subsequent studies in this dissertation have been completed in mice in which the Neo cassette has been removed.

Analysis of the $\triangle 221 \mathrm{EKLF}$ heterozygous animals provides an early glimpse into the functionality of the mutant knock-in protein in vivo. Given that adult $\Delta 221 \mathrm{EKLF}$ heterozygous animals develop normally with no discernible phenotype, I speculate that the mutant knock-in allele does not exhibit a dominant negative or haploinsufficient phenotype. This observation is supported by my results from the $\mathrm{CBC}$ analysis and peripheral blood smear which are consistent with a normal hematological phenotype. Although the results are not significant ( $p$-value $>0.05$ ), it should be noted that these studies were performed with three animals in each cohort. To minimize the possibility of prematurely rejecting a dominant negative phenotype in $\triangle 221 \mathrm{EKLF}$ animals, studies in $\triangle 221$ EKLF heterozygous animals should be repeated with a larger sample size. Indeed, it is not uncommon for this phenotype to occur with loss-of-function studies such as been described for the transcription factors TWIST (Johnson et al., 1988), GATA3 (Muroya et al., 2001), and STAT6 (Bürgis \& Gessner, 2007). For instance, mutant TWIST protein is expressed and acts as a dominant negative protein by binding to target promoters and then blocking gene transcription (Johnson et al., 1998). I will demonstrate in Chapter 3 that the mutant $\Delta 221 \mathrm{EKLF}$ protein is expressed in fetal liver and possesses chromatin remodeling function in vivo.

In summary, I have constructed knock-in targeting vectors in which cDNAs encoding three different amino terminal truncation mutants of EKLF have been engineered into the initiation site of the endogenous murine EKLF gene utilizing the recombineering method. Using this method, I have constructed all three targeting vectors in a relatively short time. Subsequently, the vectors have been used to target the endogenous EKLF locus in ES cells via homologous recombination in order to generate $\triangle 221$ EKLF heterozygous animals. Following the recommendations of my research committee, I have chosen to focus my dissertation studies on one strain of mice, the $\triangle 221$ EKLF expressing animals. This domain of EKLF appears to be the most interesting to study in vivo as it has been previously identified in cellular assays to possess chromatin remodeling properties, however, lacks the ability to transactivate $\beta$-globin gene transcription (Brown et al., 2002). This animal model will be useful to test my hypothesis that expression of $\triangle 221 \mathrm{EKLF}$ domain is sufficient to reorganize the local chromatin architecture at the $\beta$-globin promoter but is insufficient to transcriptionally activate $\beta$ globin in vivo. In the following chapter, I will describe the functional consequences of 
expression of $\triangle 221$ EKLF mutant protein in mice homozygous for the knock-in locus. 


\section{CHAPTER 3: CHARACTERIZATION OF THE MOLECULAR CONSEQUENCES OF $\triangle 221 E K L F$ EXPRESSION IN VIVO}

\section{INTRODUCTION}

The $\beta$-globin locus serves as a paradigm for studying the regulation of a multigene locus. The murine $\beta$-globin locus, located on chromosome 7 , encodes four genes $\left(5^{\prime}-\varepsilon y-\beta h 1-\beta^{\text {maj }}-\beta^{\text {min }}-3\right.$ ') which are arranged in the order of their expression during ontogeny. Appropriate regulation of the globin genes in a tissue- and developmentalspecific manner resides in DNA elements located proximal and distal to the genes. The $\beta$-globin locus control region (LCR) is a powerful DNA element located far upstream of the globin genes. The LCR is required for high level expression of all the genes during development (Grosveld et al., 1987; Bender et al., 2000). The precise mechanism of how the LCR enhances globin gene transcription is still unsettled; however, it is thought to involve some method of physical communication between the LCR and the globin genes (Bulger \& Groudine, 1999; Engel \& Tanimoto, 2000).

The murine LCR consists of a series of DNase I hypersensitive sites (HS1-6) that contain binding sites for transcriptional factors. A current hypothesis regards the LCR as a holocomplex that enhances globin gene expression through recruitment of chromatin remodeling, coactivator, and transcriptional complexes (Wijgerde et al., 1995; Bungert et al., 1999; Tolhuis et al., 2002). Indeed, it has been shown that RNA polymerase II (PolII) is recruited to the LCR (Johnson et al., 2003). Similarly, GATA-1, NF-E2, and EKLF have been shown to bind the HS of the LCR (Pevny et al., 1995; Fujiwara et al., 1996; Forsberg et al., 2000; Zhou et al., 2006). Furthermore, both GATA-1 and EKLF are required for $\beta$-globin LCR/promoter interaction (Drissen et al., 2004; Vakoc et al., 2005).

The $\beta$-globin promoter contains several cis-acting elements that modulate the expression of the $\beta$-globin gene. Of these, the CACC motif is of the most interest to the present study. Mutations of this consensus sequence lead to a thalassemic state (Orkin et al., 1984) and specifically disrupt the binding of EKLF (Miller \& Bieker, 1993; Feng et al., 1994). Disruption of the EKLF gene by homologous recombination has demonstrated its non-redundant role during erythroid development. EKLF-null embryos die of a lethal anemia by embryonic day 14-15 of gestation (E14-15), as definitive erythroid cells failed to produce $\beta$-globin transcripts in vivo (Nuez et al., 1995; Perkins et al., 1995). Further analysis of these EKLF null embryos revealed loss of a developmentally specific DNase I hypersensitivity site in the proximal $\beta$-globin promoter of E14 fetal liver erythroblasts derived from EKLF null embryos (Wijgerde et al., 1996). Because the degree of DNase I hypersensitivity of a given locus correlates with nucleosomal remodeling (Steger \& Workmann, 1996; Pazin et al., 1997), these findings are consistent with the idea that EKLF is required for chromatin reorganization at the $\beta$ globin promoter in definitive erythroid cells.

Studies to elucidate the molecular mechanisms by which EKLF modulates 
chromatin remodeling at the $\beta$-globin promoter have relied heavily on in vitro chromatin reconstitution assays (Armstrong et al., 1998; Kadam et al., 2000). These studies have identified a SWI/SNF-containing multiprotein complex, E-RC1, which interacts with the zinc finger DNA binding domain of EKLF. This interaction results in chromatin remodeling and transcriptional activation of a chromatinized $\beta$-globin template.

Additional evidence supporting this interaction comes from studies demonstrating that EKLF interacts with BRG1, a subunit of E-RC1 (Zhang et al., 2001). Contrary to in vitro chromatin assays (Kadam et al., 2000), our laboratory has shown that the zinc finger domain alone is not sufficient to remodel chromatin at the $\beta$-globin promoter in erythroid cell lines (Brown et al., 2002). Additional sequences in the amino terminus are required for EKLF-dependent chromatin remodeling and EKLF interaction with BRG1. However, evidence for a direct interaction between EKLF and SWI/SNF components in vivo is still lacking.

To test my hypothesis that expression of $\triangle 221 \mathrm{EKLF}$ alone is sufficient to alter the local chromatin structure at the $\beta$-globin locus in vivo, I will explore the consequences of expression of $\triangle 221 \mathrm{EKLF}$ and analyze the determinants required for chromatin remodeling and $\beta$-globin transcription in vivo. Despite the advances in our understanding of the function of EKLF as a chromatin modifier and transcriptional activator, knowledge about the direct interaction of EKLF and SWI/SNF components and how EKLF modulates chromatin in vivo remains elusive. Utilizing the newly generated $\triangle 221 \mathrm{EKLF}$ animals, I show that expression of $\triangle 221 \mathrm{EKLF}$ results in embryonic lethality similar to mice lacking EKLF. In stark contrast to lack of EKLF, expression of $\triangle 221 \mathrm{EKLF}$ completely restores the local chromatin structure at the $\beta$-globin promoter. However, this domain is unable to rescue $\beta$-globin gene transcription in vivo and fails to recruit GATA1 and NF-E2 to the $\beta$-globin promoter. My results suggest that the $\Delta 221 \mathrm{EKLF}$ domain is sufficient for chromatin reorganization at the $\beta$-globin promoter, thus priming the $\beta$ globin gene for transcriptional activation. Furthermore, I show that nucleosomal remodeling and histone modifications at the $\beta$-globin promoter are insufficient to activate high-level $\beta$-globin transcription in vivo.

\section{MATERIALS AND METHODS}

\section{RNA analysis}

Total RNA isolated from fetal liver of E14.5 embryos was subjected to RT-PCR analysis. RNA was extracted using the Trizol method and cDNA was prepared using the Superscript III First Strand System (Invitrogen) following the manufacturer's protocols. Briefly, fetal livers from E14.5 embryos were homogenized in Trizol (200-500 $\mu \mathrm{L})$ reagent (Invitrogen). RNA $(10 \mu \mathrm{g})$ was treated with Turbo DNase $(2 \mu \mathrm{L})$ (Ambion) in a $50 \mu \mathrm{L}$ reaction to remove genomic DNA contamination. Following DNase treatment, DNase-treated RNA $(11 \mu \mathrm{L})$ was used for first strand DNA synthesis. 
For RT-PCR of EKLF gene expression, cDNA $(2 \mu \mathrm{L})$ was analyzed on a BIORAD PCR machine with the following settings: $94^{\circ} \mathrm{C}$ for $3 \mathrm{~min}$, then 30 cycles of $94^{\circ} \mathrm{C}$ for $30 \mathrm{sec}, 60^{\circ} \mathrm{C}$ for $30 \mathrm{sec}, 72^{\circ} \mathrm{C}$ for $1 \mathrm{~min}$, followed by $72^{\circ} \mathrm{C}$ for $10 \mathrm{~min}$. PCR products were analyzed on agarose gels. RT-PCR primers were generated using the Primer 3 program and are as follows: Exon 1: forward: 5'ATGAGGCAGAAGAGAGAGAGGAG-3'; reverse: 5'TGAGTGTACTGATGGAGGGTAAGA -3'; Exon 2: forward: 5'CACAGTACCAAGGCCACTTC -3'; reverse: 5'-GTCCCAGGTCCAAGACAATTC-5'; Exons 2/3: forward: 5'-GGGAAGAGCTACACCAAGAGC-3'; reverse: 5'GAAGGGACGATGTCCAGTGT-3'; HA- 221 EKLF: forward: 5'TATGCTAGCCTCCCGGGTTA-3'; reverse: 5'-GGTCTCGGCTATCACACCTG-3'; HPRT: forward: 5'- GCAGTACAGCCCCAAAATGG-3'; reverse: 5'AACAAAGTCTGGCCTGTATCCAA-3' (Hodge et al., 2006).

$\beta$-globin mRNA and primary transcripts were measured by real time RT-PCR utilizing SYBR green fluorescence on an Applied Biosystems Prism 7000 machine. The amount of product was determined relative to a standard curve generated from a titration of the cDNA. Dissociation curves after amplification showed that primer pairs generated single products. The primers used for real time RT-PCR are shown in Appendix Table A.5. Each sample was run in duplicate with at least five independent experiments performed.

\section{Protein analysis}

Whole cell extracts were prepared from fetal liver of E14.5 embryos using the PTER reagent (Pierce) according to the manufacturer's recommendations. Protein concentration was measured by the Bradford Assay reagent (BioRad). One hundred micrograms of protein was mixed with loading dye and denatured by boiling for $5 \mathrm{~min}$. The proteins were resolved on a $15 \%$ SDS-PAGE gel followed by wet transfer. The blots were blocked in $5 \%$ milk in $0.1 \% \mathrm{PBS} /$ Tween- 20 for $1 \mathrm{~h}$ at room temperature with constant rocking. Primary antibody at 1:200 dilution or 1:400 dilution for HA and EKLF, respectively, was added and the blots were probed overnight in the cold room with constant rocking. Blots were washed once for $15 \mathrm{~min}$ and twice for $5 \mathrm{~min}$ times with $0.1 \% \mathrm{PBS} /$ Tween-20. Secondary antibody conjugated with horseradish peroxidase (HRP) was added at a dilution of 1:1000 and incubated for $1 \mathrm{~h}$ at room temperature. The blots were washed three times as before with $0.1 \%$ PBS/Tween-20. Peroxidase activity was visualized by enhanced chemiluminescence (ECL) using Western blotting detection reagents from Amersham Biosciences. The Western blots were probed with HAantibody (Santa Cruz), Cunningham laboratory generated EKLF-antibody directed toward the zinc fingers (J.M.C unpublished), and actin-HRP (Abcam) as loading control. 


\section{Cytospin, sections, and staining}

Fetal liver erythroblasts from E14.5 embryos were made into single cell suspension in D10 (DMEM + 10\% FBS) and cytocentrifuged onto glass slides. For immuno-localization studies, the cells were fixed in 4\% formaldehyde for $20 \mathrm{~min}$, permeabilized with $0.1 \%$ Triton X-100-PBS, and blocked in $10 \%$ normal donkey serum (Jackson ImmunoResearch) for $1 \mathrm{hr}$ at room temperature. Anti-HA antibody was added to the slides and incubated at $4^{\circ} \mathrm{C}$ overnight. A secondary antibody, conjugated to FITC (Molecular Probes), was applied for another $2 \mathrm{~h}$ of incubation at room temperature. DNA staining was carried out using DAPI (4', 6'diamidino-2-phenylindole) (Vector Shield). Slides were visualized with a Leica DM IRB fluorescence microscope.

Staining with benzidine-Giemsa (Sigma) was performed according to the manufacturer's recommendations. Briefly, cytospin preparations were fixed in cold methanol for $2 \mathrm{~min}$, allowed to air dry, and then stained in benzidine for $20 \mathrm{~min}$. After $20 \mathrm{~min}$, the slides were rinsed with distilled water and placed in Giemsa stain for another $20 \mathrm{~min}$. Slides were rinsed with water, air dried, and mounted with cover slips.

To prepare sections of the fetal liver, wild-type, $\triangle 221 \mathrm{EKLF}$, and EKLF-null E14.5 fetal livers were immersion fixed in 4\% formalin overnight, or longer, before submission to the Core Pathology Department at the University of Chicago. The fetal livers were processed for sectioning and stained with H\&E and Prussian blue. Slides were viewed under a Nikon Eclipse E400 microscope fitted with a camera.

\section{DNase I sensitivity assays}

DNase I sensitivity assays were performed essentially as described by McArthur et al. (2001) with slight modifications. Briefly, fetal livers were harvested from E14.5 mouse embryos and made into single cell suspension by passing through a $70 \mu \mathrm{m}$ cell strainer. Cells $\left(6 \times 10^{6}\right)$ were washed once with cold PBS and resuspended with $5 \mathrm{ml}$ of buffer A (15 mM Tris $\mathrm{HCl}$ (pH 7.6), $60 \mathrm{mM} \mathrm{KCl,} 15 \mathrm{mM} \mathrm{NaCl}, 1 \mathrm{mM}$ EDTA, $0.5 \mathrm{mM}$ EGTA, $0.5 \mathrm{mM}$ spermidine, $0.15 \mathrm{mM}$ sperminine). Cells were lysed in the presence of $0.5 \%(\mathrm{v} / \mathrm{v}) \mathrm{NP} 40$, and nuclei were collected and resuspended in $1.2 \mathrm{~mL}$ of digestion buffer (buffer A supplemented with $3 \mathrm{mM} \mathrm{CaCl}_{2}, 75 \mathrm{mM} \mathrm{NaCl}$ ). $200 \mu \mathrm{L}$ of the nuclei mixture was added to Eppendorf tubes containing $50 \mu \mathrm{L}$ of the digestion mixture containing 0-18 units of DNase I (ROCHE). Digestions were carried out at $37^{\circ} \mathrm{C}$ for five minutes before being stopped by the addition of an equal volume of stop buffer $(0.1 \mathrm{M}$ $\mathrm{NaCl}, 0.1 \%$ (w/v) SDS, $50 \mathrm{mM}$ Tris- $\mathrm{HCl}$ (pH 8.0), $100 \mathrm{mM}$ EDTA). The samples were treated with proteinase $\mathrm{K}(500 \mu \mathrm{g} / \mathrm{mL})$ at $55^{\circ} \mathrm{C}$ overnight and DNA recovered by extraction with phenol/chloroform and precipitation in ethanol. The DNA was then dialyzed against two changes of TE buffer, re-precipitated and diluted in water to a concentration of $10 \mathrm{ng} / \mu \mathrm{L}$ as measured by spectrophotometry.

Real time PCR was performed on the DNA samples using SYBR green mix in a total reaction volume of $25 \mu \mathrm{L}$. To quantify the amount of DNA remaining following 
DNase I treatment, a standard curve was generated from titration of undigested genomic DNA. The values at each concentration of DNase I was normalized to a previously determined DNase I insensitive gene, Nf-M (McArthur et al., 2001). Dissociation curves after amplification showed that a single product was formed for each primer pair. The primer pairs used were: $\beta$ maj promoter forward: 5'-

CAGGGAGAAATATGCTTGTCATCA-3' and $\beta$ maj promoter reverse:

5'-GTGAGCAGATTGGCCCTTACC-3'; Nf-M forward: 5'-

GCTGGGTGATGCTTACGACC-3' and Nf-M reverse: $5^{\prime}-$

GCGGCATTTGAACCCTCTT-3' (McArthur et al., 2001).

\section{Chromatin immunoprecipitation assays}

ChIP assays were performed as previously described (Forsberg et al., 2000; Kiekhaefer et al., 2002), with slight modifications. Fetal livers from E13.5 mouse embryos were harvested and passed separately through a $70 \mu \mathrm{m}$ cell strainer into $20 \mathrm{ml}$ of DMEM-based medium supplemented with $10 \%$ FBS. Protein-DNA cross-linking was performed by incubating suspensions of fetal liver (at a concentration of $10^{6}$ cells $/ \mathrm{mL}$ ) with formaldehyde at a final concentration of $0.8 \%$ for $10 \mathrm{~min}$ at room temperature with gentle agitation. Glycine $(0.125 \mathrm{M})$ was added to quench the reaction. Cells were then collected by centrifugation at $1000 \mathrm{rpm}$ for $5 \mathrm{~min}$, washed in cold PBS and a second wash in cold PBS supplemented with Roche complete EDTA-free protease inhibitor and PMSF (Fluka). Cross-linked cells were snap frozen and stored at $-70^{\circ} \mathrm{C}$ until further processing.

Immunoprecipitation was performed with $1 \times 10^{7}$ cells per IP condition. The buffer composition used in the ChIP assay is listed in the Appendix Table A.3. Nuclei were isolated by incubation in cell lysis buffer for $10 \mathrm{~min}$ on ice followed by centrifugation at $600 \times g$ for $5 \mathrm{~min}$. Nuclei were lysed in nuclei lysis buffer for $10 \mathrm{~min}$ on ice. The lysate was sonicated for 20 minutes total using the Bioruptor sonicator (Diagenode, Belgium) set on High power and cycles of 30 seconds $\mathrm{ON}$ and 30 seconds OFF to obtain an average size of $500 \mathrm{bp}$. Soluble chromatin was diluted 1:4 with IP dilution buffer and precleared by addition of $5 \mu \mathrm{L} / \mathrm{mL}$ preimmune serum followed by 50 $\mu \mathrm{L}$ of salmon sperm DNA/Protein A agarose (Millipore). An aliquot $\left(1 / 10^{\text {th }}\right.$ of the total volume) of precleared chromatin was removed (input) and used in the subsequent PCR analysis. The remainder of the chromatin was incubated with and without primary antibodies for 4 hours at $4^{\circ} \mathrm{C}$. Immune complexes were collected by incubation with 75 $\mu \mathrm{L}$ salmon sperm DNA/Protein A agarose (Millipore) for $2 \mathrm{~h}$ at $4^{\circ} \mathrm{C}$. A control sample was prepared in all experiments in which IP wash buffer 1 was added instead of chromatin. Protein A agarose pellets were washed twice with $500 \mu \mathrm{L}$ aliquots of IP wash buffer 1, once with IP wash buffer 2, and twice with TE buffer. Immune complexes were eluted twice with $100 \mu \mathrm{L}$ of IP elution buffer made fresh and $\mathrm{NaCl}(0.4 \mathrm{M})$ were added, and cross-links were reversed by incubation overnight at $65^{\circ} \mathrm{C}$. Samples were digested with Proteinase $\mathrm{K}(0.3 \mathrm{mg} / \mathrm{mL})$ for $2 \mathrm{~h}$ at $45^{\circ} \mathrm{C}$. DNA was purified by Qiaquick PCR purification kit (QIAGEN) and eluted twice with $50 \mu \mathrm{L}$ elution buffer each time.

Immunoprecipitated DNA $(2 \mu \mathrm{L})$ was analyzed by real-time PCR (Applied 
Biosystems Prism 7000) with the appropriate primer pairs. Samples from at least three independent immunoprecipitations were analyzed. Product was quantified by SYBR green fluorescence in $25 \mu \mathrm{L}$ reactions, and the amount of product was determined relative to a standard curve generated from a titration of input chromatin. Dissociation curves after amplification showed that primer pairs generated single products.

Primer pairs used in ChIP analysis are listed in Appendix Table A.4. The antibodies used in ChIP analysis were anti-HA antibody (Santa Cruz, sc-805x), antiacetylated histone H3 antibody (Upstate, 06-599), anti-RNA PolII (Santa Cruz, sc-899x), anti-GATA-1 (Abcam, ab11963 ), and anti-NF-E2 (Santa Cruz, sc-291x ). Normal rabbit IgG (Santa Cruz, sc-2027) was used as a control.

\section{RESULTS}

\section{$\Delta 221$ EKLF homozygous animals die in utero and exhibit ineffective erythropoiesis}

Initial studies analyzing the matings of $\triangle 221 \mathrm{EKLF}$ heterozygous animals (described in Chapter 2) revealed that $\triangle 221 \mathrm{EKLF}$ homozygous adult mice are not viable. Of 615 adult mice genotyped, no live born $\Delta 221$ EKLF homozygous animals were observed. This observation is consistent with an embryonic lethal phenotype.

To determine when the animals succumb in utero, timed pregnancies were established. These studies revealed that $\triangle 221 \mathrm{EKLF}$ homozygous animals died by day 15.5 (E15.5) of gestation, a similar timepoint to EKLF-null embryos (Nuez et al., 1995; Perkins et al., 1995) (Table 3.1). Furthermore, gross examination of the $\Delta 221 \mathrm{EKLF}$ embryos at day 15.5 of gestation revealed a pale embryo with a smaller and paler fetal liver as compared to a wild-type littermate control (Figure 3.1). These observations are consistent with the phenotype previously described in EKLF-null embryos of similar chronology (Perkins et al., 1995).

The $\Delta 221 E K L F$ embryos appeared to lack any blood in the circulatory system, consistent with defective erythropoiesis in vivo. This observation was supported by benzidine-Giemsa staining of cytospun preparations of fetal liver erythroblasts and Prussian blue staining of fetal liver sections. As shown in Figure 3.2, there are very few benzidine-positive cells in fetal liver from $\triangle 221$ EKLF embryo. To quantify the difference, the number of benzidine-positive cells was manually counted under low magnification. As shown in Table 3.2, similar to EKLF-null embryos, $\triangle 221 \mathrm{EKLF}$ embryos had 33\% fewer benzidine-positive cells when compared to wild-type embryos. Benzidine stains for hemoglobin (i.e. brown color), a hallmark of terminal erythroid differentiation (Zhang et al., 2003). These observations, thus, suggest a failure of the $\triangle 221 \mathrm{EKLF}$-expressing erythroblasts to properly accumulate hemoglobin and execute terminal erythroid differentiation as previously demonstrated in EKLF-null cells (Drissen

et al., 2005; Pilon et al., 2008). Furthermore, the $\Delta 221 \mathrm{EKLF}$ erythroblasts, like EKLFnull erythroblasts, are misshapen as evident by the irregularly shaped cell membrane 
Table 3.1. Genotyping of embryos at different stages of gestation from heterozygous matings.

\begin{tabular}{lccccc}
\hline Genotype & E10.5 $^{\text {a }}$ & E13.5 & E14.5 & E15.5 & Adult \\
\hline WT/WT & $12 / 12$ & $30 / 27$ & $109 / 89$ & $17 / 29$ & $213 / 154$ \\
$\Delta 221 \mathrm{EKLF} / \mathrm{WT}$ & $22 / 23$ & $57 / 55$ & $175 / 179$ & $29 / 27$ & $402 / 307$ \\
$\Delta 221 \mathrm{EKLF} / \Delta 221 \mathrm{EKLF}$ & $13 / 12$ & $22 / 27$ & $73 / 89$ & $4 / 13$ & $0 / 154$ \\
Total $^{\text {b }}$ & 47 & 109 & 357 & 53 & 615 \\
\hline
\end{tabular}

${ }^{\mathrm{a}}$ numbers are expressed as observed/expected

${ }^{b}$ total number of animals analyzed 

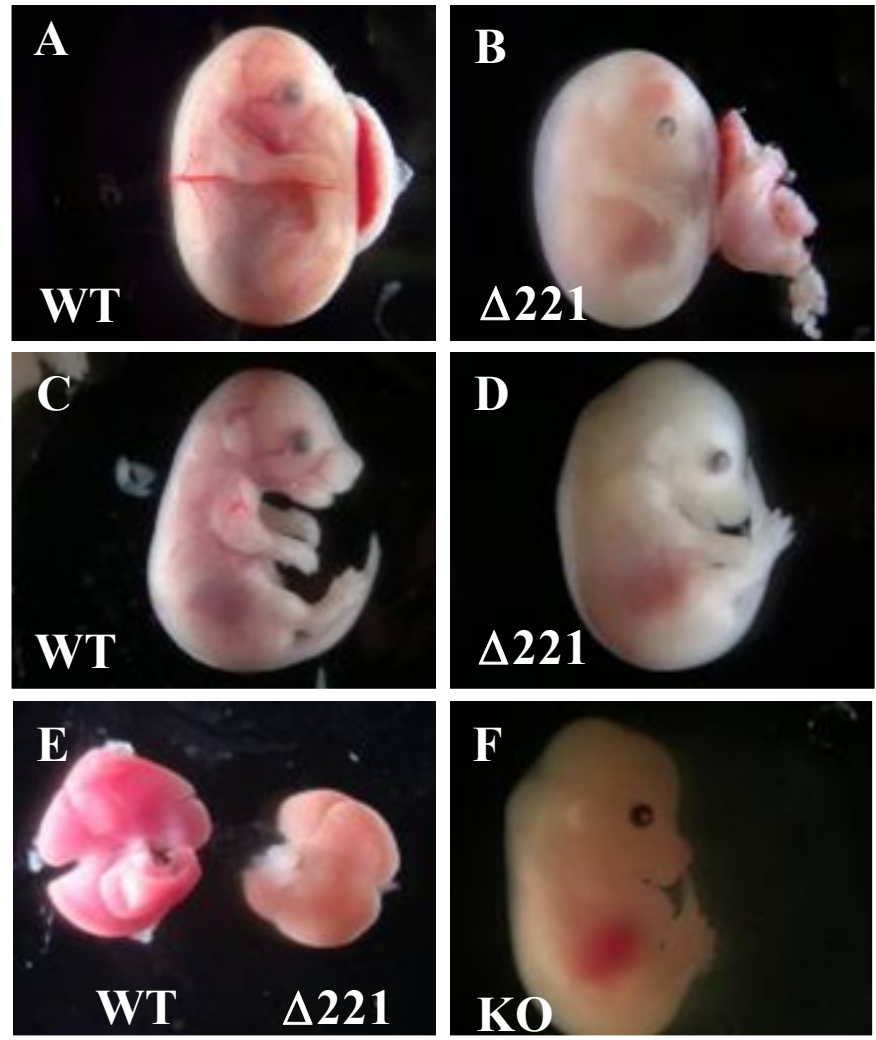

Figure 3.1. $\triangle 221 E K L F$ embryos are anemic. (A-D) Photographs of wild-type and $\triangle 221 \mathrm{EKLF}$ embryos at E15.5. The homozygous $\Delta 221 \mathrm{EKLF}$ embryo is pale and slightly smaller compared to wild-type littermate. A marked deficit of circulating red cells is evident. (E) Fetal liver from E15.5 wild-type and $\Delta 221 \mathrm{EKLF}$ embryos. The $\Delta 221 \mathrm{EKLF}$ fetal liver is smaller and paler than fetal liver from a littermate control. (F) Photograph of E15.5 EKLF-null embryo. The EKLF-null embryo is pale. 

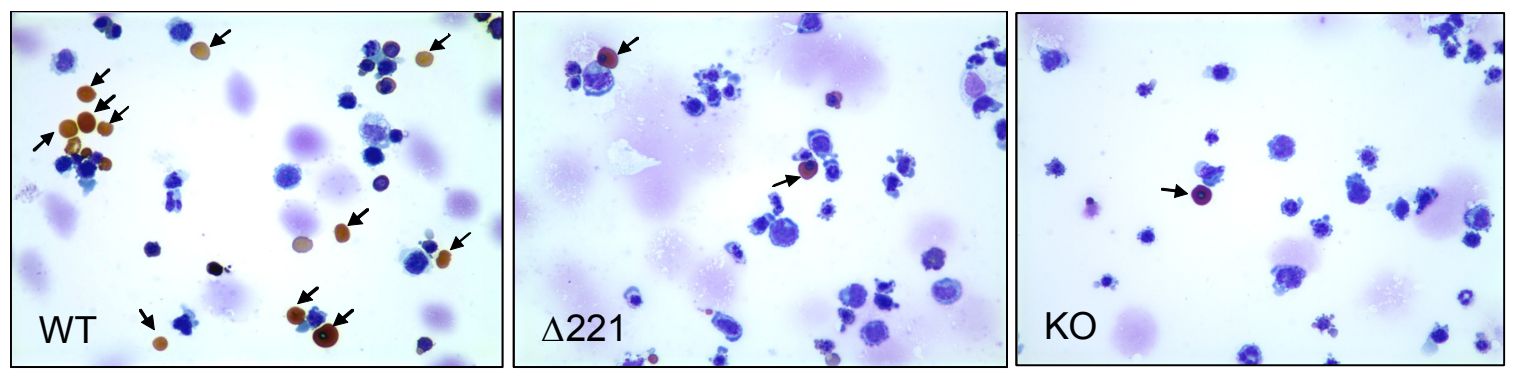

Figure 3.2. Defects in cell shape and content in $\triangle 221 \mathrm{EKLF}$ fetal liver erythroblasts. Cytospin preparations of E14.5 fetal liver erythroid cells were stained with benzidineGiemsa. There are fewer benzidine-positive cells (denoted by arrowheads) from $\triangle 221 E K L F$ fetal liver. Defective erythroid cell membrane is noted in $\Delta 221 E K L F$. Original magnification x 100 .

Table 3.2. Quantitative analysis of benzidine-positive E14.5 fetal liver erythroblasts.

Genotype \# benzidine-positive cells $/ 0.24 \mathrm{~mm}^{2}$ field

WT $25 \pm 4.2$

$\Delta 221 \mathrm{EKLF} / \Delta 221 \mathrm{EKLF}$

$8 \pm 2.7$

$\mathrm{KO}$

$7 \pm 1.3$

Note: Data shown as average number of benzidine-positive cells counted from three different fields $( \pm S D)$. Fetal liver from 2-3 embryos of each genotype were analyzed. 
compared to wild-type erythroblasts. This observation is consistent with the idea that $\triangle 221 E K L F$ erythroblasts have membrane defects as has been described in EKLF-null cells (Drissen et al, 2005; Hodge et al., 2006). Similarly, Prussian blue staining of fetal liver sections revealed excess iron deposits (i.e. blue particles) in the fetal liver of $\Delta 221$ EKLF embryos (Figure 3.3, bottom panel). This observation is consistent with the idea that $\triangle 221 \mathrm{EKLF}$ expressing erythroblasts fail to properly accumulate hemoglobin during terminal erythroid differentiation similar to that described in erythroblasts lacking EKLF (Perkins et al., 1995; Drissen et al., 2005). Together, these observations support the idea that $\triangle 221 \mathrm{EKLF}$ mice exhibit a phenotype consistent with ineffective erythropoiesis, comparable to EKLF-null mice (Nuez et al., 1995; Perkins et al., 1995).

\section{$\triangle 221 E K L F$ is expressed in fetal liver}

To evaluate the expression of the mutant knock-in allele in vivo, RNA from fetal liver erythroblasts derived from E14.5 embryos was analyzed by RT-PCR. As shown in Figure 3.4A, the mutant transcript is detected only in RNA extracted from $\triangle 221 \mathrm{EKLF}$ fetal liver erythroblasts. Furthermore, the downstream endogenous exons 2 and 3 of EKLF, which were left intact in the targeting strategy, are not transcribed in these mice. This observation is expected given that a polyadenylation signal was included in the targeting strategy to prevent interference from the endogenous downstream exons. Thus, it appears that the mutant knock-in allele is indeed transcribed in vivo.

Because mRNA expression does not necessarily correlate with protein abundance (Greenbaum et al., 2003), Western blotting analysis was performed to detect the $\triangle 221 \mathrm{EKLF}$ mutant protein in vivo. The $\Delta 221 \mathrm{EKLF}$ encoding cDNA included a HA epitope tag at the amino terminus to facilitate immunological detection. Using HAspecific anti-sera, the mutant protein was detected in the fetal liver (Figure 3.4B). However, the results do not compare the level of mutant protein expression to that of wild-type EKLF protein. Anti-EKLF antibodies are commercially available, however, the epitopes are directed to sequences outside of the $\triangle 221 E K L F$ domain. Previously, our laboratory had generated an antibody directed against the zinc finger DNA-binding domain of EKLF (J.M.C. unpublished). Using this antibody, the mutant $\triangle 221 \mathrm{EKLF}$ protein was detected in the fetal liver of $\triangle 221 \mathrm{EKLF}$ heterozygous and homozygous embryos (Figure 3.4C) albeit at a lower level than wild-type EKLF protein. The lower level of knock-in protein could result from instability or altered regulation of the knockin protein. However, I have shown that $\triangle 221 \mathrm{EKLF}$ protein retains full chromatin remodeling properties in a similar manner to wild-type EKLF protein (see below). More importantly, the endogenous wild-type EKLF protein is not detected in the fetal liver of homozygous knock-in animals. The subcellular localization of the mutant protein was analyzed by immuno-staining. As shown in Figure 3.5 (panels $\mathrm{j}-1$ ), the mutant protein stained with anti-HA antibody co-localizes in the nucleus as indicated by DAPI staining. Taken together, these results demonstrate that the mutant knock-in protein is expressed in the fetal liver and is localized to the nucleus. 

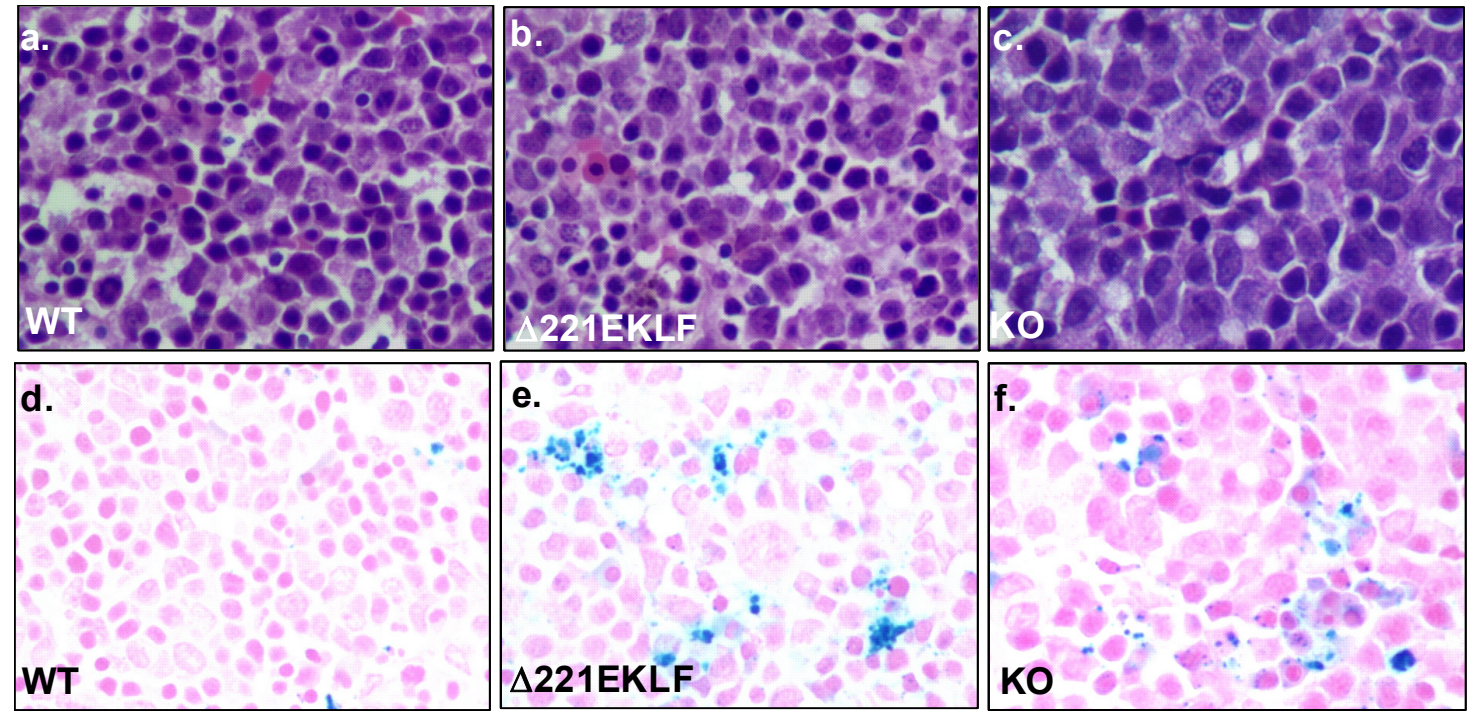

Figure 3.3. Accumulation of hemosiderin in fetal liver of mice expressing

$\triangle$ 221EKLF. Paraffin-embedded sections of fetal liver from E14.5 embryos: (a-c) hematoxylin and eosin stain and (d-f) Prussian blue stain. Hemosiderin is detected as blue deposits in the fetal liver. Original magnification x 400 . 
Figure 3.4. Expression of $\Delta 221 E K L F$ in fetal liver. (A) Top Panel: Schematic of the wild-type and knock-in alleles of EKLF. Arrows indicate the primers used in RT-PCR. Bottom Panel: Representative picture of agarose gel demonstrating that $\triangle 221 \mathrm{EKLF}$ transcripts are amplified only with RNA extracted from fetal liver of homozygous $\triangle 221$ EKLF embryos at E14.5. Additionally, downstream exons 2 and 3 of the endogenous EKLF locus are not transcribed in these animals. HPRT transcripts are detected as loading control. (B-C) Western blot analysis demonstrating that $\triangle 221 \mathrm{EKLF}$ knock-in protein is expressed in fetal liver. Whole cell extracts were prepared from fetal liver of E14.5 wild-type, $\Delta 221 \mathrm{EKLF}$ heterozygous, and $\Delta 221 \mathrm{EKLF}$ homozygous embryos. Whole cell extracts from J2e cell lines were included as controls. (B) An antiHA antibody was used to detect the HA-tagged mutant protein (HA- $\Delta 221 \mathrm{EKLF}$ ). (C) Anti-EKLF antibody generated in our laboratory (J.M.C. unpublished) was used to detect wild-type EKLF and mutant $\triangle 221$ EKLF proteins. $\beta$-actin expression was detected as loading controls. 
A.

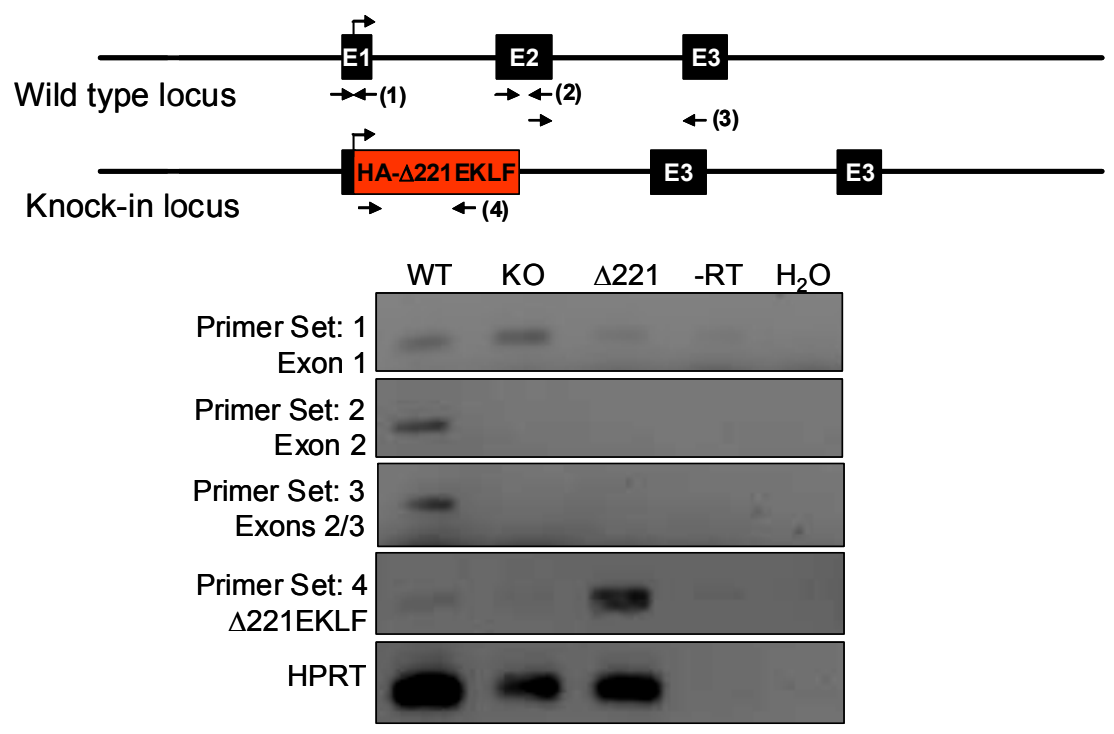

B.

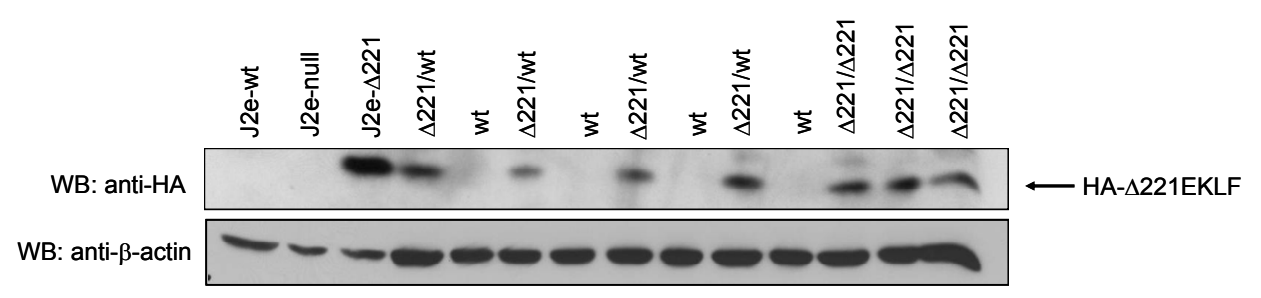

C.

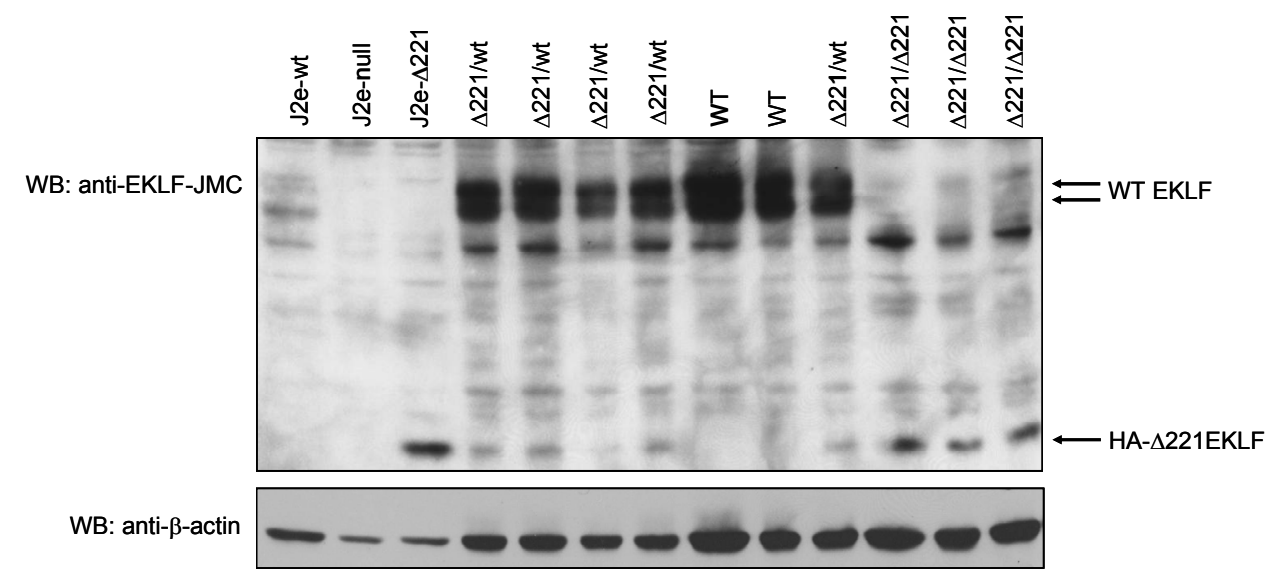




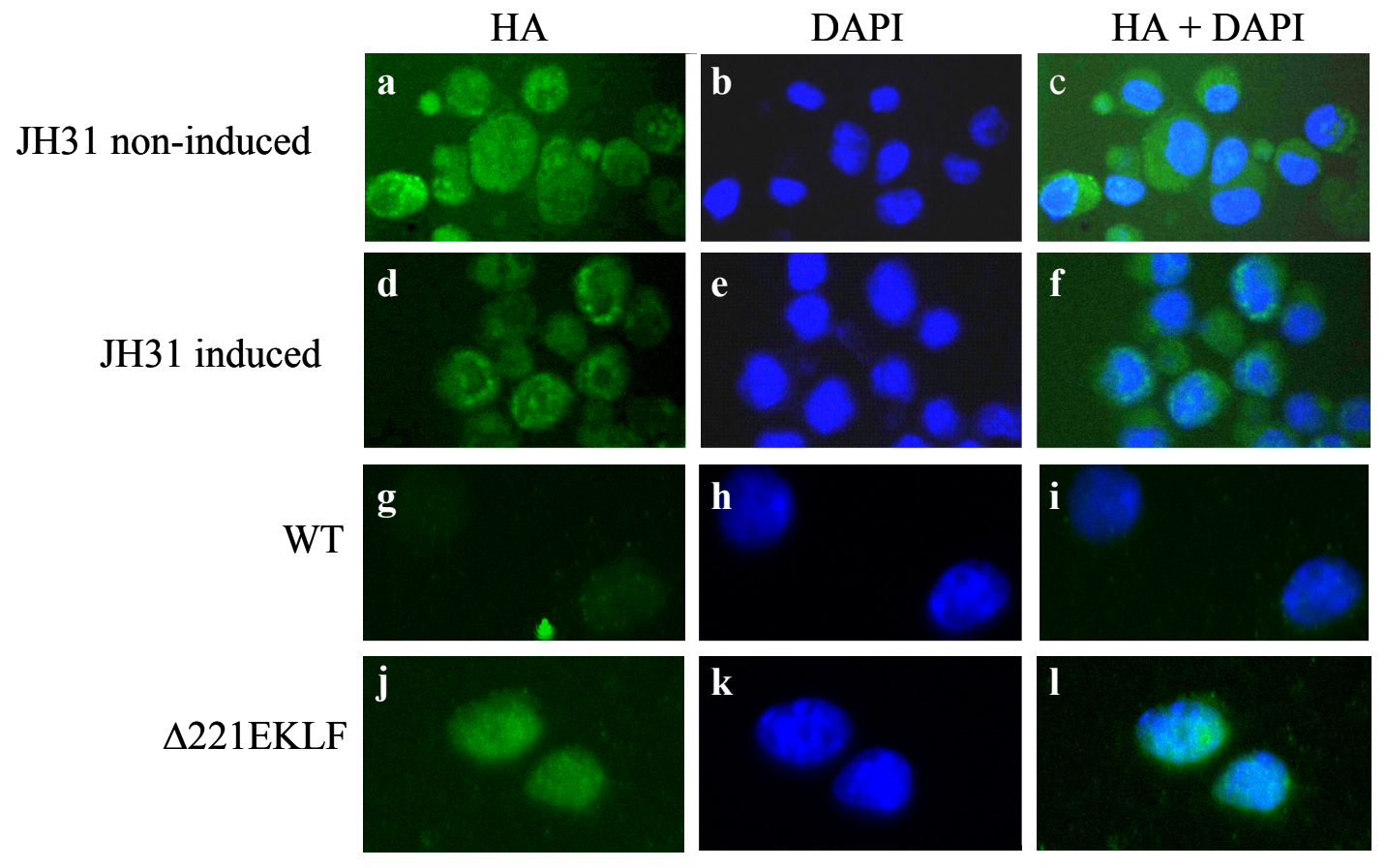

Figure 3.5. Localization of $\Delta 221 E K L F$ knock-in protein. Cytospin preparations of fetal liver erythroblasts from E14.5 $\triangle 221$ EKLF homozygous embryos were stained with anti-HA antibody $(\mathrm{j}-1)$. The slides were mounted with VECTASHIELD ${ }^{\circledR}$ mounting medium containing DAPI. Wild-type fetal liver erythroblasts, which lack the HA tag, served as a negative controls (g-i) while the inducible JH31 cellular system containing a HA-EKLF-ER fusion protein served as positive controls (a-f). In the absence of the ER ligand, HA-EKLF-ER protein is bound to chaperone proteins in the cytoplasm but can be translocated inside the nucleus in the presence of Tamoxifen. 


\section{Expression of $\triangle 212 \mathrm{EKLF}$ is sufficient for B-globin promoter remodeling}

To determine the functional consequences of $\triangle 221 \mathrm{EKLF}$ on the chromatin structure in vivo, nucleosomal remodeling at the $\beta$-globin promoter was determined by a real time PCR-based DNase I hypersensitivity assay (McArthur et al., 2001). Chromatin prepared from fetal liver nuclei was incubated with increasing amounts of the DNase I enzyme. DNA was purified and analyzed by real time PCR. The sensitivity is plotted as a percent of DNA remaining at different concentrations of DNase I and normalized to an internal control region from the neurofilament Nf-M gene located on murine chromosome 14. Nf-M is relatively resistant to DNase I digestion, although at higher concentrations of DNase I and longer incubation time period, the DNA at this region is digested eventually by DNase I. As shown in Figure 3.6, chromatin extracted from $\triangle 221 \mathrm{EKLF}$ fetal liver erythroblasts is sensitive to treatment with increasing amounts of DNase I at a level comparable to chromatin from derived from wild-type erythroblasts. In contrast, and consistent with previous observations (Wijgerde et al., 1996), chromatin derived from EKLF-null erythroblasts are relatively insensitive to DNase I treatment up to $18 \mathrm{U}$ of enzyme. The amount of sensitivity is erythroid cell-dependent, and the fetal liver has previously been determined to be greater than $20 \%$ hepatocytes at this stage (McArthur et al., 2001).

\section{Expression of $\triangle 221 \mathrm{EKLF}$ induces histone modifications at the $\beta$-globin promoter}

To further explore the role of $\triangle 221 \mathrm{EKLF}$ in reorganizing the chromatin architecture at the $\beta$-globin gene promoter, the acetylation and methylation patterns of histone $\mathrm{H} 3$ (H3) across the $\beta$-globin locus was determined by ChIP assays. Extending the results from the DNase I HS assay, I hypothesized that the $\triangle 221 \mathrm{EKLF}$ domain is sufficient to induce acetylation of histone $\mathrm{H} 3$ and methylation at K4. As shown in Figure 3.7 , histones were acetylated at wild-type levels at the $\beta$-globin promoter in $\Delta 221 \mathrm{EKLF}$ erythroblasts. Interestingly, the level of acetylation was comparable to EKLF-null erythroblasts at the 3 ' coding region of the $\beta$-globin gene in $\triangle 221 \mathrm{EKLF}$ erythroblasts. This observation is consistent with the idea of a lack of significant $\beta$-globin elongation in $\Delta 221 \mathrm{EKLF}$ erythroblasts (Sawado et al., 2003). By contrast, the acetylation pattern at the $\beta$-globin HS2 was comparable in wild-type, $\triangle 221 \mathrm{EKLF}$, and EKLF-null erythroblasts, suggesting acetylation of HS2 of the LCR occurs independently of EKLF. Similarly, analysis of the trimethylation pattern of lysine 4 of histone $\mathrm{H} 3$ revealed comparable levels of trimethylation at the $\beta$-globin HS2; however, trimethylation at the $\beta$-globin promoter was increased in $\triangle 221 \mathrm{EKLF}$ erythroblasts to a comparable level observed in wild-type erythroblasts (Figure 3.8). This modification, trimethylated H3K4, has been associated with active genes in vivo (Bernstein et al., 2005). Taken together, the data suggest that expression of the $\triangle 221 \mathrm{EKLF}$ domain is sufficient for full and appropriate chromatin remodeling activity in vivo. 


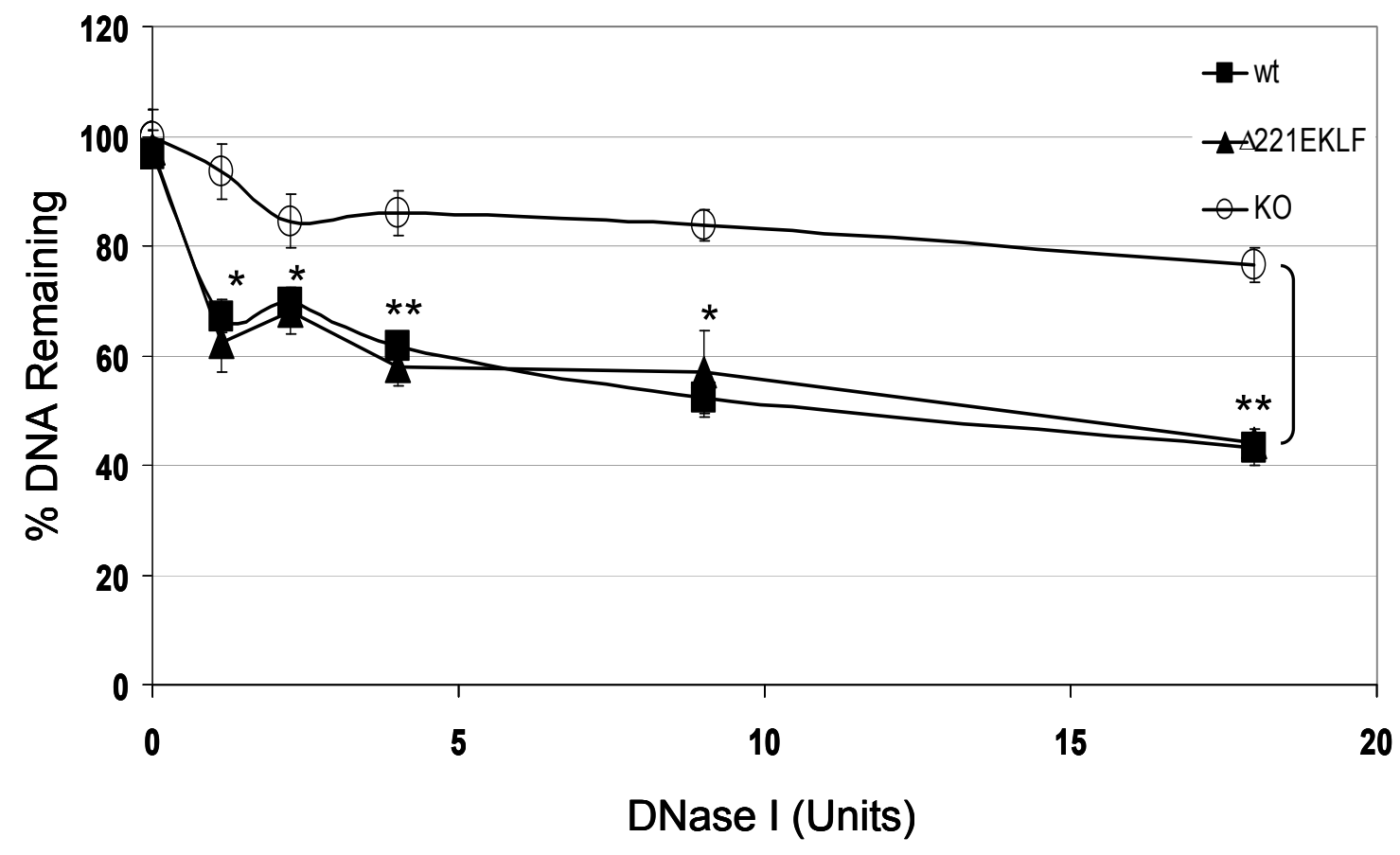

Figure 3.6. DNase I sensitivity at the $\beta$-globin promoter in $\triangle 221 E K L F$ fetal liver erythroblast. Plot of a quantitative real time PCR based DNase I hypersensitivity assay used to analyze the region of murine $\beta$ maj promoter in fetal liver from E14.5 wild-type, $\triangle 221 \mathrm{EKLF}$, and EKLF-null embryos. The results are plotted as the percent of DNA remaining after $5 \mathrm{~min}$ incubation with increasing amounts of DNase I enzyme normalized with the relatively insensitive Nf-M gene. Data shown are averages of at least three independent experiments. Error bars indicate the standard error of the mean. Asterisks denote $p$-value compared to KO. ${ }^{*}=p$-value $<0.05 ; * *=p$-value $<0.001$. 

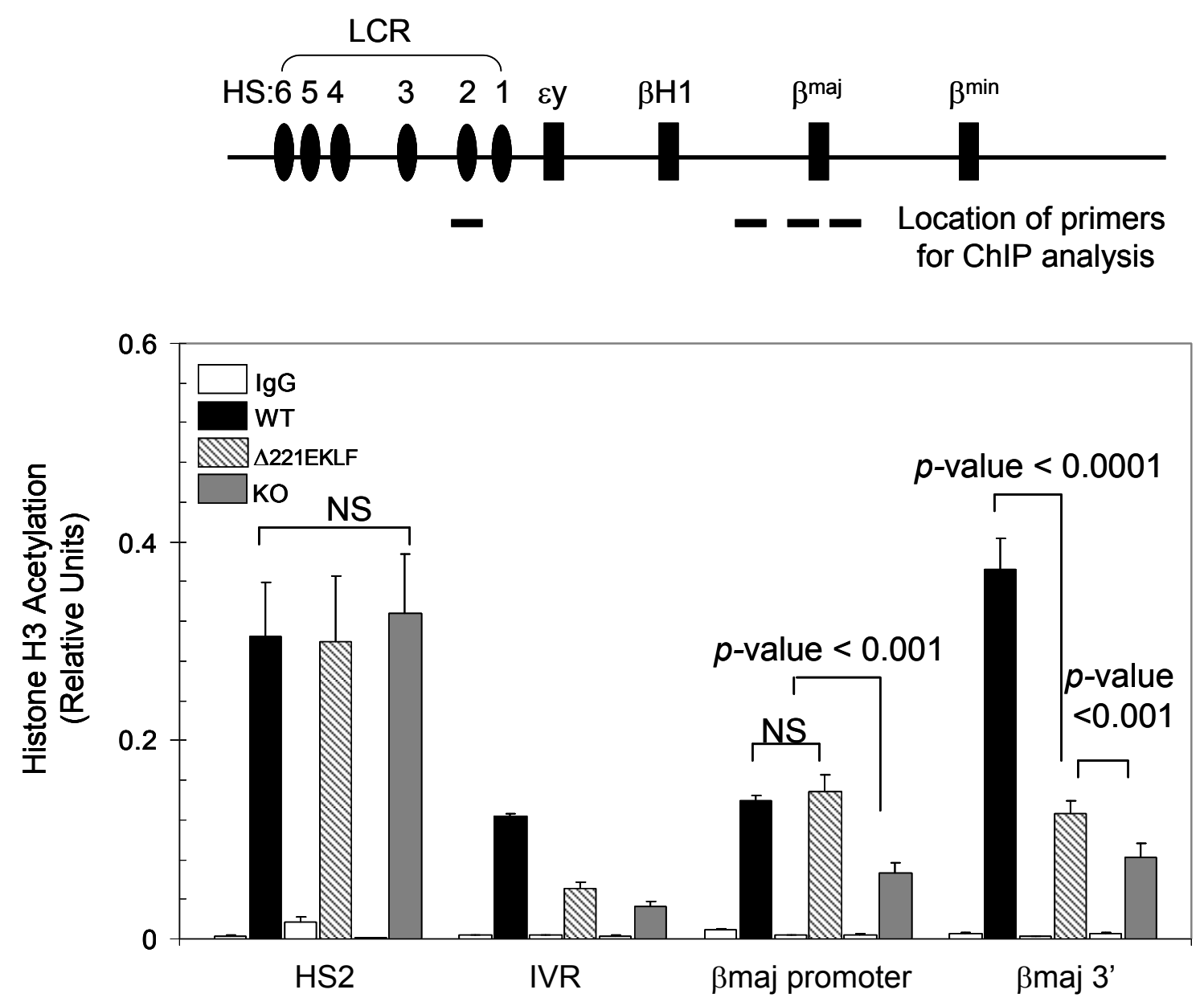

Figure 3.7. Global acetylation of histone $\mathbf{H} 3$ across the $\beta$-globin locus. Top panel: schematic of murine $\beta$-globin locus with location of primer pairs used in ChIP analysis denoted by solid lines. Bottom panel: pattern of histone $\mathrm{H} 3$ acetylation across the $\beta$ globin locus was analyzed by ChIP assay using anti-acetylated histone $\mathrm{H} 3(\mathrm{AcH} 3)$ antibody. Data shown are averages of at least five independent experiments performed in duplicate (mean $\pm \mathrm{SEM}$ ). $\mathrm{NS}=$ not significant. $\mathrm{IVR}=$ intervening region in the $\beta$-globin locus. 

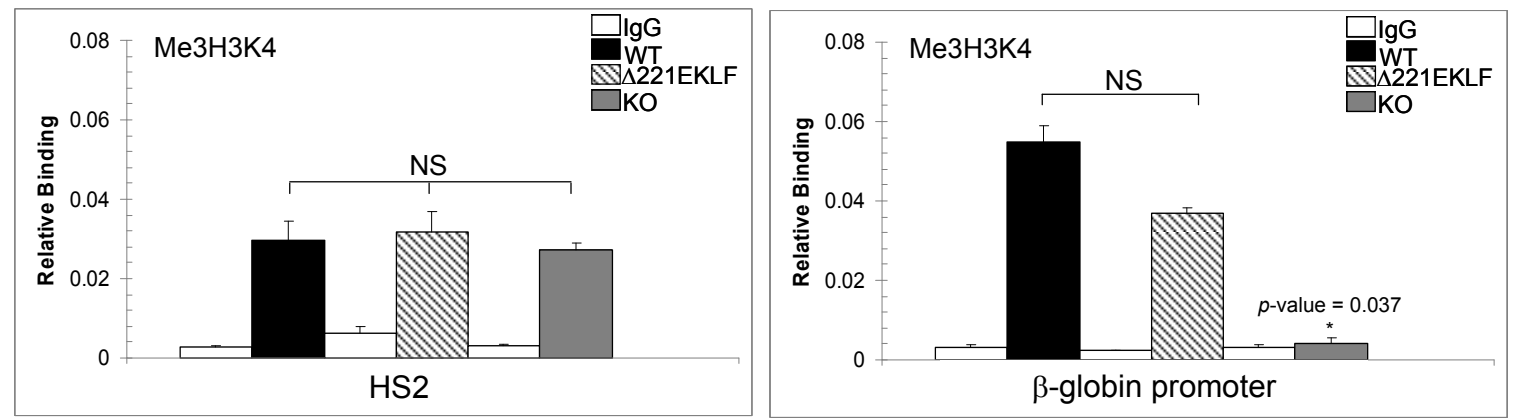

Figure 3.8. Trimethylation pattern at lysine 4 of histone H3. The trimethylation at lysine 4 of histone $\mathrm{H} 3$ (Me3H3K4) at the $\beta$-globin HS 2 and promoter sequences was analyzed by ChIP assay. Data shown are averages of at least five independent experiments performed in duplicate (mean $\pm \mathrm{SEM}$ ). NS = not significant. 


\section{$\triangle 221 E K L F$ binds to erythroid-specific gene loci}

The zinc finger DNA binding domain of EKLF recognizes and binds to the consensus sequence $\mathrm{CCNCNCCCN}$ found at many erythroid gene loci. To explore whether the mutant $\triangle 221$ EKLF protein was capable of binding to known EKLF regulatory sequences in vivo, chromatin immunoprecipitation (ChIP) was performed with a HA-specific anti-serum. In contrast to erythroid cells lacking EKLF, fetal liver erythroblasts expressing the $\triangle 221 \mathrm{EKLF}$ protein demonstrated appropriate binding of the chromatin remodeling encoding domain to known-EKLF regulatory sequences at the $\beta$ globin locus, but not to non-erythroid gene promoters (i.e. RPII215). As shown in Figure $3.9, \Delta 221 \mathrm{EKLF}$ protein binds to the $\beta$-globin HS2, the $\beta$-globin gene promoter as well as to the promoters of two erythroid genes, AHSP (Pilon et al., 2006) and $\alpha$-globin (Shyu et al., 2005). In contrast, the mutant protein did not bind to regions that do not contain EKLF binding sites, including an intervening region in the $\beta$-globin locus (IVR), downstream region of the $\beta$-globin gene, and the non-erythroid gene RPII215 (Im et al., 2005). The RPII215 gene, located on murine chromosome 11, encodes the largest subunit of RNA polymerase II (Pravtcheva et al., 1986). These results confirm that the mutant protein expressed in fetal liver is capable of binding to the EKLF regulatory sequences in vivo.

\section{Expression of $\triangle 221 \mathrm{EKLF}$ is insufficient for high-level $\beta$-globin transcription}

EKLF is essential for $\beta$-globin gene transcription. Although previous cellular studies have demonstrated that a larger domain of EKLF is required and sufficient to activate $\beta$-globin gene transcription (Brown et al., 2002), it is not clear what determinants are required in vivo. To determine if expression of $\Delta 221 \mathrm{EKLF}$ was sufficient for activation of gene transcription in vivo, the expression level of the adult $\beta$-globin gene in $\triangle 221 \mathrm{EKLF}$ fetal liver erythroblasts from E14.5 embryos were analyzed by real time RTPCR. Similar to complete loss of EKLF, there was minimal gene expression, or transcription as assayed by primary transcript analysis, of the $\beta$-globin gene in $\triangle 221 \mathrm{EKLF}$ erythroblasts compared to wild-type erythroblasts (Figure 3.10). These results suggest that additional amino terminal sequences are required for EKLFdependent gene activation in vivo. Moreover, the lack of $\beta$-transcripts suggests that these animals succumbed in utero, at least in part, from a severe anemia in a similar manner to EKLF-null animals (Nuez et al., 1995; Perkins et al., 1995).

\section{Differential binding of factors at the $\beta$-globin LCR and promoter}

The failure of $\triangle 221 \mathrm{EKLF}$ to activate $\beta$-globin transcription, despite nearly normal promoter remodeling as determined by DNase I HS formation and hyperacetylation of histones at the $\beta$-globin promoter, led me to explore the role of $\triangle 221 \mathrm{EKLF}$ in factor recruitment to the $\beta$-globin LCR and promoter. First, occupancy of PolII at the $\beta$-globin locus was examined by ChIP assay with anti-PolII antibody capable of detecting both phosphorylated and unphosphorylated forms of PolII. Phosphorylation of PolII serine 5 
A.

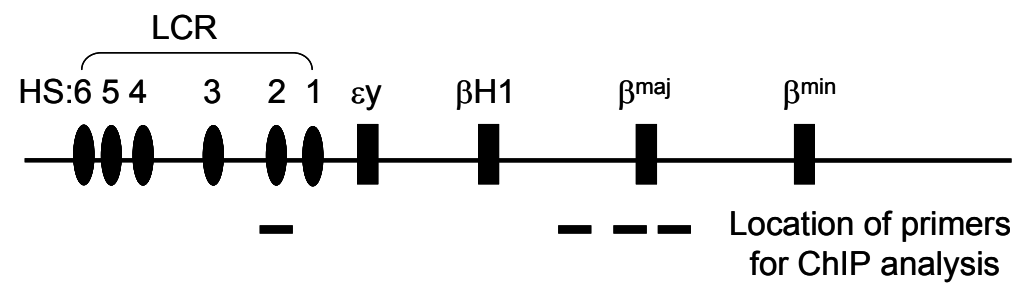

B.

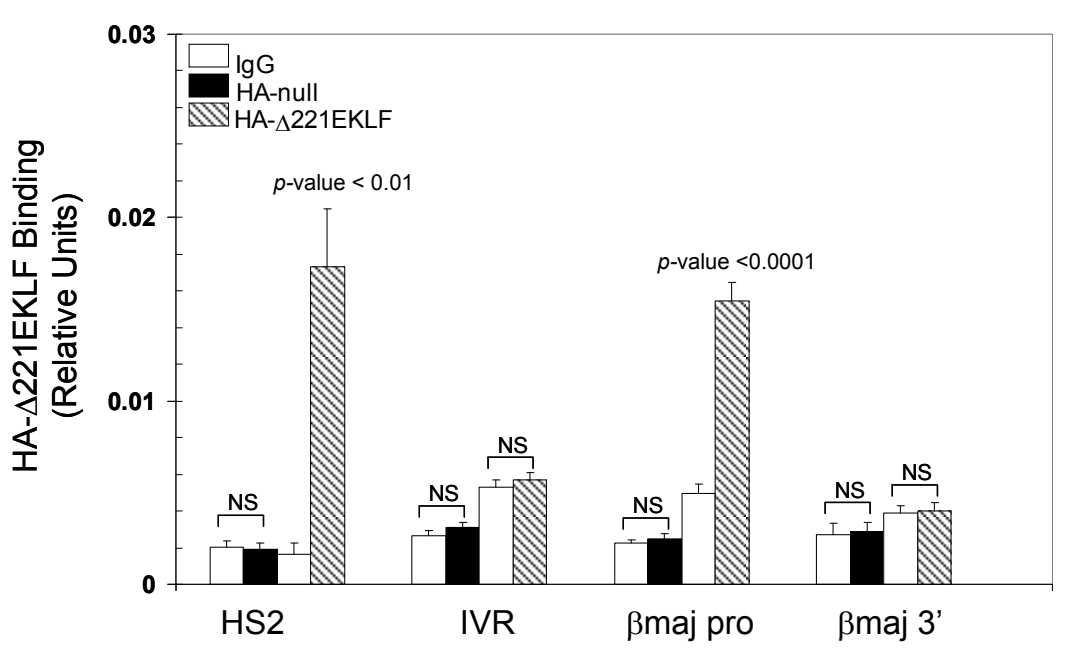

C.

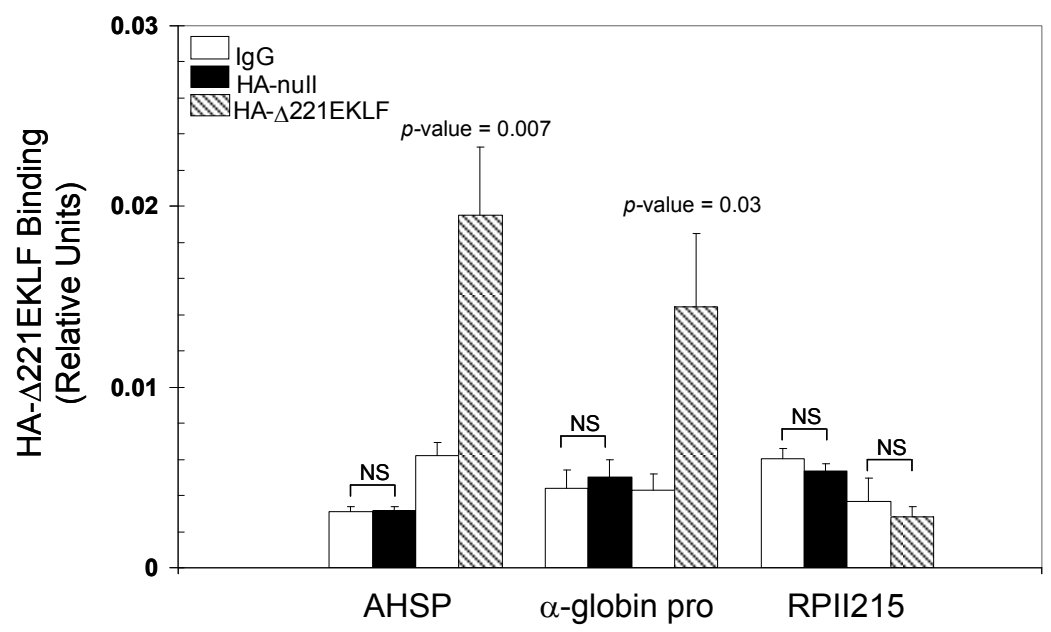

Figure 3.9. Differential binding of $\triangle 221 E K L F$ to erythroid-specific gene loci. (A) Schematic of murine $\beta$-globin locus with location of primer pairs used in ChIP analysis denoted by solid lines. (B) The binding of $\triangle 221 \mathrm{EKLF}$ across the $\beta$-globin locus was examined using ChIP assays with anti-HA specific anti-serum. (C) The binding of $\triangle 221 \mathrm{EKLF}$ at other erythroid-specific gene promoters (positive controls) and a nonerythroid gene promoter (negative control). Data shown are averages of at least five independent experiments performed in duplicate (mean $\pm \mathrm{SEM}$ ). NS $=$ not significant. 

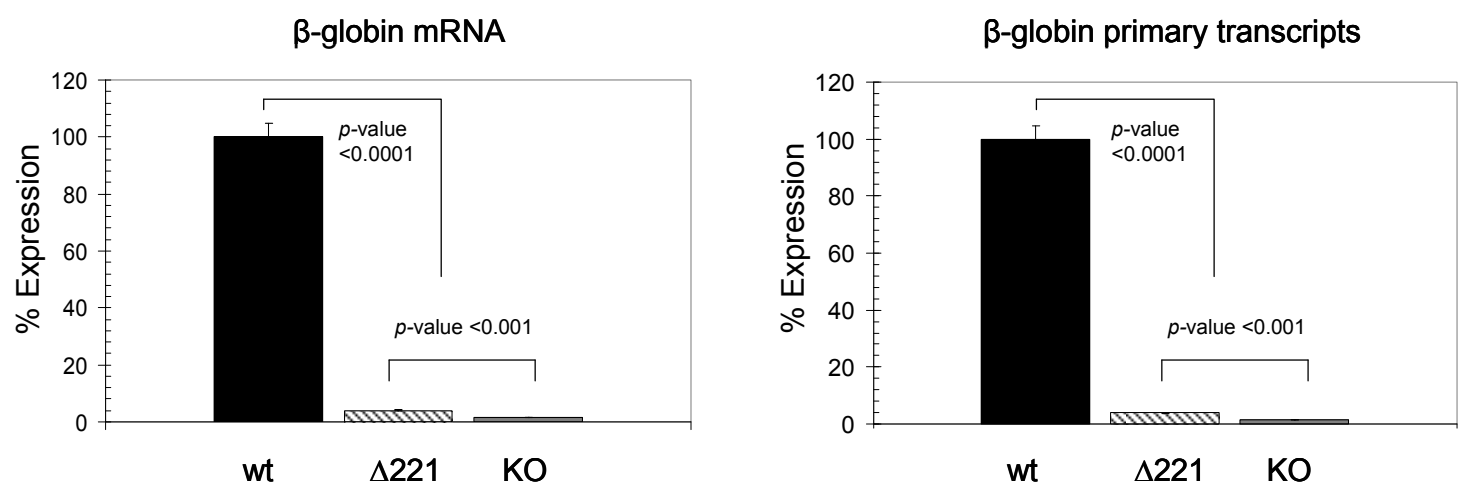

Figure 3.10. Lack of $\beta$-globin transcripts in $\triangle 221 \mathrm{EKLF}$ fetal liver erythroblasts in vivo. RNA was extracted from fetal liver of E14.5 wild-type, $\triangle 221 \mathrm{EKLF}$, and EKLFnull embryos and treated with Turbo DNase prior to cDNA synthesis. $\beta$-globin gene expression (as determined by mRNA levels) and transcription (as assayed by primary transcripts) were analyzed by real time RT-PCR. Data shown are averages of at least five independent experiments (mean \pm SEM). 
is an essential step in transition from a stalled to an elongating polymerase (Phatnami \&Greenleaf, 2006). Not surprisingly, there was increased occupancy of PolII at the $\beta$ globin promoter and 3' of the gene in wild-type erythroblasts (Figure 3.11), consistent with active $\beta$-globin transcription. In contrast, minimal RNA polymerase II occupancy was observed at the $\beta$-globin promoter in $\triangle 221 \mathrm{EKLF}$ expressing cells of a similar chronology. Furthermore, the amount of PolII at the 3' region of the $\beta$-globin gene was not significantly increased, consistent with the idea of a paused PolII complex at the $\beta$ globin promoter (Phatnami \& Greenleaf, 2006). This observation is supported by the $\beta$ globin transcript data, demonstrating a significant reduction in $\beta$-globin transcripts in $\triangle 221 \mathrm{EKLF}$ erythroblasts as compared with wild-type erythroblasts (Figure 3.10). When compared to EKLF-null cells, occupancy of PolII at the $\beta$-globin promoter was approximately 3 -fold higher in $\triangle 221 \mathrm{EKLF}$ cells ( $\mathrm{p}$-value $<0.0001$ ). By contrast, similar levels of PolII were recruited to HS2 of the LCR in wild-type, $\triangle 221 \mathrm{EKLF}$, and EKLFnull cells, consistent with the idea that EKLF does not mediate this step. Furthermore, this result is not surprising given previous studies have demonstrated that recruitment of PolII to the LCR occurs independent of recruitment to and activation of the $\beta$-globin promoter (Johnson et al., 2003). Importantly, my results support a role for EKLF in recruitment of PolII to the $\beta$-globin promoter, as has been previously described for GATA-1 and NF-E2 (Johnson et al., 2001).

The $\beta$-globin promoter contains numerous binding sites for erythroid-specific and ubiquitous transcription factors that regulate appropriate high-level expression of the $\beta$ globin gene. EKLF, GATA-1, and NF-E2 are among the best characterized transcriptional activators and have been shown to bind the $\beta$-globin promoter sequences and DNase HS sites (HS) at the LCR (Mahajan et al., 2007). To elucidate the role of $\triangle 221 \mathrm{EKLF}$ in EKLF-mediated transactivation of the $\beta$-globin gene, I analyzed the recruitment GATA- 1 and NF-E2 to the HS2 of the LCR and $\beta$-globin promoter in $\triangle 221 E K L F$ expressing fetal liver erythroblasts (Figure 3.12). The occupancy of NF-E2 and GATA- 1 at an intervening region (IVR) on the $\beta$-globin locus and the downstream region of the $\beta$-globin gene were included as negative controls. Chromatin immunoprecipitation analysis revealed a comparable level of NF-E2 occupancy at the $\beta$ globin HS2; however, NF-E2 occupancy at the $\beta$-globin promoter was significantly reduced in $\triangle 221 \mathrm{EKLF}$ erythroblasts as compared with wild-type erythroblasts (Figure $3.12 \mathrm{~A}$ ). These results are consistent with previous reports that demonstrate NF-E2 recruitment to HS2 of the LCR and $\beta$-globin promoter is essential for activation of $\beta$ globin transcription (Forsberg et al., 2000; Sawado et al., 2003). Similarly, GATA-1 occupancy at the $\beta$-globin HS2 was comparable in wild-type, $\triangle 221 \mathrm{EKLF}$, and EKLF-null erythroblasts (Figure 3.12B). In contrast, GATA-1 occupancy at the $\beta$-globin promoter was significantly reduced in $\triangle 221 \mathrm{EKLF}$ as compared to wild-type erythroblasts. These observations are consistent with the role for GATA-1 at HS2 of the LCR in co-activator and PolII recruitment to the $\beta$-globin promoter (Cho et al., 2008). Additionally, my results in $\triangle 221 \mathrm{EKLF}$-expressing cells suggest that a larger amino terminal domain of EKLF is required and necessary for recruitment of GATA-1 and NF-E2 to the $\beta$-globin promoter. 


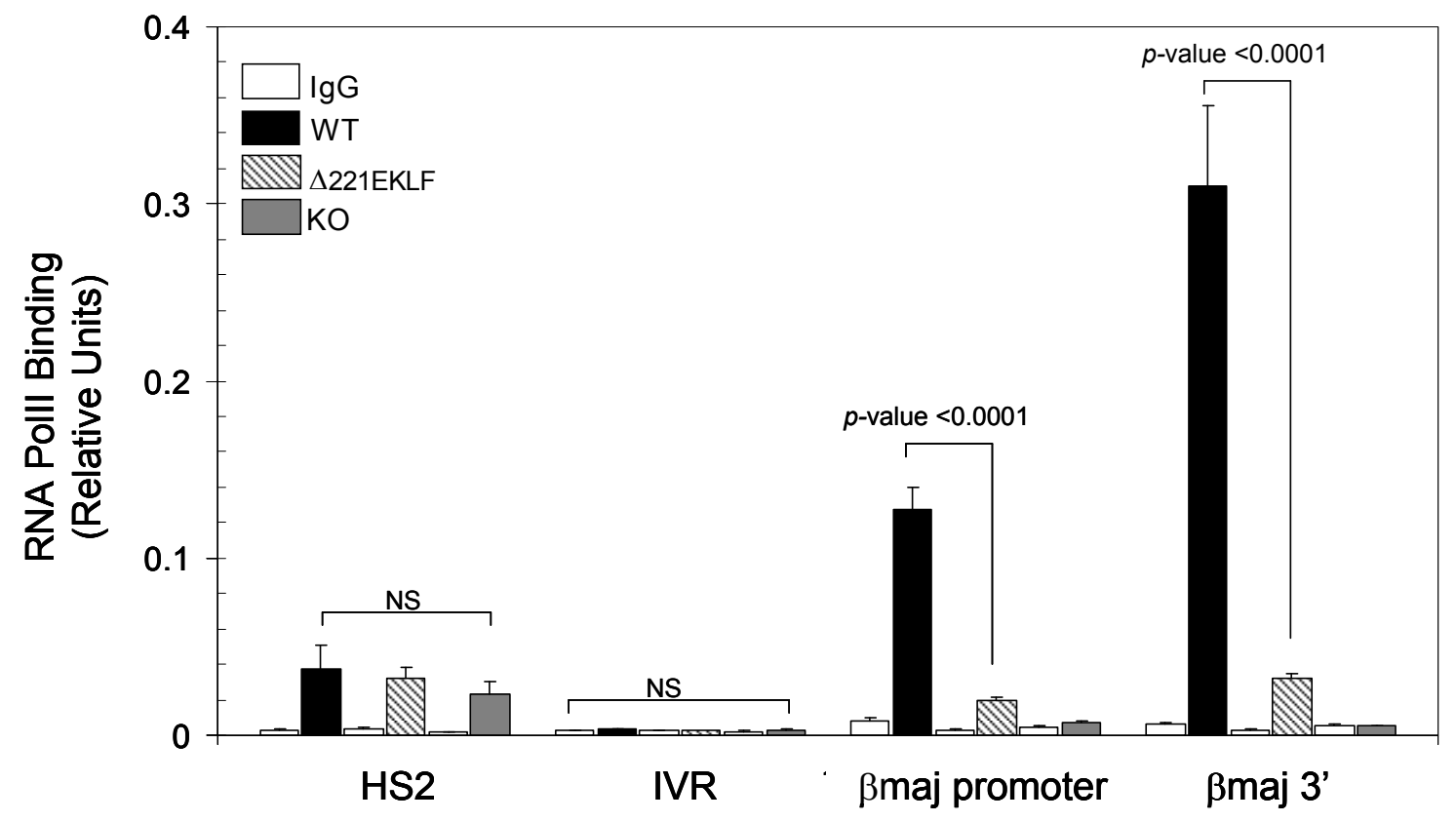

Figure 3.11. Lack of RNA polymerase at the $\beta$-globin promoter in $\triangle 221 E K L F$ expressing cells. The amount of total PolII occupancy across the $\beta$-globin locus was examined by ChIP analysis with the polyclonal anti-PolII antibody. Data shown are averages of at least five independent experiments performed in duplicate (mean \pm SEM). $\mathrm{NS}=$ not significant. 
A.

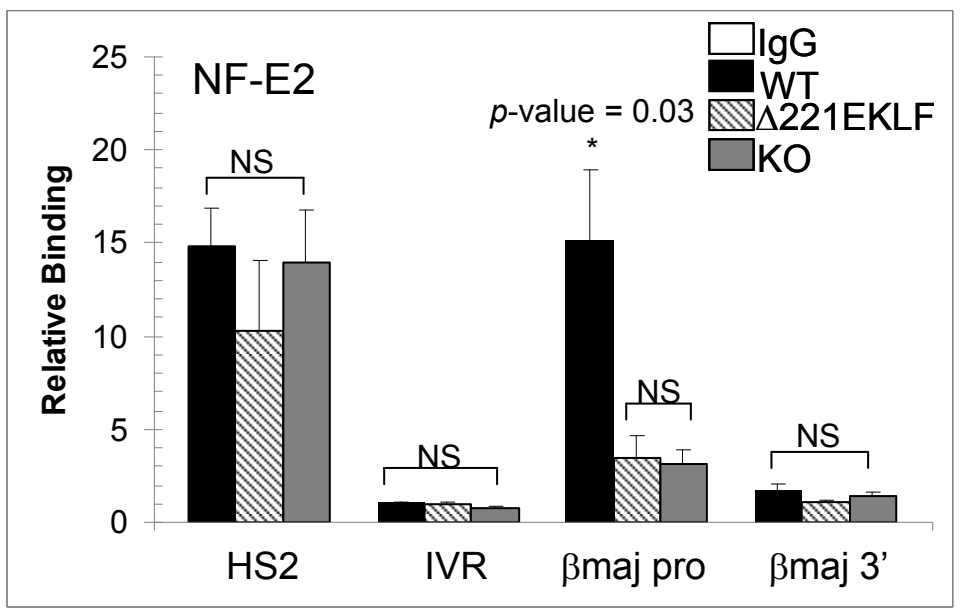

B.

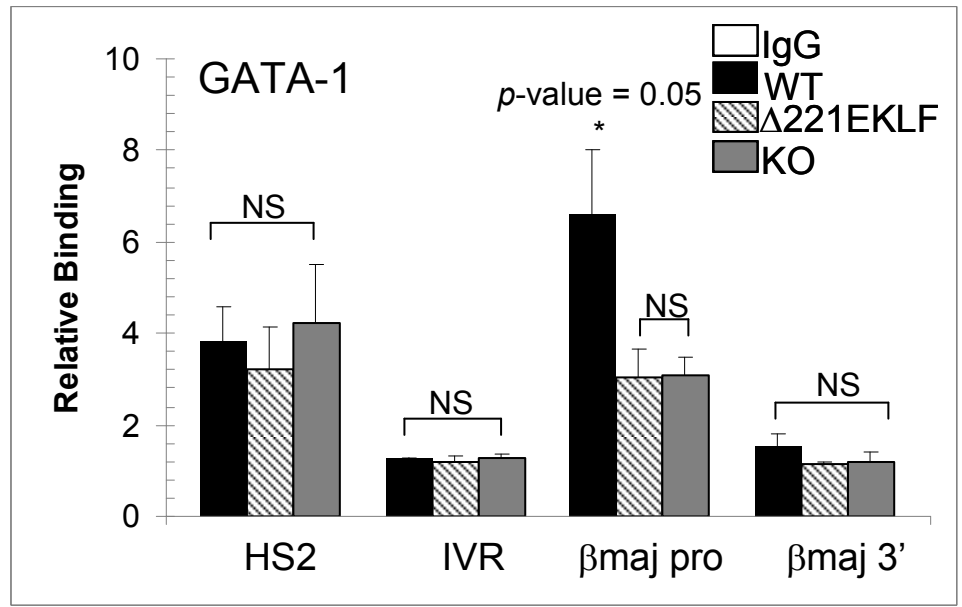

Figure 3.12. Binding of erythroid-specific transcription factors at the $\beta$-globin promoter in vivo. Binding of (A) NF-E2 and (B) GATA- 1 across the $\beta$-globin locus was analyzed by ChIP assay in fetal liver from E14.5 wild-type, $\triangle 221 \mathrm{EKLF}$, and EKLF-null embryos. Data shown are averages of at least three independent experiments performed in duplicate (mean $\pm \mathrm{SEM})$. NS $=$ not significant. 


\section{DISCUSSION}

The studies in this chapter are aimed at exploring the hypothesis that expression of $\triangle 221 \mathrm{EKLF}$ alone is sufficient for altering the local chromatin structure at the $\beta$-globin promoter in vivo. Furthermore, these studies have provided significant insights into EKLF-mediated activation of $\beta$-globin transcription in vivo.

\section{Expression of $\triangle 221 \mathrm{EKLF}$ is not sufficient to rescue the EKLF-null phenotype}

I have demonstrated in the present study that expression of $\triangle 221 \mathrm{EKLF}$ in vivo cannot rescue the EKLF null phenotype. Like mice lacking EKLF (Nuez et al., 1995; Perkins et al., 1995), 2221 EKLF mice exhibit a phenotype consistent with ineffective erythropoiesis and die by E15.5 of gestation. The $\triangle 221 \mathrm{EKLF}$ embryo is anemic with few hemoglobin-containing erythroblasts in the fetal liver, consistent with a block in erythroid differentiation. Expression of $\triangle 221 \mathrm{EKLF}$ protein has been confirmed in vivo. Although the mutant $\triangle 221 E K L F$ protein appears to be expressed at lower levels than wild-type EKLF protein, this alone cannot account for the observed phenotype of $\triangle 221 \mathrm{EKLF}$ mice given that the mutant protein retains full and appropriate chromatin remodeling properties (see discussion below). Previous studies in our laboratory have shown that this domain of EKLF cannot activate $\beta$-globin gene transcription in erythroid cell lines (Brown et al., 2002). Thus, it was anticipated that expression of $\triangle 221 \mathrm{EKLF}$ in a whole animal model would result in embryonic lethality reminiscent of the EKLF-null mice.

\section{Expression of $\triangle 221 \mathrm{EKLF}$ is sufficient for nucleosomal remodeling and histone modifications in vivo}

Previous studies analyzing the distinct domains of EKLF in erythroid cell lines demonstrated that chromatin remodeling and transcriptional activation functions of EKLF are separable (Brown et al., 2002). Conclusions from these studies are that sequences between amino acid 221-253 are necessary and sufficient for chromatin remodeling at the $\beta$-globin promoter while additional $\mathrm{N}$-terminal amino acid sequences are required for transactivation of $\beta$-globin gene transcription. Moreover, expression of $\triangle 221 \mathrm{EKLF}$ in an erythroid cell line results in DNase I HS formation at the $\beta$-globin promoter to wild-type levels.

My studies expand on these conclusions and provide novel results on the chromatin remodeling property of EKLF in vivo. Indeed, DNase I hypersensitivity assays reveal that chromatin derived from $\triangle 221 \mathrm{EKLF}$ fetal liver erythroblasts is as sensitive to DNase I treatment as wild-type chromatin, whereas chromatin from EKLF-null erythroblasts is relatively insensitive (Figure 3.6). Because the degree of DNase I sensitivity corresponds with nucleosomal remodeling (Steger \& Workman, 1996; Pazin et al., 1997), the data strongly suggest that expression of $\triangle 221 \mathrm{EKLF}$ is sufficient for nucleosomal remodeling at the $\beta$-globin promoter in vivo. Previous studies analyzing the role of EKLF in chromatin remodeling have relied on in vitro chromatin reconstitution 
assays. These studies have identified a SWI/SNF-containing multiprotein complex, E$\mathrm{RC} 1$, which interacts with the zinc finger DNA binding domain of EKLF (Armstrong et al., 1998; Kadam et al., 2000). This interaction results in chromatin remodeling and transcriptional activation of a chromatinized $\beta$-globin template. Additional evidence supporting this interaction comes from studies demonstrating that EKLF interacts with BRG1, a subunit of E-RC1 (Zhang et al., 2001). However, evidence for a direct interaction between EKLF and SWI/SNF components in vivo is still lacking; however, BRG1 is a highly attractive candidate to explore.

Similarly, I show that expression of $\triangle 221 \mathrm{EKLF}$ is sufficient to induce histone modifications in vivo. First, global acetylation of histone $\mathrm{H} 3(\mathrm{AcH} 3)$ at the $\beta$-globin promoter is increased in $\triangle 221 \mathrm{EKLF}$ erythroblasts to an equivalent level as observed in wild-type erythroblasts (Figure 3.7). Similarly, trimethylation at lysine 4 of histone $\mathrm{H} 3$ at the $\beta$-globin promoter is increased to wild-type levels in $\triangle 221 \mathrm{EKLF}$ erythroblasts (Figure 3.8). It is known that higher acetylation particularly of histones $\mathrm{H} 3$ and $\mathrm{H} 4$ are associated with the 'opening' of chromatin for transcription (Yan \& Boyd, 2006). Furthermore, trimethylation at lysine 4 of histone $\mathrm{H} 3$ has been associated with promoters of active genes (Bernstein et al., 2005). My results not only extend these observations to include EKLF, but also provide evidence that the $\triangle 221$ EKLF domain alone is sufficient for highlevel $\mathrm{H} 3$ acetylation at the $\beta$-globin promoter. EKLF has previously been shown to interact with the histone acetyltransferase, CREB binding protein (CBP) (Zhang \& Bieker, 1998; Zhang et al., 2001), which in addition to acetylation of EKLF protein can also increase acetylation of histones (Blobel, 2002). Thus, it is highly tempting to speculate that $\triangle 221 \mathrm{EKLF}$ induces $\mathrm{CBP}$ recruitment and increases the acetylation of histone $\mathrm{H} 3$ at the $\beta$-globin promoter in the absence of $\beta$-globin transcription.

NF-E2 and GATA-1 have been implicated in mediating $\mathrm{H} 3$ acetylation and methylation at the $\beta$-globin promoter (Kiekhaefer et al., 2002; Kiekhaefer et al., 2004; Demers et al., 2007). Using cell lines, these studies have demonstrated that GATA-1 induces $\mathrm{H} 3$ acetylation (Kiekhaefer et al., 2002) while NF-E2 induces H3K4 trimethylation at the $\beta$-globin promoter (Kiekhaefer et al., 2004; Demers et al., 2007). In contrasts, my study in primary erythroblasts suggests EKLF, and more importantly the $\triangle 221 \mathrm{EKLF}$ domain, is required and sufficient for $\mathrm{H} 3$ acetylation and $\mathrm{H} 3 \mathrm{~K} 4$ trimethylation at the $\beta$-globin promoter in vivo. As shown in Figure 3.12, there is modest NF-E2 and GATA-1 occupancy at the $\beta$-globin promoter in $\triangle 221 \mathrm{EKLF}$ and EKLF-null cells when compared to wild-type erythroblasts. However, in contrast to complete loss of EKLF, expression of $\triangle 221 \mathrm{EKLF}$ results in $\mathrm{H} 3$ acetylation and $\mathrm{H} 3 \mathrm{~K} 4$ trimethylation to a level comparable to wild-type in $\triangle 221 \mathrm{EKLF}$ erythroblasts. While it is not possible to discount entirely the role of GATA- 1 and NF-E2 in histone modifications at the $\beta$-globin promoter, my results are consistent with the idea that $\triangle 221 \mathrm{EKLF}$ is a critical mediator of this process. It is possible the basal levels of GATA-1 and NF-E2 at the $\beta$-globin promoter require $\triangle 221 \mathrm{EKLF}$ to induce $\mathrm{H} 3$ acetylation and $\mathrm{H} 3 \mathrm{~K} 4$ trimethylation in vivo. 


\section{Nucleosomal remodeling and histone modification are not sufficient for recruitment of erythroid-specific transcription factors to the $\beta$-globin promoter and activated $\beta$ - globin transcription}

In the present studies, I show that the level of $\beta$-globin transcripts in the $\triangle 221 \mathrm{EKLF}$ mice is less than $5 \%$ of wild-type mice (Figure 3.10). These results are consistent with our previous analysis of $\triangle 221 \mathrm{EKLF}$ expressed in an erythroid cell line (Brown et al., 2002). Furthermore, the low levels of $\beta$-globin gene transcription despite high levels of $\mathrm{H} 3$ acetylation and nucleosomal remodeling at the $\beta$-globin promoter is highly reminiscent of previous studies in mice homozygous for a targeted deletion of the LCR (Bender et al., 2000). These studies have demonstrated that deletion of the LCR resulted in a severe reduction of $\beta$-globin transcription with concomitant decreased PolII occupancy at the $\beta$-globin promoter (Bender et al., 2000; Sawado et al., 2003). However, the $\beta$-globin promoter remains hyperacetylated and nuclease sensitive in LCR deletion and wild-type mice. Taken together, these observations suggest that nucleosomal remodeling and modification of histones are not sufficient to achieve high level $\beta$-globin transcription in vivo, consistent with lack of LCR recruitment to the promoter. Furthermore, the reduced level of PolII occupancy in $\triangle 221 E K L F$ mice is consistent with the idea of a long range transfer of PolII from the LCR to the promoter to stimulate transcription as has been previously described by Johnson et al. (2001).

GATA-1 and NF-E2 have both been implicated in $\beta$-globin gene activation (Kim $\&$ Bresnick, 2007). The present studies demonstrate that EKLF is essential for recruitment of these factors to the $\beta$-globin promoter but not HS2 of the LCR. Using ChIP analysis, both GATA-1 and NF-E2 are recruited normally to HS2 of the LCR in $\triangle 221 \mathrm{EKLF}$ and EKLF-null erythroblasts. However, there is significantly lower GATA-1 and NF-E2 occupancy at the $\beta$-globin promoter in $\triangle 221 \mathrm{EKLF}$ and EKLF-null erythroblasts as compared to wild-type erythroblasts. My results are novel and unique to $\triangle 221 \mathrm{EKLF}$-expressing cells, providing strong support for an essential role for EKLF in recruitment of GATA- 1 and NF-E2 to the $\beta$-globin promoter. These results are contrasted with previous studies that have demonstrated GATA-1 and NF-E2 occupy the $\beta$-globin promoter in erythroid cells from mice lacking the LCR (Sawado et al., 2003; Vakoc et al., 2005), suggesting that recruitment of GATA-1 and NF-E2 to the $\beta$-globin promoter occur independent of the LCR. The results in the present study are consistent with two possible mechanisms of transcriptional factor recruitment to the $\beta$-globin promoter. The first mechanism proposes that recruitment of GATA-1 and NF-E2 to the $\beta$-globin promoter is EKLF-dependent, requiring a larger amino terminal domain of EKLF. An alternative mechanism is that GATA-1 and NF-E2 are first recruited to the $\beta$ globin LCR (EKLF-independent) and then transferred to the $\beta$-globin promoter (EKLFdependent). Studies to examine LCR $/ \beta$-globin promoter interaction would provide additional insights into the mechanism of transcriptional factor recruitment to the $\beta$ globin promoter to stimulate gene transcription. 


\section{A model for $\triangle 221 E K L F$ in $\beta$-globin promoter remodeling}

Although in vitro and cellular studies have analyzed the coordinated recruitment of transcription factors and coactivators required for high level $\beta$-globin transcription, much knowledge remains to be gained from studying EKLF-mediated chromatin remodeling and $\beta$-globin transcription in vivo. The results from the present studies in $\triangle 221 E K L F$ mice indicate that chromatin remodeling and histone modifications are not sufficient to achieve high-level $\beta$-globin transcription in vivo. Furthermore, these studies suggest that $\triangle 221 \mathrm{EKLF}$ is sufficient for $\beta$-globin promoter remodeling but additional amino terminal sequences are necessary for recruitment of transcriptional factors to the promoter. A proposed model for the role of $\triangle 221 \mathrm{EKLF}$ in promoter remodeling is shown in Figure 3.13. First, $\mathrm{H} 3$ acetylation and $\mathrm{H} 3 \mathrm{~K} 4$ trimethylation at HS2 of the $\beta$-globin LCR occurs independent of EKLF. Furthermore, GATA-1, NF-E2, and PolII are recruited normally in the absence of EKLF. In contrast, these events at the $\beta$-globin promoter are EKLF-dependent. Similar to the observation in wild-type erythroblasts, expression of $\triangle 221 \mathrm{EKLF}$ results in an open chromatin configuration with histone modifications consistent with an active gene promoter. However, recruitment of GATA1 , NF-E2, and PolII to stimulate transcription at the $\beta$-globin promoter requires additional amino terminal sequences of EKLF not included in the $\triangle 221 \mathrm{EKLF}$ domain. In contrast, the $\beta$-globin promoter in EKLF-null erythroblasts remains in a closed chromatin configuration consistent with a repressed $\beta$-globin promoter. It is unclear at this juncture what role $\Delta 221 \mathrm{EKLF}$ plays in LCR/ $\beta$-globin promoter interaction. Thus, further dissection of the role of $\triangle 221 \mathrm{EKLF}$ in modulation of the LCR/ $\beta$-globin promoter interaction and recruitment of other factors at the $\beta$-globin promoter will provide additional fundamental insights into EKLF-mediated chromatin remodeling and $\beta$-globin transcription in vivo. 
Figure 3.13. Models of $\boldsymbol{\beta}$-globin promoter remodeling in erythroid cells. Histone modifications and recruitment of GATA-1, NF-E2, and PolII to the $\beta$-globin LCR does not require EKLF. In contrast, the events at the $\beta$-globin promoter are EKLF-dependent. (A) In wild-type erythroblasts, EKLF induces $\mathrm{H} 3$ hyperacetylation and $\mathrm{H} 3 \mathrm{~K} 4$ trimethylation. Transcriptional factors (GATA-1, NF-E2, PolII) are recruited to the $\beta$ globin promoter to stimulate $\beta$-globin gene transcription. (B) In $\Delta 221 \mathrm{EKLF}$-expressing erythroblasts, $\triangle 221 \mathrm{EKLF}$ alone is sufficient to induce $\mathrm{H} 3$ hyeracetylation and H3K4 trimethylation. However, additional amino terminal sequences of EKLF are required for recruitment of GATA-1, NF-E2, and PolII to stimulate $\beta$-globin gene transcription. (C) In the absence of EKLF, the $\beta$-globin promoter remains in a closed chromatin configuration consistent with a repressed gene promoter. 
A. Wild-type

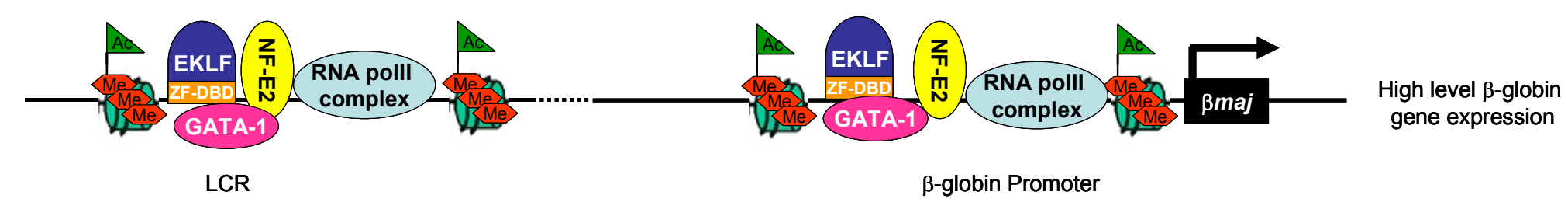

B. $\triangle 221 \mathrm{EKLF}$
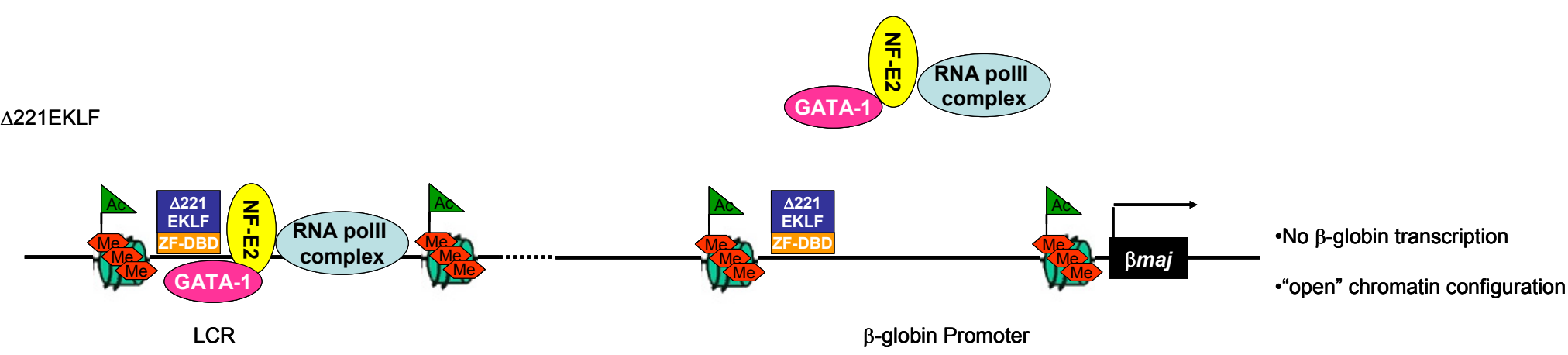

C. EKLF-null

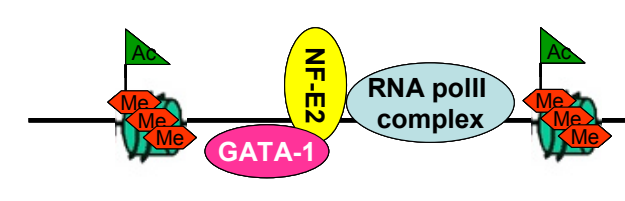

LCR
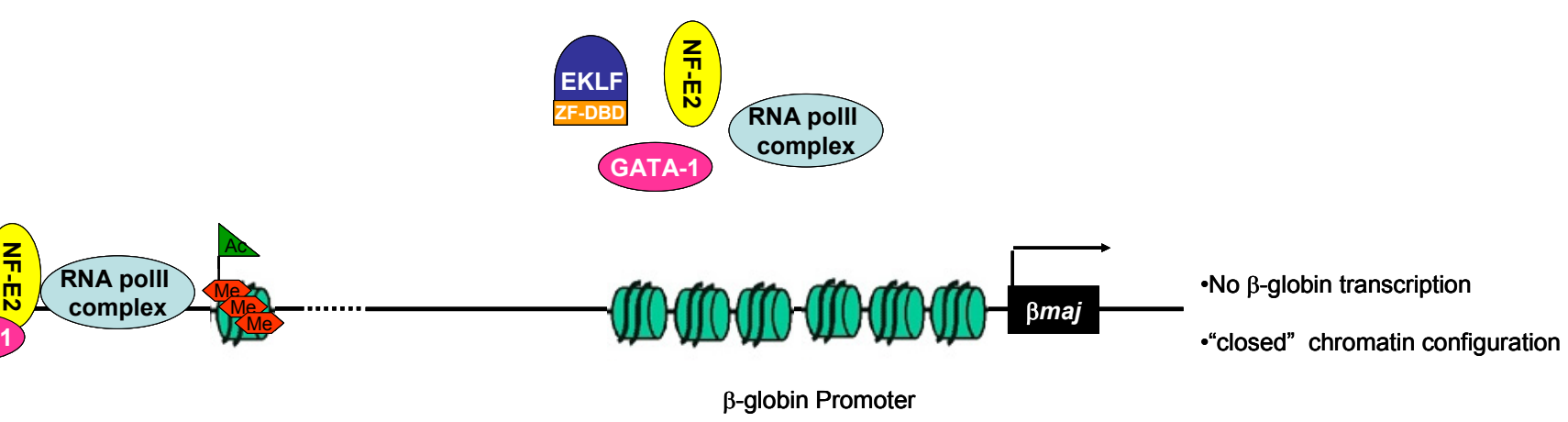


\section{CHAPTER 4: EXPRESSION OF $\triangle 221 E K L F$ RESULTS IN ALTERED ERYTHROID MATURATION AND BLOCKED MEGAKARYOCYTIC EXPANSION}

\section{INTRODUCTION}

Hematopoiesis is a complex developmental process in which hematopoietic stem cells (HSCs) continuously generate all blood cell lineages. The current model of hematopoeisis proposes that HSCs proceed through multiple steps of committed progenitors to give rise to mature blood cells in each lineage (Weissman et al., 2001, Orkin \& Zon, 2008) (see Figure1.1 in Chapter 1). This model is based historically on the results from in vitro assays developed to quantify multi-potential progenitors and lineagerestricted progenitors of bone marrow cells cultured in semi-solid media (Nakorn et al., 2003). Erythroid and megakaryocytic lineages are believed to be derived, at least in part, from a common bipotential progenitor. Termed the megakaryoctye-erythrocyte progenitor (MEP) this cell generates unipotential erythroid or megakaryocytic progenitors (Debili et al., 1996; Nakorn et al., 2003). These precursors express many hematopoietic transcription factors that are essential for both erythropoiesis and megakaryopoiesis. Although many of these factors are essential, the precise mechanism of action/pathways directing the fate of the MEP towards one lineage over another remains obscure.

Two factors, EKLF and Fli-1, have emerged in recent studies as key players in the erythroid-megakaryocytic differentiation switch. Both are expressed in the bipotential MEP (Frontelo et al., 2007); however, the levels of these genes are differentially expressed depending on the commitment fate of the MEP. During early MEP differentiation, EKLF and Fli-1 have contrasting levels of expression. In erythroid progenitors, EKLF expression is high, whereas in megakaryocytic progenitors Fli-1 expression is more than 60 -fold greater compared with erythroid progenitors (Frontelo et al., 2007).

EKLF and Fli-1 protein-protein interactions have also been noted (Starck et al., 2003). These in vitro studies suggest that EKLF and Fli-1 do not inhibit each other's binding activity and Fli-1 mediated activation of a megakaryocytic gene promoter construct (i.e., GpIX) can be repressed by EKLF zinc finger domain alone (Starck et al., 2003). Supporting these observations, Bouilloux et al. (2008) have shown that knockdown of EKLF by shRNA results in an increase in megakaryocytic gene expression in differentiated MEL cells. Furthermore, ChIP analysis revealed increased acetylation of histone H3 and occupancy of PolII, GATA-1, and Fli-1 at megakaryocytic gene promoters with EKLF knock-down in MEL cells. By contrast, studies by Siatecka et al. (2007) propose that sumoylation of lysine 74 of EKLF is critical for repression of megakaryopoiesis. Taken together, these studies suggest three potential, and nonexclusive, models of EKLF-mediated repression of megakaryopoiesis (Figure 4.1): (i) EKLF inhibits Fli-1 gene transcription; (ii) EKLF inhibits Fli-1 binding to its target promoters (i.e. GpIX) by protein-protein interaction; and (iii) EKLF binds to Fli-1- 


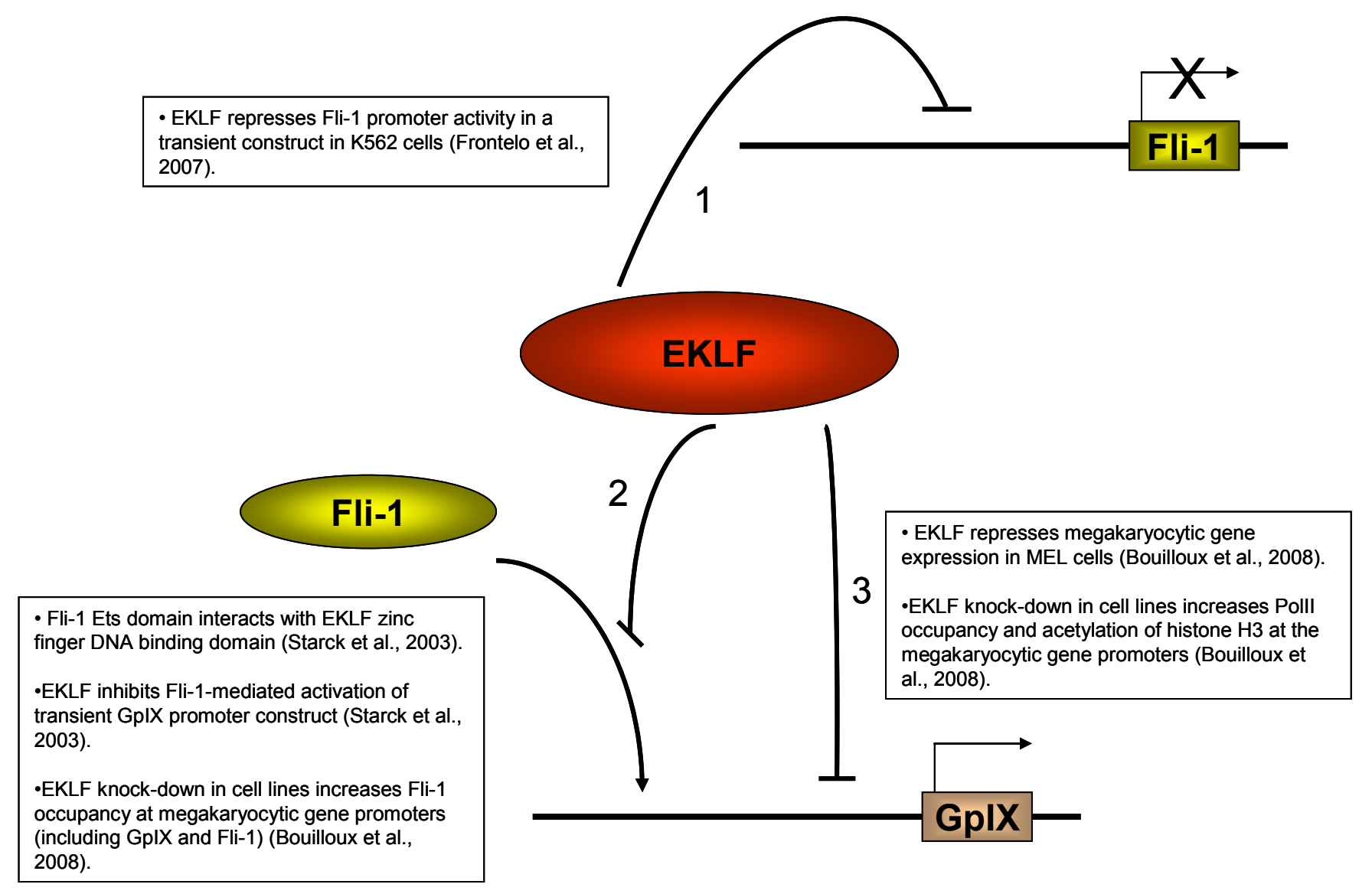

Figure 4.1. Potential models of EKLF-mediated repression of megakaryopoiesis. Shown here is a simplified diagram depicting three possible mechanisms for EKLF-mediated repression of megakaryopoiesis with support for each model from the current literature. (1) EKLF inhibits Fli-1 gene transcription. (2) EKLF inhibits Fli-1 binding to its targets (i.e. GpIX) by protein-protein interaction. (3) EKLF binds to Fli-1-targeted regulatory sequences and blocks Fli-1-medication activation. 
targeted regulatory sequences and blocks Fli-1 mediated activation.

While previous studies have provided significant insights into the role of EKLF in the erythroid-megakaryocytic differentiation program, it is important to note that these studies have been performed in cell lines and may not recapitulate events in vivo. The studies in this chapter seek to expand our current knowledge on the role of EKLF in MEP differentiation utilizing the newly generated mice expressing $\triangle 221 \mathrm{EKLF}$. I report here that, similar to complete loss of EKLF, expression of $\triangle 221 \mathrm{EKLF}$ results in a block of terminal erythroid differentiation at the proerythroblast stage, due in part, to decreased expression of genes essential for erythroid development. However, proliferation of erythroid progenitors is unaltered when compared to wild-type erythroblasts.

In contrast, expression of $\triangle 221 \mathrm{EKLF}$ inhibits the expansion of megakaryocytic progenitors observed with complete loss of EKLF. Molecular analysis of the Fli-1 promoter is consistent with the idea that inhibition of megakaryocytic expansion is achieved, in part, by $\triangle 221 \mathrm{EKLF}$ repression of Fli-1 through recruitment of a Sin3Acontaining repressor complex. Taken together, my results suggest that the $\triangle 221 \mathrm{EKLF}$ domain alone is sufficient for EKLF-dependent inhibition of megakaryocytic differentiation in vivo.

\section{MATERIALS AND METHODS}

\section{Mice}

EKLF-null mice were previously generated via gene targeting by Perkins et al. (1995). $\Delta 221 \mathrm{EKLF}$ mice were generated via gene targeting as described in Chapter 2 of this dissertation. Genotyping of wild-type, EKLF-null, $\triangle 221 \mathrm{EKLF}$ embryos were performed by PCR method as described in Chapter 3.

\section{Colony-forming assays}

Fetal livers cells were obtained by passing E14.5 fetal liver through a $70 \mu \mathrm{m}$ cell strainer in D10 (DMEM + 10\% FBS). To evaluate the erythroid colonies (burst forming units- erythroid; BFU-E), granulopoietic colonies (colony forming units-granulocytemacrophage; CFU-GM), and colonies containing multiple lineages of cells (colonyforming unit-granulocyte-erythroid-monocyte-megakaryocyte; CFU-GEMM), fetal liver cell suspensions were plated $\left(1 \times 10^{4}\right.$ cells $/ \mathrm{mL}$ ) on methylcellulose medium (MethoCult 3434; StemCell Technologies) supplemented with stem cell factor, IL-3, IL-6, and erythropoietin as recommended by the manufacturer. Cells were plated in $35 \mathrm{~mm}$ dishes and maintained at $37^{\circ} \mathrm{C}$ in a humidified atmosphere at $5 \% \mathrm{CO}_{2}$ for $10-12$ days. The colonies were then counted under an inverted microscope and expressed as average number of CFU colonies per culture. Colonies were identified as described in the technical manual for Mouse Colony-Forming Assay using Methocult 
(http://www.stemcell.com/technical/28405_methocult\%20M.pdf).

To evaluate the colony forming units-megakaryocytes (CFU-Mk), fetal liver cell suspensions were plated $\left(1 \times 10^{5}\right.$ cells $\left./ \mathrm{mL}\right)$ on collagen medium (Megacult-C, StemCell Technologies) containing collagen $(1.1 \mathrm{mg} / \mathrm{mL})$, recombinant thrombopoietin $(50 \mathrm{ng} / \mathrm{Ml})$, recombinant IL-3 $(10 \mathrm{ng} / \mathrm{mL})$, recombinant IL-6 $(20 \mathrm{ng} / \mathrm{mL})$, and recombinant IL-11 (50 $\mathrm{ng} / \mathrm{mL}$ ). Cells were plated in double chamber slides in a $100 \mathrm{~mm}$ petri dish and maintained at $37^{\circ} \mathrm{C}$ in a humidified atmosphere at $5 \% \mathrm{CO}_{2}$ for 14 days as recommended by the manufacturer (StemCell Technologies). The slides were fixed with methanolacetone solution after removing the double chamber and stored at $-20^{\circ} \mathrm{C}$ until staining could be performed. Histochemical staining was performed with acetylthiocholiniode (Sigma). The colonies were then counted under an inverted microscope and expressed as average number of CFU-Mk colonies per culture. CFU-Mk colonies were identified as a group of 3 to 50 megakaryocytes per colony with acetyl cholinesterase activity, evident by brown granules in the cytoplasm. Each experiment was performed a minimum of four times, and the data were analyzed using a Student $t$-test.

\section{FACS analysis}

Fetal livers from E14.5 embryos were passed through a 23-gauge needle three times, filtered through a $70 \mu \mathrm{M}$ cell strainer, washed with D10 medium (DMEM $+10 \%$ FBS), and submitted on ice to the Flow Cytometry and Cell Sorting Shared Resource Center at St Jude Children's Research Hospital. Cells were washed in PBS + 5\% FBS and incubated on ice in human gamma gobulin solution $(100 \mathrm{mg} / \mathrm{mL}$ in PBS-5\%) for blocking of nonspecific staining. Cells were then washed in PBS-5\% and incubated on ice for $30 \mathrm{~min}$ with $0.5 \mu \mathrm{g}$ of phycoerythrin (PE)-conjugated anti-mouse CD71 and allophycocyanin (APC)-conjugated anti-mouse TER119 antibodies (BD PharMingen). Results were analyzed using Flow-Jo software (Tree Star).

\section{Globin gene expression by real time RT-PCR}

Real time RT-PCR to analyze the expression of EKLF target genes were performed as described in Chapter 3. The primers used for real time RT-PCR are shown in Appendix Table A.5. Each sample was run in duplicate with at least five independent experiments performed.

\section{Chromatin immunoprecipitation assays}

ChIP assay was performed as described in Chapter 3 with addition of anti-Sin3A (Santa Cruz, sc-994x) antibody. Primers for ChIP analysis at Fli-1 promoter are: forward: CGTGGACCCCGTCATTGTT and reverse: GCACTGCGCACACAGGATACT (Frontelo et al., 2007). Primers for $\beta$-globin promoter are the same as those described in Chapter 3. 


\section{RESULTS}

\section{Expression of $\triangle 221 E K L F$ alters erythroid differentiation and inhibits expansion of megakaryocytic progenitors}

To assess the maturation and differentiation potential of fetal liver cells from $\Delta 221 \mathrm{EKLF}$ embryos, I used a flow cytometry assay that allows quantitative evaluation of erythroid differentiation on the basis of expression of the erythroid-specific TER 119 and nonerythroid-specific CD71 (transferrin receptor) cell surface markers. Cells were flow sorted, and a density plot of relative logarithmic fluorescence units for CD71-expressing versus TER19-expressing was generated (Figure 4.2A). According to previous studies, cells are defined by a characteristic pattern of CD71 and TER119 staining such that CD $71^{\text {med }}$ TER $119^{\text {low }}$ cells are predominantly progenitor cells, CD $71^{\text {high }}$ TER $119^{\text {low }}$ are early basophilic proerythroblasts, CD $71^{\text {high }}$ TER $119^{\text {high }}$ are early and late basophilic erythroblasts, CD $71^{\text {med }}$ TER $119^{\text {high }}$ are chromatophilic and orthochromatophilic erythroblasts, and CD $71^{\text {low }}$ TER $119^{\text {high }}$ are late orthochromatophilic erythroblasts and recticulocytes (Socolovsky et al., 2001; Zhang et al., 2003). Using this assay, I validated previous studies that demonstrated TER119 was absent in EKLF-null fetal liver cells while CD71 expression at the cell surface was normal (Figure 4.2A, top panels) (Hodge et al., 2005), suggesting that loss of EKLF resulted in a block of erythroid maturation at the proerythroblast stage (Pilon et al., 2008). As shown in Figure 4.2A (bottom panels), a comparable pattern of CD71/TER119 staining was observed in fetal liver cells from $\triangle 221 E K L F$ embryos of a similar chronology. Fetal liver cells from EKLF heterozygous and $\triangle 221 E K L F$ heterozygous littermates demonstrated proper execution of the terminal erythroid differentiation program. Thus, it appears that expression of the $\Delta 221 \mathrm{EKLF}$ domain alone is insufficient for execution of the terminal erythroid differentiation program in vivo, resulting in a block at an early stage of erythroid differentiation.

A block in terminal erythroid differentiation as a consequence of $\triangle 221 \mathrm{EKLF}$ expression led me to speculate that $\triangle 221 \mathrm{EKLF}$ affected the proliferation of hematopoietic progenitors in vivo. To quantify the number of erythroid colonies, fetal liver cells from wild-type, $\triangle 221 \mathrm{EKLF}$, and EKLF-null embryos were cultured in a methylcellulose-based medium supplemented with cytokines to promote the growth of BFU-E, CFU-GM, and CFU-GEMM colonies. As shown in Figure 4.2B, there was no statistical difference in the number of BFU-e and CFU-GM colonies cultured from fetal liver of wild-type, $\triangle 221 \mathrm{EKLF}$, and EKLF-null embryos. Analysis of the BFU-E colonies revealed colonies of similar size (Appendix Figure A.3). These observations are in agreement with previous studies in EKLF-null mice (Perkins et al., 1995), consistent with the idea that expression of $\triangle 221 \mathrm{EKLF}$ does not affect the proliferation of erythroid progenitors. On the other hand, these studies revealed comparable numbers of CFUGEMM colonies in $\triangle 221 \mathrm{EKLF}$ and wild-type animals. In contrast, there is an expansion of CFU-GEMM colonies in EKLF-null animals. This observation was quite interesting given that recent studies by Frontelo et al. (2007) and Bouilloux et al. (2008) proposed a novel role for EKLF in megakaryopoiesis. Subsequently, I speculated whether the $\Delta 221 \mathrm{EKLF}$ domain alone is sufficient to inhibit the expansion of megakaryocytic 

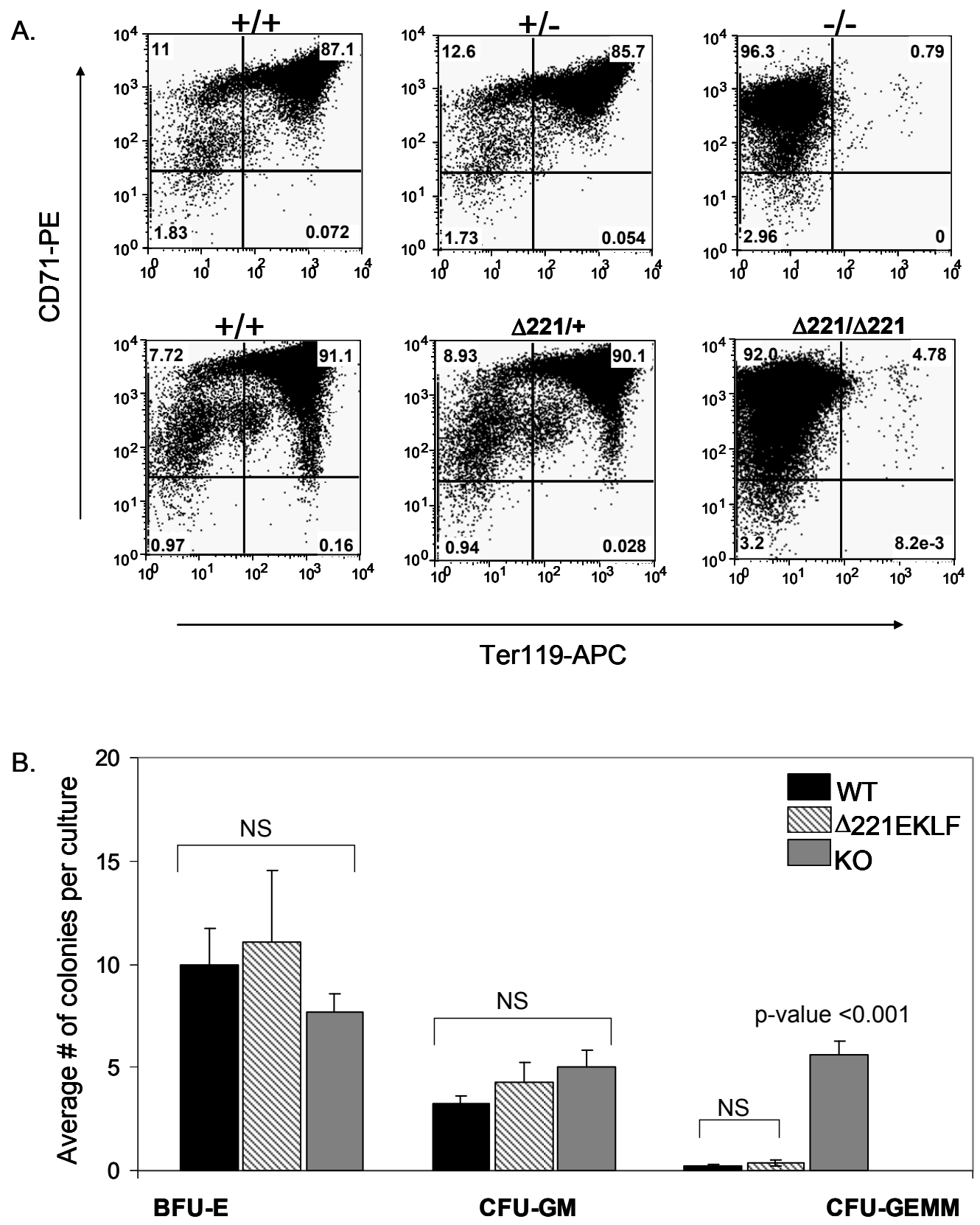

Figure 4.2. Effects of $\Delta 221 \mathrm{EKLF}$ expression on terminal erythroid differentiation and proliferation of fetal liver progenitor cells. (A) Fluorescence-activated cell sorting analysis of E14.5 fetal liver cells from wild-type, EKLF-null heterozygous and EKLFnull embryos (top panel). In a separate experiment, FACS analysis of E14.5 fetal liver from wild-type, $\triangle 221 \mathrm{EKLF}$ heterozygous, and $\triangle 221 \mathrm{EKLF}$ embryos. Cells were stained with anti-CD71 and anti-TER119 antibodies. (B) Analysis of hematopoietic colonyforming cells in E14.5 fetal liver cells from wild-type, $2221 \mathrm{EKLF}$, and EKLF-null embryos. Data shown are averages of at least three independent experiments performed in duplicate (mean $\pm \mathrm{SEM}$ ). $\mathrm{NS}=$ not significant; $p$-value for significant difference shown. 
progenitors observed with complete loss of EKLF.

\section{$\Delta 221 E K L F$ alters expression of non- $\beta$-globin-like erythroid-specific genes}

To determine if the failure of terminal erythroid differentiation in $\triangle 221 \mathrm{EKLF}$ mice was due in part to lack of expression of non- $\beta$-globin erythroid-specific genes, I examined the expression of genes that have been shown to be direct targets of EKLF activation and those that are putative targets (Figure 4.3). Real time RT-PCR analysis revealed that expression of known EKLF target genes, Epb4.9 (Hodge et al., 2005) ankyrin and band 3 (Nilson et al., 2006), BKLF (Funnell et al., 2006), and AHSP (Pilon et al., 2006) were significantly reduced in fetal liver erythroblasts from $\triangle 221 \mathrm{EKLF}$ embryos as compared to wild-type erythroblasts of similar chronology (Figure 4.3A). Similarly, expression of $\triangle 221 \mathrm{EKLF}$ could not rescue high level expression of genes involved in heme biosynthesis (Figure 4.3B). Interestingly, these genes have not been shown to be direct EKLF target genes. These results are consistent with earlier observations at the $\beta$-globin locus (see Figure 3.10), confirming that a larger amino terminal domain of EKLF is required for EKLF-dependent gene transcription in vivo.

\section{A role for $\triangle 221 \mathrm{EKLF}$ in megakaryopoiesis and regulation of Fli-1}

While it is interesting that $\triangle 221 \mathrm{EKLF}$ fails to rescue expression of other non- $\beta$ globin-like erythroid-specific genes in vivo and the local chromatin structure at these gene promoters remains to be analyzed, I have decided to shift my focus to explore further the novel observation that $\triangle 221 \mathrm{EKLF}$ inhibits expansion of megakaryocytic progenitors in vivo. Around the time that I made this observation in $\triangle 221 \mathrm{EKLF}$ mice, two interesting studies utilizing cell lines concluded that EKLF represses megakaryocytic differentiation, at least in part, through Fli-1 repression (Frontelo et al., 2007; Bouilloux et al., 2008). Extending these studies, Siatecka et al. (2007) proposed a critical role for the sumoylation of lysine 74 in repression of megakaryopoiesis in a transgenic mouse model. These studies are reminiscent of earlier studies in which overexpression of EKLF transgene resulted in reduced platelet counts in adult mice (Tewari et al., 1998). While these studies have significantly expanded our knowledge on the role of EKLF in megakaryopoiesis, they have all been studied by overexpression of EKLF protein. Thus, understanding EKLF repression of Fli-1 is of crucial importance, and the $\triangle 221 \mathrm{EKLF}$ mice provide a unique opportunity in which to explore this novel role for EKLF in vivo.

To confirm that the blocked expansion of CFU-GEMM, a mixed cell colony containing granulocyte-erythrocyte-monocyte-megakaryocyte progenitors, was indeed due to inhibition of megakaryocytic progenitors, I performed a more specific assay to identify only CFU-MK by staining for acetylcholinesterase activity. Acetylcholinesterase activity is a marker for murine megakaryocytes (Saleque et al., 2002) and is evident by dark-brown to dark-red granules. As expected, expression of $\triangle 221 \mathrm{EKLF}$ in vivo, resulted in fewer megakaryocytic colonies as compared to complete loss of EKLF (Figure 4.4). More importantly, the number of MK progenitors in $\triangle 221 \mathrm{EKLF}$ fetal liver is equivalent. 
A.

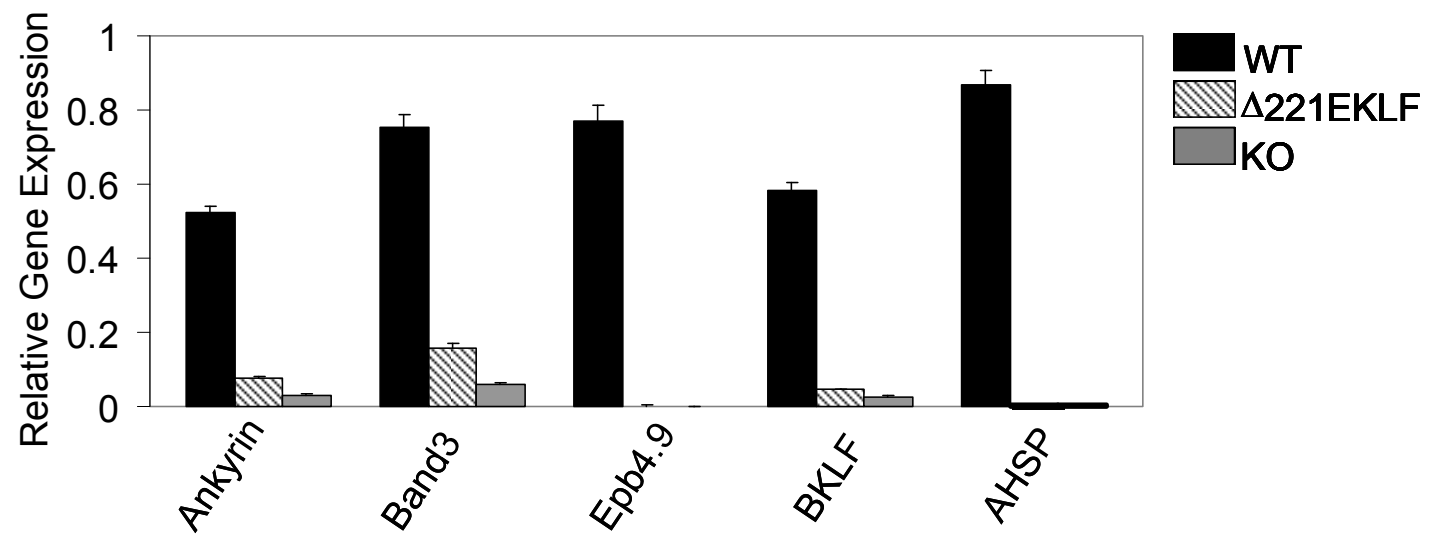

B.

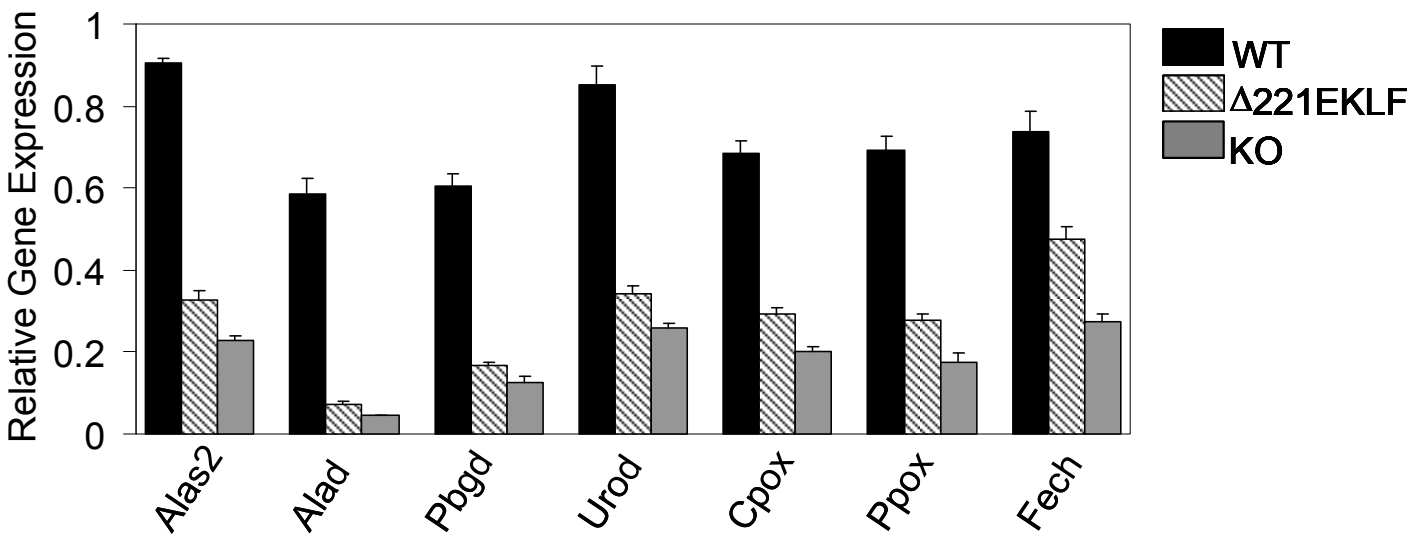

Figure 4.3. Altered pattern of erythroid-specific gene expression in $\triangle 221 E K L F$ fetal liver erythroblasts. cDNA was synthesized from RNA extracted from fetal liver of E14.5 wild-type, $\triangle 221 \mathrm{EKLF}$, and EKLF-null embryos and analyzed by real time RTPCR. (A) Expression level of genes previously shown to be direct targets of EKLFmediated activation. (B) Expression level of genes involved in the heme biosynthesis and not known to be direct targets of EKLF-mediated activation. Data shown are averages of at least five independent experiments performed in duplicate (mean $\pm \mathrm{SEM}$ ). 
to that found in the wild-type fetal liver, consistent with the idea that expression of $\triangle 221 \mathrm{EKLF}$ alone is sufficient to restore megakaryocytic progenitors to wild-type levels. Similarly, the MK colonies obtained from $\triangle 221 E K L F$ fetal liver are similar in appearance and size to those from wild-type fetal liver (Figure 4.4, bottom panel). Together, these observations are consistent with the idea that expression $\triangle 221 \mathrm{EKLF}$ alone is sufficient to block the expansion of MK progenitors observed with complete loss of EKLF

To explore the role of $\triangle 221 \mathrm{EKLF}$ in regulating the Fli-1 promoter, real time RTPCR was used to determine the expression of Fli-1 in fetal liver erythroblasts derived from wild-type, $\triangle 221 \mathrm{EKLF}$, and EKLF-null embryos (Figure 4.5). This was contrasted with expression of $\beta$-globin in the same samples (Figure 4.5A). As shown in Figure 4.5B., there was no statistically significant difference in Fli-1 expression as measured by real time RT-PCR. This observation did not agree with previous expression array analysis (Frontelo et al., 2007) and my earlier findings that expansion of the MK progenitors observed with complete loss of EKLF was inhibited by $\triangle 221 \mathrm{EKLF}$ expression (see Figure 4.2B). It is also possible that I did not observe repression of Fli-1 as expected because I had not analyzed the mRNA levels in the context of MK progenitor cells or for other reasons (see Discussion below). To test if Fli-1 levels were differentially expressed during early murine development, Fli-1 mRNA was determined in erythroid cells derived from yolk sac of E9.5 embryos. Interestingly, a 2-fold decrease in Fli-1 mRNA was noted in $\triangle 221$ EKLF and wild-type yolk sac cells (Figure 4.5C) when compared with EKLF-null cells, although the difference in expression only approached statistical significance $(p$-value $=0.07)$.

To further explore the role of $\triangle 221 \mathrm{EKLF}$ in regulation of Fli-1, chromatin immunoprecipitation (ChIP) assay was utilized to analyze $\Delta 221$ EKLF binding, PolII occupancy, and histone $\mathrm{H} 3$ acetylation at the Fli-1 promoter contrasting these observations with studies of factor occupancy at the $\beta$-globin promoter (Figure 4.6A-C). Analysis of factor binding at the promoters revealed a distinct pattern of binding. $\Delta 221$ EKLF occupied the $\beta$-globin and Fli- 1 promoters in a similar fashion (Figure 4.6A), an observation consistent with previous data for wild-type EKLF (Zhou et al., 2006; Frontelo et al., 2007). While binding of PolII protein at the $\beta$-globin promoter was enriched in wild-type fetal liver erythroblasts, there was minimal PolII binding noted in $\triangle 221 \mathrm{EKLF}$ and EKLF-null erythroblasts (Figure 4.6B, left panel). By contrast, there was a 2-fold decrease in PolII recruitment at the Fli-1 promoter in wild-type and $\triangle 221 \mathrm{EKLF}$ erythroblasts (Figure 4.6B, right panel), consistent with the idea that Fli-1 levels are reduced in these cell types. Similarly, the $\beta$-globin promoter is acetylated to comparable levels in wild-type and $\triangle 221 \mathrm{EKLF}$ erythroblasts (Figure 4.6C, left panel). By contrast, the Fli-1 promoter is hypoacetylated in these cell types and hyperacetylated in EKLF-null cells (Figure 4.6C, right panel). 

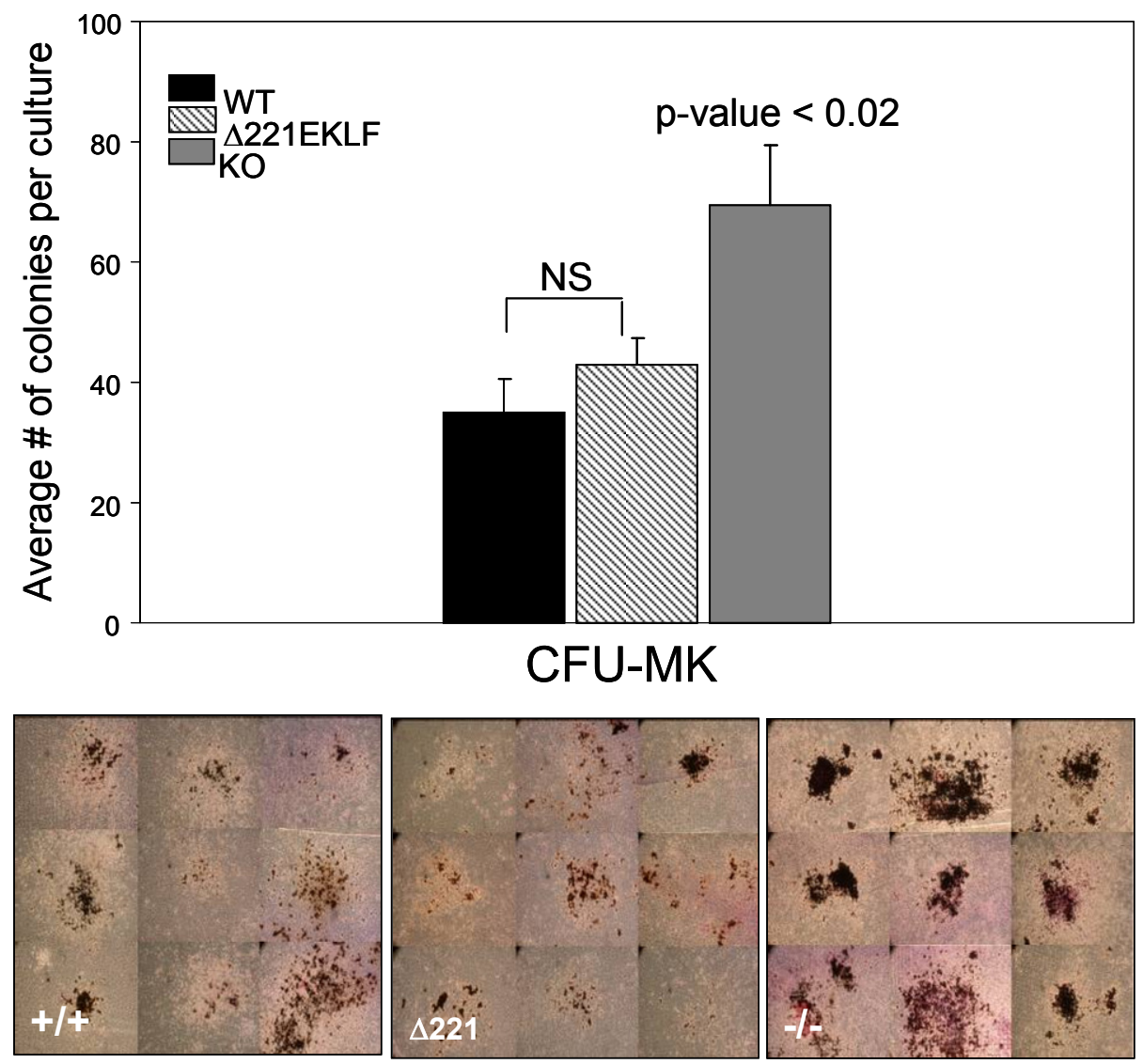

Figure 4.4. Blocked expansion of megakaryocytic progenitors in $\triangle 221 E K L F$ mice. Analysis of megakaryocytic colony-forming cells (CFU-MK) in E14.5 fetal liver cells from wild-type, $\triangle 221 \mathrm{EKLF}$, and EKLF-null embryos. Top panel: frequency of CFU-MK colonies counted per culture condition $\left(1 \times 10^{\wedge 5}\right.$ cells plated). Cells were plated in double chamber slides, maintained at $37^{\circ} \mathrm{C}$ in a humidified atmosphere at $5 \% \mathrm{CO}_{2}$ for 14 days, after which slides were fixed and stained for acetylcholinesterase activity, and CFU-MK counted. Data shown are averages of at least three independent experiments performed in duplicate (mean $\pm \mathrm{SEM}$ ). NS = not significant; $p$-value for significant difference shown. Bottom panel: photographs of representative CFU-MK colonies from wild type, $\triangle 221 \mathrm{EKLF}$, and EKLF-null fetal liver cells after staining. Acetylcholinesterase is evident by the dark-brown granules. 
A.

$\beta$-globin mRNA

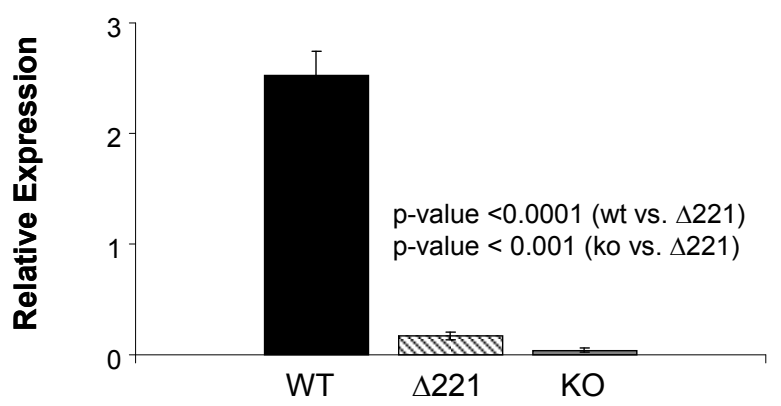

B.

Fli-1 mRNA in E12.5 FL

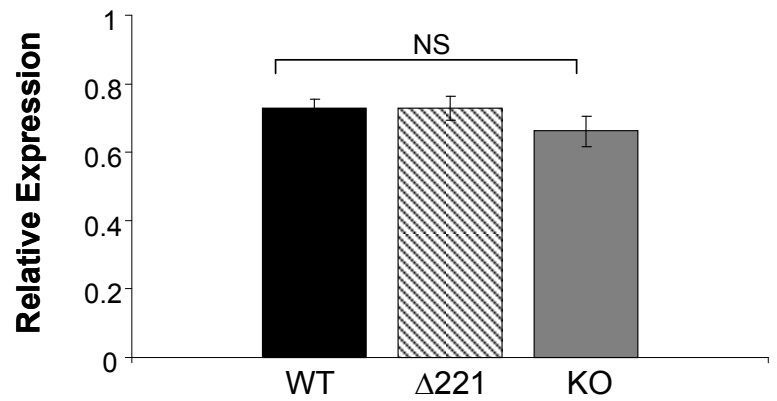

C.

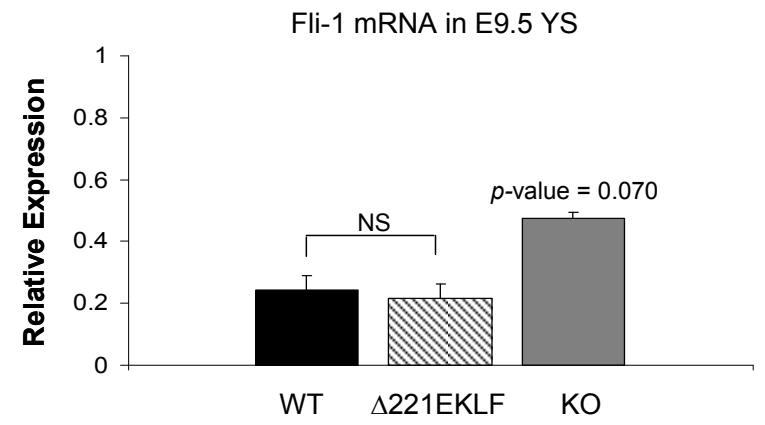

Figure 4.5. Differential expression of $\beta$-globin and Fli-1 gene in $\triangle 221 E K L F$ expressing cells. cDNA prepared from RNA extracted from E14.5 fetal liver (FL) and E9.5 yolk sac (YS) of wild-type, $\triangle 221 \mathrm{EKLF}$, and ELF-null embryos were analyzed by real time RT-PCR for (A) $\beta$-globin mRNA, (B) Fli-1 mRNA in cells derived from fetal liver, and (C) Fli-1 mRNA in cells derived from yolk sac. Data shown as average from at least five independent experiments performed in duplicate (mean $\pm \mathrm{SEM}$ ). $p$-value shown for significant difference. NS = not significant. 
A.

HA- $\Delta$ 221EKLF binding to $\beta$-globin promoter

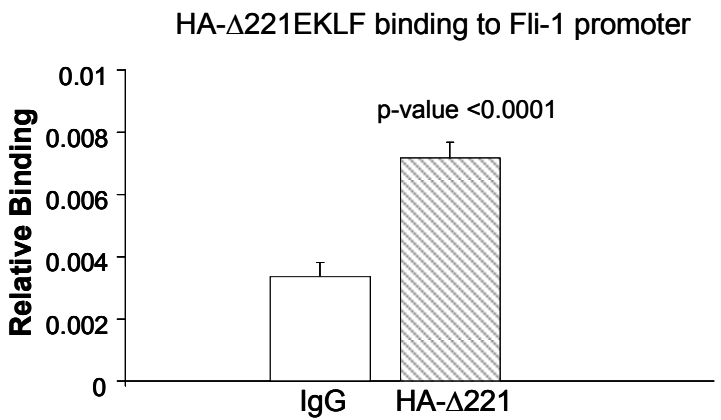

B.

RNA Polll recruitment to $\beta$-globin promoter

RNA Polll recruitment to Fli-1 promoter
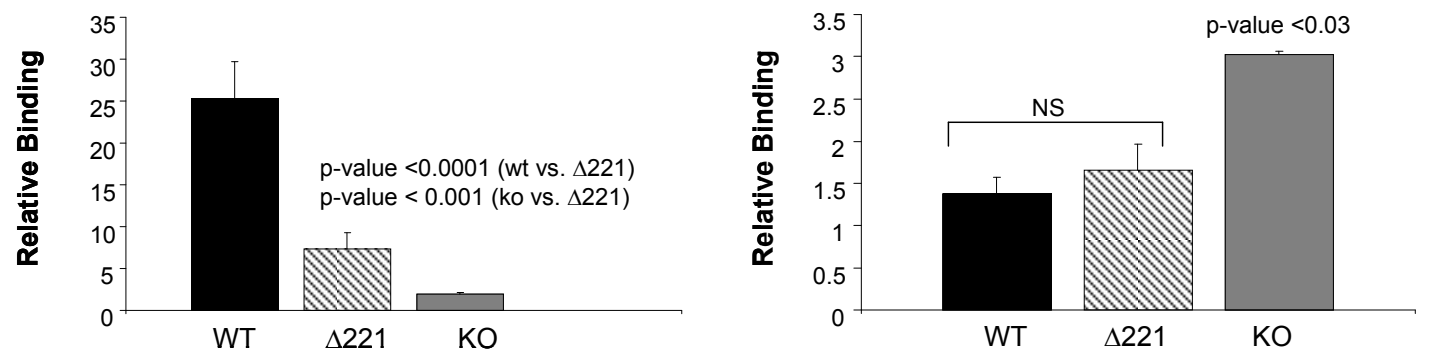

C.

Histone $\mathrm{H} 3$ Acetylation at $\beta$-globin promoter
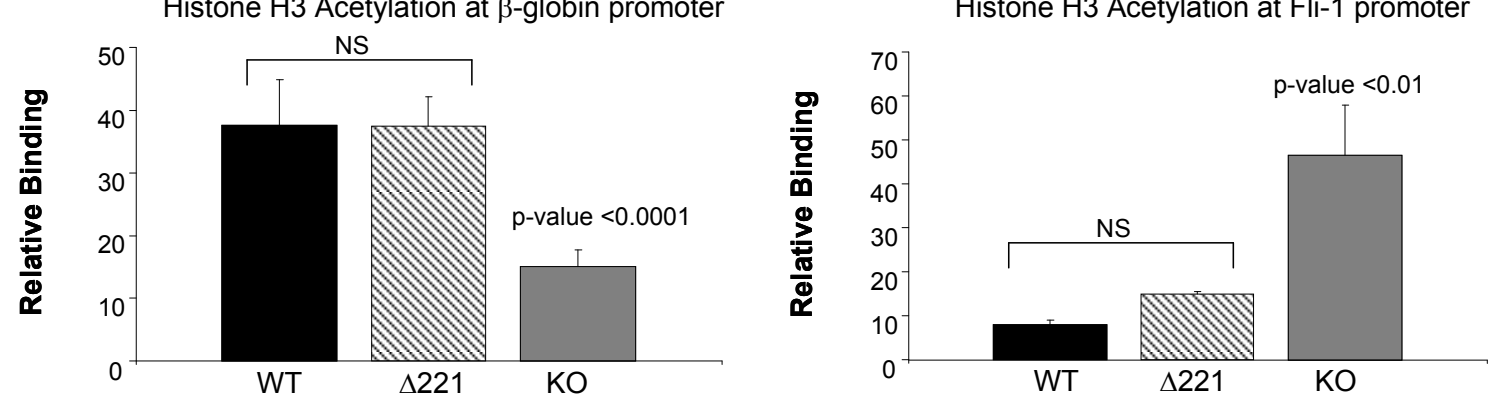

Figure 4.6. Distinct regulation of $\beta$-globin and Fli-1 promoters in $\Delta 221 E K L F$ fetal liver erythroblasts. ChIP assays were utilized to examine factor binding at the $\beta$-globin and Fl-1 promoters, respectively. (A) Binding of $\triangle 221 \mathrm{EKLF}$ at the $\beta$-globin and Fli-1 promoters in fetal liver erythroblasts from E13.5 $\Delta 221 \mathrm{EKLF}$ embryos was detected by anti-HA-specific antiserum. (B) Occupancy of total PolII at the $\beta$-globin and Fli-1 promoters in fetal liver erythroblasts from E13.5 wild-type, $\triangle 221 \mathrm{EKLF}$, and EKLF-null embryos. (C) Acetylation of Histone $\mathrm{H} 3$ at the $\beta$-globin and Fli-1 promoters in fetal liver erythroblasts from E13.5 wild-type $221 \mathrm{EKLF}$, and EKLF-null embryos. Data shown as averages from at least five independent experiments performed in duplicate (mean \pm SEM). $p$-value shown for significant difference. NS $=$ not significant. 


\section{A repressor complex is recruited to the Fli-1 promoter in $\triangle 221 \mathrm{EKLF}$ cells}

To explore the mechanism by which EKLF and $\triangle 221$ EKLF might regulate Fli-1 transcription in vivo, ChIP assays were utilized to examine the recruitment $\operatorname{Sin} 3 \mathrm{~A}$ to the Fli-1 promoter. Previous studies have shown that EKLF exhibits transcriptional repression properties by interacting with Sin3A through its zinc finger DNA binding domain (Chen \& Bieker, 1996; Chen \& Bieker, 2004). Sin3A binding at the Fli-1 promoter was increased 2-fold in fetal liver erythroblasts derived from wild-type and $\triangle 221 \mathrm{EKLF}$ embryos as compared to EKLF-null erythroblasts (Figure 4.7, right panel), thus suggesting that the Fli-1 promoter is repressed. In contrast, no significant binding of Sin3A was noted at the $\beta$-globin promoter (Figure 4.7, left panel), consistent with the idea that a repressor complex has no role in regulation of the $\beta$-globin gene. In summary, these results suggest that EKLF-mediated repression of Fli-1 is achieved in part by recruitment of a Sin3A-containing complex and that expression of $\triangle 221 \mathrm{EKLF}$ alone is sufficient for this recruitment.

\section{DISCUSSION}

Studies in this chapter suggest that expression of $\triangle 221$ EKLF alone is insufficient to support erythroid terminal differentiation; however, this domain of EKLF is sufficient to block expansion of megakaryocytic progenitors in vivo. This novel observation in mice expressing $\triangle 221 \mathrm{EKLF}$ confirms the importance of generating these animals as valuable tools to elucidate the role of EKLF-mediated activation and repression at erythroid- and megakaryocytic-specific gene loci in vivo.

\section{Failure of erythroid differentiation in $\triangle 221 \mathrm{EKLF}$-expressing cells}

EKLF was first characterized as a transcriptional activator that modulated $\beta$ globin gene expression during erythropoiesis. Subsequent studies extended the targets of EKLF-mediated activation to include $\alpha$-hemoglobin stabilizing protein (AHSP), cytoskeleton proteins (ankyrin, Epb4.9), and transcription factors (BKLF) (Pilon et al., 2006; Nilson et al., 2006; Funnell et al.,2007). Understandably, these are critical genes for erythroid development and differentiation. The studies in this chapter not only confirm that EKLF is essential for erythroid gene expression and terminal erythroid differentiation, but also a domain of EKLF ( $\triangle 221 \mathrm{EKLF})$ that is capable of chromatin remodeling (see Chapter 3) is insufficient to fulfill this role in vivo. Indeed, expression of $\triangle 221 E K L F$ in a whole animal model results in a block of terminal erythroid differentiation similar that observed in complete loss of EKLF. However, proliferation of erythroid progenitors is unaffected by expression of $\triangle 221 \mathrm{EKLF}$ in vivo. Furthermore, expression of $\triangle 221 \mathrm{EKLF}$ fails to rescue the expression of ankyrin, band3, Epb4.9, BKLF, AHSP, and all seven genes involved in heme biosynthesis to wild-type levels. It is worth noting that none of the heme biosynthetic genes are known direct targets of EKLF. It would be interesting to speculate on the chromatin structure at these gene promoters; however, studies to examine the DNase I sensitivity at the gene promoters are ongoing in 

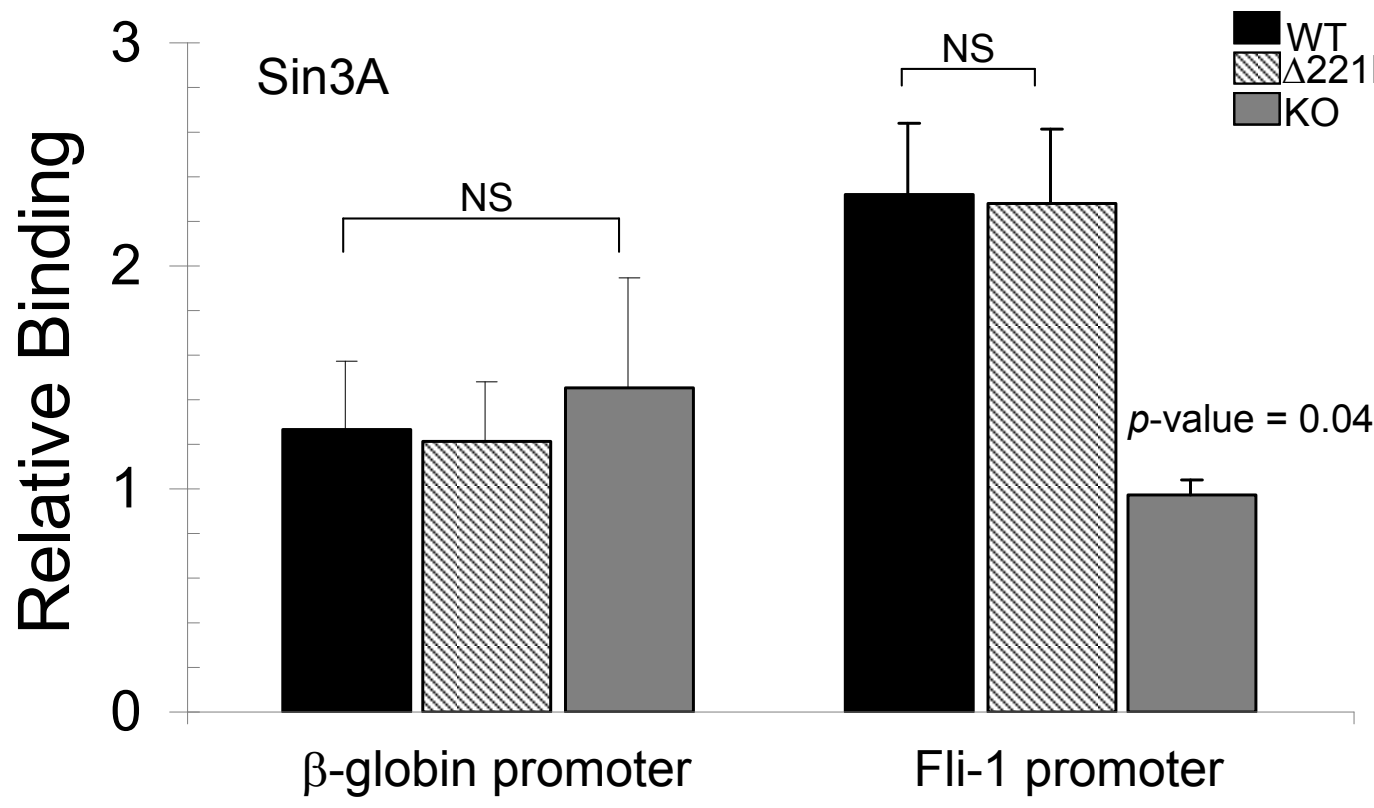

Fli-1 promoter

Figure 4.7. Recruitment of a Sin3A-containing repressor complex. ChIP analysis was performed in fetal liver erythroblasts from E13.5 wild-type, $\triangle 221 \mathrm{EKLF}$, and EKLF-null embryos. Occupancy of Sin3A was determined at Fli-1 promoter contrasted with occupancy at $\beta$-globin promoter. Data shown as averages from at least three independent experiments performed in duplicate (mean $\pm \mathrm{SEM}$ ). $p$-value shown for significant difference. $\mathrm{NS}=$ not significant. 
our laboratory and should provide significant insights into the role of $\triangle 221 \mathrm{EKLF}$ at non$\beta$-globin erythroid-specific gene loci. While it is interesting that $\triangle 221 \mathrm{EKLF}$ is insufficient to rescue non- $\beta$-globin-like erythroid gene expression examined in the present study, the question remains if there are genes that require only the chromatin remodeling domain of EKLF for high-level expression in vivo. Gene expression profiling studies utilizing the $\triangle 221 \mathrm{EKLF}$ mice are ongoing in our laboratory to address this hypothesis.

\section{$\triangle 221 E K L F$ regulates Fli-1 gene and inhibits expansion of megakaryocytic progenitors in vivo}

The most compelling conclusion of the studies presented in this chapter is that expression of $\triangle 221 \mathrm{EKLF}$ is sufficient to repress megakaryocytic differentiation in vivo. This result is particularly interesting given that studies in the previous chapter have shown that $\triangle 221$ EKLF mutant protein is expressed at a lower level than wild-type EKLF protein. Despite this caveat, my studies show that $\triangle 221 \mathrm{EKLF}$ protein is expressed at a sufficient level to repress megakaryopoiesis in vivo. This conclusion is supported by results from colony-forming assays demonstrating equivalent numbers of megakaryocytic progenitors in fetal liver from $\triangle 221 \mathrm{EKLF}$ and wild-type animals (Figure 4.4).

Furthermore, chromatin immunoprecipitation assays show that expression of $\Delta 221 \mathrm{EKLF}$ increases $\triangle 221 \mathrm{EKLF}$ binding to the Fli-1 promoter and decreases PolII occupancy and acetylation of histone $\mathrm{H} 3$ at the Fli-1 promoter (Figure 4.6A-C). Given these results, I would have expected Fli-1 mRNA levels to be reduced in fetal liver erythroblasts from E14.5 wild-type and $\triangle 221$ EKLF embryos as compared to EKLF-null erythroblasts; however, real time RT-PCR analysis showed no significant difference in Fli-1 mRNA levels (Figure 4.5B). Expression arrays between EKLF wild-type and EKLF-null fetal liver cells demonstrate only a 2.5-fold up-regulation of Fli-1 in the absence of EKLF (Frontelo et al., 2007). By contrast, it is possible that the lineage fate decision has already been determined prior to E14.5 of murine development. Thus, Fli-1 mRNA level has been measured in cells that have already committed to the erythroid lineage in $\triangle 221$ EKLF homozygous and wild-type animals. In a similar manner, cells lacking EKLF are driven towards the megakaryocytic lineage. As a result, the level of Fli-1 at this stage of murine development may not play a role in the lineage fate decision. To reconcile this discrepancy, I have also analyzed the levels of Fli-1 mRNA in cells derived from yolk sac of E9.5 of wild-type, $\triangle 221 \mathrm{EKLF}$, and EKLF-null embryos (Figure 4.5C). During this stage of murine development, the yolk sac is the source of all hematopoietic progenitor cells (Palis et al., 1999; Lux et al., 2008). Moreover, hematopoietic progenitor cell types, including erythrocyte and megakaryocyte progenitors, can be cultured from E9.5 yolk sac cells (Rampon \& Huber, 2003). Indeed, I found that Fli-1 mRNA was decreased 2-fold in erythroid cells derived from E9.5 $\triangle 221 \mathrm{EKLF}$ and wild-type yolk sac. Thus, these results are consistent with the idea that the level of Fli-1 is important during early murine development in order to influence lineage fate decisions. 


\section{$\triangle 221 E K L F$ uniquely inhibits Fli-1 by recruitment of a Sin3A-containing corepressor complex}

Previous studies have shown that EKLF can function as a transcriptional repressor through its zinc finger DNA binding domain, which can recruit Sin3A and HDAC1 (Chen \& Bieker, 1996; Chen \& Bieker, 2004) and more recently through sumoylation of lysine 74 , which can then recruit the Mi-2 $\beta$ component of the NuRD repressor complex (Siatecka et al., 2007). Interestingly, in contrast to wild-type EKLF, expression of a K74R EKLF mutant that cannot be sumoylated cannot repress megakaryopoiesis in transgenic mice (Siatecka et al., 2007). Several new results obtained from my studies directly challenge the latter observation. First, I show that expression of $\triangle 221 \mathrm{EKLF}$ is sufficient to repress megakaryocytic differentiation in vivo in a similar manner to wildtype EKLF (Figure 4.4). Thus, this indicates that sumoylation of lysine 74 is not required for EKLF-mediated repression of megakaryopoiesis in vivo (Siatecka et al., 2007). Furthermore, my studies utilized a model in which the $\triangle 221 \mathrm{EKLF}$ protein is expressed from the endogenous EKLF promoter under the control of cis regulatory elements. In contrast, studies by Siatecka et al. (2007) were performed in a transgenic murine model in which the mutant protein was over-expressed under the control of the PF4-gene promoter (Siatecka et al., 2007), which may not recapitulate the events in vivo precisely. The most obvious pitfall is that proteins expressed from heterologous promoters may not be expressed in a tissue- and developmentally-specific manner as the endogenous protein. My studies also found that Sin3A occupancy is increased at the Fli-1 promoter in $\triangle 221 E K L F$ and wild-type erythroblasts (Figure 4.7). These observations are in contrast to studies demonstrating that the NuRD repressor complex is involved in EKLF-mediated repression of megakaryopoiesis (Siatecka et al., 2007). Sin3A is not part of the NuRD complex (Downes et al., 2000). Taken together, my results provide strong support for the $\triangle 221 \mathrm{EKLF}$ domain in EKLF-mediated repression of megakaryocytic differentiation through recruitment of a Sin $3 \mathrm{~A}-$ containing repressor complex.

Three potential models of EKLF-mediated repression of megakaryopoiesis can be derived from review of the recent literature (see Introduction above; Figure 4.1). Although support exists for each model, the precise mechanism by which EKLF represses megakaryocytic differentiation remains to be fully explored. Based on my current studies, I propose a modified model in which EKLF represses megakaryopoiesis, at least in part, by inhibition of Fli-1 through recruitment of a Sin3A-containing repressor complex (Figure 4.8). The $\Delta 221 \mathrm{EKLF}$ domain alone is sufficient for this recruitment. At this time, studies in our laboratory are ongoing to address the other two potential models in the context of $\triangle 221 \mathrm{EKLF}$. We have planned experiments to explore expression of megakaryocytic genes (i.e. GpIX) in vivo and occupancy of $\triangle 221$ EKLF protein and additional factor binding at these gene promoters. Furthermore, we propose studies to confirm EKLF and Fli-1 interaction in vivo by purifying $\triangle 221$ EKLF protein complexes. Regardless of the exact mechanism of EKLF-mediated repression of megakaryopoiesis, the $\triangle 221 \mathrm{EKLF}$ mice have proven to be an indispensable model in which to explore this question in vivo. 


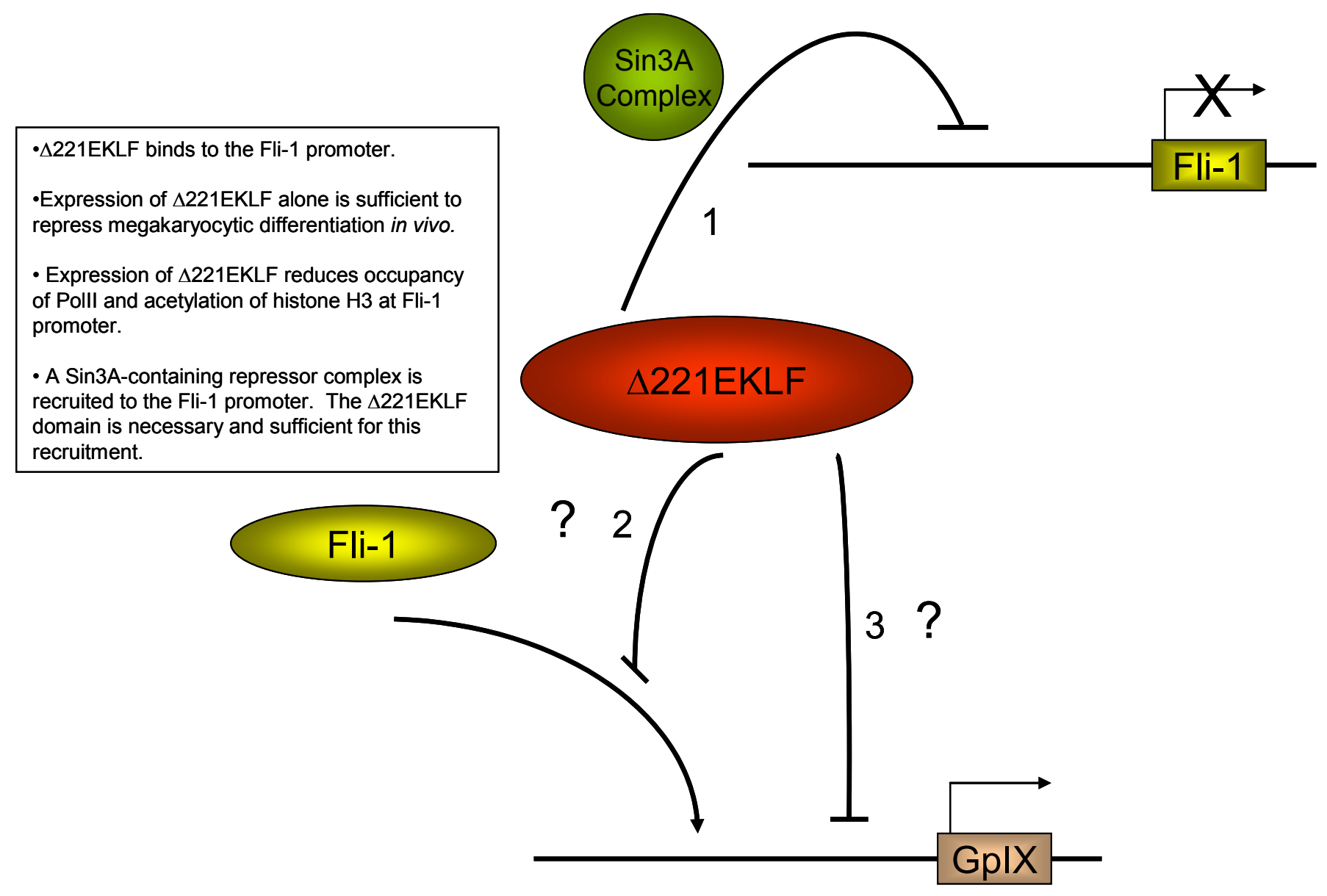

Figure 4.8. Modified potential models of $\triangle 221 \mathrm{EKLF}$-mediated repression of megakaryopoiesis. Shown here is a simplified diagram depicting three possible mechanisms for EKLF-mediated repression of megakaryopoiesis. Results obtained from the current studies in my dissertation support the model in which EKLF inhibits Fli-1 gene transcription through recruitment of a $\operatorname{Sin} 3 \mathrm{~A}$-containing repressor complex. The $\triangle 221 \mathrm{EKLF}$ domain alone is sufficient for this repression in vivo. It is unclear at this juncture how $\triangle 221 \mathrm{EKLF}$ affects the other two potential models of EKLF-mediated repression of megakaryopoiesis. Studies are ongoing to address these questions. 


\section{CHAPTER 5: SUMMARY AND FUTURE DIRECTIONS}

The studies presented in this dissertation contribute to our understanding of the role of the zinc finger transcription factor EKLF or erythroid Krüppel-like factor in erythroid and megakaryocytic differentiation. I have generated mice expressing an amino truncation mutant of EKLF ( $\triangle 221 \mathrm{EKLF})$. This domain of EKLF has been shown to retain full and appropriate chromatin remodeling activity of wild-type EKLF in vivo. However, expression of this domain is insufficient to rescue high-level $\beta$-globin gene transcription in vivo. In contrast, expression of $\Delta 221 \mathrm{EKLF}$ is sufficient to inhibit expansion of megakaryocytic progenitors observed with a complete loss of EKLF. Thus, the studies described in the preceding chapters provided novel and insightful knowledge on the role of EKLF-mediated activation and repression at erythroid and megakaryocytic gene loci.

\section{$\triangle 221 E K L F$ mice are an excellent model to study separable chromatin remodeling and B-globin gene transcription in vivo}

I have generated mice expressing a unique domain of EKLF that uncouples chromatin remodeling and transactivation of $\beta$-globin transcription in an intact animal model. Utilizing these animals, I have shown in Chapter 3 that expression of $\triangle 221 \mathrm{EKLF}$ cannot rescue the EKLF-null phenotype. Similar to EKLF-null mice, the $\Delta 221$ EKLF mice succumb in utero at E15.5 of gestation, partially as a result of severe anemia. This observation is supported by low levels of $\beta$-globin transcripts in $\triangle 221 \mathrm{EKLF}$ erythroblasts. In contrast to lack of EKLF, expression of $\triangle 221 \mathrm{EKLF}$ restores DNase I HS at the $\beta$ globin promoter and induces $\mathrm{H} 3$ acetylation at the $\beta$-globin promoter. Thus, chromatin remodeling and activation at the $\beta$-globin promoter has been uncoupled in vivo. Furthermore, the $\Delta 221 \mathrm{EKLF}$ mice provide a unique opportunity in which to dissect the determinants required for chromatin remodeling and histone modification from those essential for $\beta$-globin gene transcription in vivo.

EKLF has been shown to associate with a SWI/SNF chromatin remodeling complex. EKLF interaction with Brg1, the ATP-catalytic core of the E-RC1, results in chromatin remodeling of a chromatinized $\beta$-globin template (Armstrong et al., 1998; Kadam et al., 2000). Additionally, $2221 \mathrm{EKLF}$ and Brg1 interaction has been confirmed by in vitro studies in our laboratory (Brown et al., 2002). However, evidence for EKLF interaction with $\operatorname{Brg} 1$ at the $\beta$-globin in vivo is still lacking. Thus, the $\triangle 221 \mathrm{EKLF}$ mice are the ideal reagents in which to explore this question in vivo. I propose to immunoprecipitate EKLF and Brg1 in fetal liver erythroblasts to confirm this interaction in vivo. 


\section{What is the role of $\Delta 221 \mathrm{EKLF}$ in erythroid-specific transcription factories?}

Work from Fraser's group has focused on understanding how genes migrate to specialized compartments in the nucleus for transcription called transcription factories (Osborne et al., 2004). These transcription factories are discrete foci containing nascent RNA production and high concentrations of RNA PolII. Using a combination of techniques including three-dimensional (3D) fluorescence in situ hybridization (FISH), immunofluorescence, and chromosome conformation capture (3C) assays, their laboratory has provided support for the existence of such discrete factories. In these studies, they have shown that the $\beta$-globin gene is transcribed in the same factory as AHSP, a gene that is similarly expressed in the erythroid cell. These genes are separated by more than 50 mega bases on murine chromosome 7 . Furthermore, they have shown that genes present on different chromosomes such as the $\beta$ - and $\alpha$-globin genes, also colocalize in the same transcription factory but at a lower frequency than genes linked in cis (Osborne et al., 2004).

The idea of a discrete foci dedicated to transcription is plausible given that the number of factories visible in the nucleus is less than the numbers of expressed genes (Obsorne et al., 2004). However, it is highly tempting to speculate on the factor(s) that recruit the genes to the specialized factories. It would be interesting to determine if EKLF is co-localized with erythroid-specific genes such as AHSP. Our laboratory currently has an on-going collaboration with the Fraser group to address this key question. Furthermore, studies should be extended to include the role of $\triangle 221 \mathrm{EKLF}$ in these transcription factories.

\section{Is $\triangle 221 \mathrm{EKLF}$ sufficient for formation of the Active Chromatin Hub (ACH)?}

High-level expression of the $\beta$-globin genes requires the upstream enhancer LCR. The precise mechanism of how the LCR functions to enhance transcription remains unsettled; however, it is relatively agreed upon that it involves communication between the LCR and the promoter. Indeed, establishment of the chromosome conformation capture or $3 \mathrm{C}$ technique has greatly advanced our knowledge of this communication. Using the $3 \mathrm{C}$ technique, the Grosveld and Fraser groups have determined that the LCR moves in close proximity to actively transcribed genes, forming a structure called the active chromatin hub $(\mathrm{ACH})$. The $\mathrm{ACH}$ is a spatial configuration of the locus in which the LCR loops toward the active $\beta$-globin genes (Tolhuis et al., 2002). Moreover, EKLF, along with GATA-1 but not NF-E2, has been shown to be required for ACH formation (Palstra et al., 2003; Vakoc et al., 2005; Kooren et al., 2007). Thus in the absence of EKLF, a substructure is formed consisting of the regions outside of the locus and part of the LCR but does not contain the genes (Palstra et al., 2003). A recent study from Bresnick's group has demonstrated that the chromatin remodeling factor, Brg1, is required for loop formation between the LCR and the $\beta$-globin promoter (Kim et al., 2009). Despite these advances in our knowledge on LCR/ $\beta$-globin promoter interactions, there are still many unanswered questions. Is chromatin remodeling mandatory for $\mathrm{LCR} / \beta$-promoter interaction via looping? Results from mutant BRG1 mice imply this is a 
requirement (Kim et al., 2009). If yes, I hypothesize that $\triangle 221 \mathrm{EKLF}$ is sufficient to form the $\mathrm{ACH}$ given this domain retains full chromatin remodeling activity. Is active gene transcription an absolute requirement for $\mathrm{ACH}$ formation? If yes, I hypothesize that $\triangle 221 \mathrm{EKLF}$ would be insufficient to establish the ACH given that $\triangle 221 \mathrm{EKLF}$ cannot activate $\beta$-globin gene transcription. Whatever the results, it would be interesting to know the determinants of EKLF required for $\mathrm{ACH}$ formation in vivo.

\section{Does $\Delta 221 E K L F$ regulate other megakaryocytic genes in vivo?}

In Chapter 4, I reported on new and interesting results demonstrating that expression of $\triangle 221 \mathrm{EKLF}$ alone is sufficient to block expansion of megakaryocytic progenitors observed with complete loss of EKLF. This inhibition of megakaryopoiesis occurs, at least in part, by repression of Fli-1, a key regulator of megakaryocytic differentiation. Results from my ChIP analysis of the Fli-1 promoter suggest that $\triangle 221$ EKLF directly regulate Fli-1 expression in vivo through a mechanism involving recruitment of a Sin3A-containing repressor complex to the Fli-1 promoter. These observations are contrasted with previous studies in cell lines that demonstrated sumoylation of EKLF at lysine 74 inhibits megakaryopoiesis through recruitment of a Mi-2 $\beta$-containing complex (Siatecka et al., 2007). It is of interest to note that Mi-2 $\beta$ is not part of any Sin3A-containing corepressor complex (Downes et al., 2000). Thus, my results are consistent with the idea that sumoylation of EKLF is not the only mechanism for inhibition of megakaryopoiesis in vivo, as $\Delta 221 \mathrm{EKLF}$ domain lacks the lysine 74 region.

I presented three potential, but not mutually exclusive, models by which $\triangle 221$ EKLF could inhibit megakaryocytic differentiation. Although my studies have provided support for the model in which $\triangle 221$ EKLF inhibits megakaryopoiesis by directly repressing Fli-1 expression levels in vivo, it would be interesting to explore the other potential models. It is possible EKLF, or $\triangle 221 \mathrm{EKLF}$, could directly regulate other megakaryocytic genes, such as glycoprotein (GP) IX. I propose studies in which the expression of GpIX is determined in $\triangle 221 \mathrm{EKLF}$ erythroblasts compared to wild-type and EKLF-null erythroblasts. I would expect the expression level of GpIX to be reduced in $\triangle 221 \mathrm{EKLF}$ and wild-type erythroblasts as compared to EKLF-null erythroblasts. It is possible we may not see a significant difference in expression of GpIX in wild-type, $\triangle 221 \mathrm{EKLF}$, and EKLF-null cells. Explanations for this observation are i) GpIX is not a direct target of EKLF-mediated repression or ii) GpIX expressions needs to be determined in the context of megakaryocytic progenitors only. Additionally, using ChIP analysis, we should examine the binding of $\triangle 221 \mathrm{EKLF}$ at GpIX promoter. If $\triangle 221 \mathrm{EKLF}$ regulates GpIX, we expect to see $\Delta 221 \mathrm{EKLF}$ protein enrichment at the gene promoter. These future studies would provide significant information on the role of $\triangle 221 \mathrm{EKLF}$ in the regulation of megakaryocytic genes.

Results from in vitro and cellular studies provide evidence for EKLF and Fli-1 interactions, and this protein-protein interaction may play a role in repression of 
megakaryopoiesis. However, evidence for this interaction in vivo is still lacking. It is possible EKLF inhibits megakaryopoiesis by preventing Fli-1 protein binding to its target genes through protein-protein interaction. Studies in MEL cell lines have shown that Fli1 occupancy at megakaryocytic gene promoters is increased with EKLF knock-down. These studies, however, did not confirm EKLF-Fli-1 interaction as the mechanism for this observation. A confirmatory experiment would be to immunoprecipitate $\triangle 221 \mathrm{EKLF}$ and Fli-1 proteins in vivo.

In conclusion, the results in this study provide novel and interesting insights in to the in vivo role of the chromatin remodeling domain of EKLF in definitive hematopoietic cells. The results are consistent with the idea that expression of a truncated $\triangle 221 \mathrm{EKLF}$ protein is sufficient to remodel the local chromatin architecture at the $\beta$-globin promoter; however, expression of the $\triangle 221 \mathrm{EKLF}$ protein alone is not sufficient to transactivate $\beta$ globin gene transcription in vivo. Furthermore, the current study supports a role for $\triangle 221 \mathrm{EKLF}$ in repression of the megakaryocytic progenitor expansion observed with complete loss of EKLF. Future experiments should be directed at exploring the role of $\triangle 221 \mathrm{EKLF}$ in $\beta$-globin LCR/promoter interaction and transcription of erythroid- and megakaryocytic-specific genes. 


\section{LIST OF REFERENCES}

Adelman, C. A., S. Chattopadhyay, and J. J. Bieker. 2002. The BMP/BMPR/Smad pathway directs expression of the erythroid-specific EKLF and GATA1 transcription factors during embryoid body differentiation in serum-free media. Development 129:539-549.

Adolfsson, J., R. Monsson, N. Buza-Vidas, A. Hultquist, K. Liuba, C. T. Jensen, D. Bryder, L. Yang, O. J. Borge, L. A. M. Thoren, K. Anderson, E. Sitnicka, Y. Sasaki, M. Sigvardsson, and S. E. Jacobsen. 2005. Identification of Flt3+ lymphomyeloid stem cells lacking erythro-megakaryocytic potential: a revised road map for adult blood lineage commitment. Cell 121:295-306.

Akashi, K., D. Traver, T. Miyamoto, and I. L. Weissman. 2000. A clonogenic common myeloid progenitor that gives rise to all myeloid lineages. Nature 404:193-197.

Anguita, E., J. Hughes, C. Heyworth, G. A. Blobel, W. G. Wood, and D.R. Higgs. 2004. Globin gene activation during haemopoiesis is driven by protein complexes nucleated by GATA-1 and GATA-2. EMBO J. 23:2841-2852.

Antoniou, M. "Induction of Erythroid-Specific Supression in Murine Erythroleukemia (MEL) Cell Lines." Methods in Molecular Biology: Gene Transfer and Expression Protocols. Ed. E.J.Murray. Clifton: Human Press, 1991. 421-434.

Armstrong, J. A., J. J. Bieker, and B. M. Emerson. 1998. A SWI/SNF-related chromatin remodeling complex, E-RC1, is required for tissue-specific transcriptional regulation by EKLF in vitro. Cell 95:93-104.

Asano, H. and G. Stamatoyannopoulos. 1998. Activation of beta-globin promoter by erythroid Kruppel-like factor. Mol. Cell. Biol. 18:102-109.

Athanasiou, M., G. Mavrothalassitis, L. Sun-Hoffman, and D.G. Blair. 2000. Fli-1 is a suppressor of erythroid differentiation in human hemato-poietic cells. Leukemia 14:435-449.

Baudin, A., O. Ozier-Kalogeropoulos, A. Denouel, F. Lacroute, and C. Cullin. 1993. A simple and efficient method for direct gene deletion in Saccharomyces cerevisiae. Nucl. Acids Res. 21:3329-3330.

Bender, M. A., M. Bulger, J. Close, and M. Groudine. 2000. [beta]-globin gene switching and DNase I sensitivity of the endogenous [beta]-globin locus in mice do not require the locus control region. Mol. Cell 5:387-393.

Bender, T. P. and W. M. Kuehl. 1986. Murine myb protooncogene mRNA: cDNA sequence and evidence for 5 ' heterogeneity. Proc. Natl. Acad. Sci. U. S. A. 83:3204-3208. 
Bernstein, B. E., M. Kamal, K. Lindblad-Toh, S. Bekiranov, D. K. Bailey, D. J. Huebert, S. McMahon, E. K. Karlsson, E. J. Kulbokas III, T. R. Gingeras, S. L. Schreiber, and E. S. Lander. 2005. Genomic maps and comparative analysis of histone modifications in human and mouse. Cell 120:169-181.

Bieker, J.J, L. Ouyang, and X. Chen. 1998. Transcriptional factors for specific globin genes. Ann. N. Y. Acad. Sci. 850:64-69.

Bieker, J. J. 1996. Isolation, genomic structure, and expression of human erythroid Kruppel-like factor (EKLF). DNA Cell. Biol. 15:347-352.

Blobel, G. A. 2002. CBP and p300: versatile coregulators with important roles in hematopoietic gene expression. J. Leukoc. Biol. 71:545-556.

Bouilloux, F., G. Juban, N. Cohet, D. Buet, B. Guyot, W. Vainchenker, F. Louache, and F. Morle. 2008. EKLF restricts megakaryocytic differentiation at the benefit of erythrocytic differentiation. Blood 112:576-584.

Briddell, R. A., J. E. Brandt, J. E. Straneva, E. F. Srour, and R. Hoffman. 1989. Characterization of the human burst-forming unit-megakaryocyte. Blood 74:145151.

Brown, R. C., S. Pattison, J. van Ree, E. Coghill, A. Perkins, S. M. Jane, and J. M. Cunningham. 2002. Distinct domains of erythroid Kruppel-like factor modulate chromatin remodeling and transactivation at the endogenous beta-globin gene promoter. Mol. Cell. Biol. 22:161-170.

Bulger, M. and M. Groudine. 1999. Looping versus linking: toward a model for longdistance gene activation. Genes Dev. 13:2465-2477.

Bultman, S. J., T. C. Gebuhr, and T. Magnuson. 2005. A Brg1 mutation that uncouples ATPase activity from chromatin remodeling reveals an essential role for SWI/SNF-related complexes in $\beta$-globin expression and erythroid development. Genes Dev. 19:2849-2861.

Bungert, J., K. Tanimoto, S. Patel, Q. Liu, M. Fear, and J. D. Engel. 1999. Hypersensitive site 2 specifies a unique function within the human beta -globin locus control region to stimulate globin gene transcription. Mol. Cell. Biol. 19:3062-3072.

Bürgis, S. and A. Gessner. 2007. Unexpected phenotype of STAT6 heterozygous mice implies distinct STAT6 dosage requirements for different IL-4 functions. Int. Arch. Allergy Immunol. 143:263-268.

Chan, J. Y., X. L. Han, and Y. W. Kan. 1993. Isolation of cDNA encoding the human NF-E2 protein. Proc. Natl. Acad. Sci. U. S. A 90:11366-11370. 
Chen, X. and J. J. Bieker. 1996. Erythroid Kruppel-like factor (EKLF) contains a multifunctional transcriptional activation domain important for inter- and intramolecular interactions. EMBO J. 15:5888-5896.

Chen, X. and J. J. Bieker. 2004. Stage-specific repression by the EKLF transcriptional activator. Mol. Cell. Biol. 24:10416-10424.

Cho, Y., S. H. Song, J. J. Lee, N. Choi, C. G. Kim, A. Dean, and A. Kim. 2008. The role of transcriptional activator GATA-1 at human beta-globin HS2. Nucl. Acids Res. $36: 4521-4528$.

Ciavatta, D. J., T. M. Ryan, S. C. Farmer, and T. M. Townes. 1995. Mouse model of human beta zero thalassemia: targeted deletion of the mouse beta maj- and beta min-globin genes in embryonic stem cells. Proc. Natl. Acad. Sci. U. S. A 92:9259-9263.

Coghill, E., S. Eccleston, V. Fox, L. Cerruti, C. Brown, J. Cunningham, S. Jane, and A. Perkins. 2001. Erythroid Kruppel-like factor (EKLF) coordinates erythroid cell proliferation and hemoglobinization in cell lines derived from EKLF null mice. Blood 97:1861-1868.

Copeland, N. G., N. A. Jenkins, and D. Court. 2001. Recombineering: a powerful new tool for mouse functional genomics. Nat. Rev. Genet. 2:769-779.

Cotta-de-Almeida, V., S. Schonhoff, T. Shibata, A. Leiter, and S. B. Snapper. 2003. A new method for rapidly generating gene-targeting vectors by engineering BACs through homologous recombination in bacteria. Genome Res. 13:2190-2194.

Crossley, M., A. P. Tsang, J. J. Bieker, and S. H. Orkin. 1994. Regulation of the erythroid Kruppel-like factor (EKLF) gene promoter by the erythroid transcription factor GATA-1. J. Biol. Chem. 269:15440-15444.

Debili, N., L. Coulombel, L. Croisille, A. Katz, J. Guichard, J. Breton-Gorius, and W. Vainchenker. 1996. Characterization of a bipotent erythro-megakaryocytic progenitor in human bone marrow. Blood 88:1284-1296.

Dekker, J., K. Rippe, M. Dekker, and N. Kleckner. 2002. Capturing chromosome conformation. Science 295:1306-1311.

Deutschbauer, A. M., D. F. Jaramillo, M. Proctor, J. Kumm, M. E. Hillenmeyer, R. W. Davis, C. Nislow, and G. Giaever. 2005. Mechanisms of haploinsufficiency revealed by genome-wide profiling in yeast. Genetics 169:1915-1925.

Dillon, N. and F. Grosveld. 1991. Human gamma-globin genes silenced independently of other genes in the beta-globin locus. Nature 350:252-254. 
Dmitrovsky, E., W. M. Kuehl, G. F. Hollis, I. R. Kirsch, T. P. Bender, and S. Segal. 1986. Expression of a transfected human c-myconcogene inhibits differentiation of a mouse erythroleukaemia cell line. Nature 322:748-750.

Donze, D., T. M. Townes, and J. J. Bieker. 1995. Role of erythroid Kruppel-like factor in human gamma- to beta-globin gene switching. J. Biol. Chem. 270:1955-1959.

Downes, M., P. Ordentlich, H. Y. Kao, J. G. A. Alvarez, and R. M. Evans. 2000. Identification of a nuclear domain with deacetylase activity. Proc. Natl. Acad. Sci. U. S. A. 97:10330-10335.

Drissen, R., M. von Lindern, A. Kolbus, S. Driegen, P. Steinlein, H. Beug, F. Grosveld, and S. Philipsen. 2005. The erythroid phenotype of EKLF-null mice: defects in hemoglobin metabolism and membrane stability. Mol. Cell. Biol. 25:5205-5214.

Drissen, R., R. J. Palstra, N. Gillemans, E. Splinter, F. Grosveld, S. Philipsen, and W. de Laat. 2004. The active spatial organization of the $\beta$-globin locus requires the transcription factor EKLF. Genes Dev. 18:2485-2490.

Dzierzak, E. and A. Medvinsky. 1995. Mouse embryonic hematopoiesis. Trends Genet. 11:359-366.

Eisbacher, M., M. L. Holmes, A. Newton, P. J. Hogg, L. M. Khachigian, M. Crossley, and B. H. Chong. 2003. Protein-protein interaction between Fli-1 and GATA-1 mediates synergistic expression of megakaryocyte-specific genes through cooperative DNA binding. Mol. Cell. Biol. 23:3427-3441.

Engel, J. D. and K. Tanimoto. 2000. Looping, linking, and chromatin activity: new insights into beta-globin locus regulation. Cell 100:499-502.

Enver, T., N. Raich, A. J. Ebens, T. Papayannopoulou, F. Costantini, and G. Stamatoyannopoulos. 1990. Developmental regulation of human fetal-to-adult globin gene switching in transgenic mice. Nature 344:309-313.

Feng, W. C., C. M. Southwood, and J. J. Bieker. 1994. Analyses of beta-thalassemia mutant DNA interactions with erythroid Kruppel-like factor (EKLF), an erythroid cell-specific transcription factor. J. Biol. Chem. 269:1493-1500.

Forrester, W. C., E. Epner, M. C. Driscoll, T. Enver, M. Brice, T. Papayannopoulou, and M. Groudine. 1990. A deletion of the human beta-globin locus activation region causes a major alteration in chromatin structure and replication across the entire beta-globin locus. Genes Dev. 4:1637-1649.

Forsberg, E. C., K. M. Downs, H. M. Christensen, H. Im, P. A. Nuzzi, and E. H. Bresnick. 2000. Developmentally dynamic histone acetylation pattern of a tissue-specific chromatin domain. Proc. Natl. Acad. Sci. U. S. A. 97:14494-14499. 
Friend, C. 1957. Cell-free transmission in adult Swiss mice of a disease having the character of leukemia. J. Exp. Med. 105:307-318.

Frontelo, P., D. Manwani, M. Galdass, H. Karsunky, F. Lohmann, P. G. Gallagher, and J. J. Bieker. 2007. Novel role for EKLF in megakaryocyte lineage commitment. Blood 110:3871-3880.

Fry, C. J. and P. J. Farnham. 1999. Context-dependent transcriptional regulation. J. Biol. Chem. 274:29583-29586.

Fujiwara, Y., C. P. Browne, K. Cunniff, S. C. Goff, and S. H. Orkin. 1996. Arrested development of embryonic red cell precursors in mouse embryos lacking transcription factor GATA-1. Proc. Natl. Acad. Sci. U. S. A. 93:12355-12358.

Funnell, A. P., C. A. Maloney, L. J. Thompson, J. Keys, M. Tallack, A. C. Perkins, and M. Crossley. 2007. Erythroid Kruppel-like factor directly activates the basic Kruppel-like factor gene in erythroid cells. Mol. Cell. Biol. 27:2777-2790.

Greenbaum, D., C. Colangelo, K. Williams, and M. Gerstein. 2003. Comparing protein abundance and mRNA expression levels on a genomic scale. Genome Biol. 4:117.

Grosveld, F., G. B. van Assendelft, D. R. Greaves, and G. Kollias. 1987. Positionindependent, high-level expression of the human beta-globin gene in transgenic mice. Cell 51:975-985.

Hall, M. A., D. J. Curtis, D. Metcalf, A. G. Elefanty, K. Sourris, L. Robb, J. R. Gothert, S. M. Jane, and C. G. Begley. 2003. The critical regulator of embryonic hematopoiesis, SCL, is vital in the adult for megakaryopoiesis, erythropoiesis, and lineage choice in CFU-S12. Proc. Natl. Acad. Sci. U. S. A. 100:992-997.

Hanks, M. C., C. A. Loomis, E. Harris, C. X. Tong, L. Anson-Cartwright, A. Auerbach, and A. Joyner. 1998. Drosophila engrailed can substitute for mouse Engrailed1 function in mid-hindbrain, but not limb development. Development 125:45214530 .

Hanscombe, O., D. Whyatt, P. Fraser, N. Yannoutsos, D. Greaves, N. Dillon, and F. Grosveld. 1991. Importance of globin gene order for correct developmental expression. Genes Dev. 5:1387-1394.

Harju, S., K. J. McQueen, and K. R. Peterson. 2002. Chromatin structure and control of beta-like globin gene switching. Exp. Biol. Med. 227:683-700.

Hart, A., F. Melet, P. Grossfeld, K. Chien, C. Jones, A. Tunnacliffe, R. Favier, and A. Bernstein. 2000. Fli-1 is required for murine vascular and megakaryocytic development and is hemizygously deleted in patients with thrombocytopenia. Immunity 13:167-177. 
Hodge, D., E. Coghill, J. Keys, T. Maguire, B. Hartmann, A. McDowall, M. Weiss, S. Grimmond, and A. Perkins. 2006. A global role for EKLF in definitive and primitive erythropoiesis. Blood 107:3359-3370.

Im, H., J. A. Grass, K. D. Johnson, S. I. Kim, M. E. Boyer, A. N. Imbalzano, J. J. Bieker, and E. H. Bresnick. 2005. Chromatin domain activation via GATA-1 utilization of a small subset of dispersed GATA motifs within a broad chromosomal region. Proc. Natl. Acad. Sci. U. S. A. 102:17065-17070.

Jackers, P., G. Szalai, O. Moussa, and D. K. Watson. 2004. Ets-dependent regulation of target gene expression during megakaryopoiesis. J. Biol. Chem. 279:52183-52190.

Jenkins, N. A., D. J. Gilbert, N. G. Copeland, E. Gruzglin, and J. J. Bieker. 1998. Erythroid Kruppel-like transcription factor (EKLF) maps to a region of mouse chromosome 8 syntenic with human chromosome 19. Mamm. Genome 9:174-176.

Johnson, D., S. W. Horsley, D. M. Moloney, M. Oldridge, S. R. F. Twigg, S. Walsh, M. Barrow, P. R. Njølstad, J. Kunz, G. J. Ashworth, S. A. Wall, L. Kearney, and A. O. M. Wilkie. 1998. A comprehensive screen for TWIST mutations in ptients with craniosynostosis identifies a new microdeletion syndrome of chromosome band 7p21.1. Am. J. Hu. Genet. 63:1282-1293.

Johnson, K. D., H. M. Christensen, B. Zhao, and E. H. Bresnick. 2001. Distinct mechanisms control RNA polymerase II recruitment to a tissue-specific locus control region and a downstream promoter. Mol. Cell 8:465-471.

Johnson, K. D., J. A. Grass, C. Park, H. Im, K. Choi, and E. H. Bresnick. 2003. Highly restricted localization of RNA polymerase II within a locus control region of a tissue-specific chromatin domain. Mol. Cell. Biol. 23:6484-6493.

Kadam, S., G. S. McAlpine, M. L. Phelan, R. E. Kingston, K. A. Jones, and B. M. Emerson. 2000. Functional selectivity of recombinant mammalian SWI/SNF subunits. Genes Dev. 14:2441-2451.

Kaushansky, K. 2008. Historical review: megakaryopoiesis and thrombopoiesis. Blood 111:981-986.

Kawada H, T. Ito, P. N. Pharr, D. D. Spyropoulos, D. K. Watson, and M. Ogawa. 2001. Defective megakaryopoiesis and abnormal erythroid development in Fli-1 genetargeted mice. Int. J. Hematol. 73:463-468.

Keys, J. R., M. R. Tallack, D. J. Hodge, S. O. Cridland, R. David, and A. C. Perkins. 2007. Genomic organisation and regulation of murine alpha haemoglobin stabilising protein by erythroid Kruppel-like factor. Br. J. Haematol. 136:150-157. 
Kiekhaefer, C. M., M. E. Boyer, K. D. Johnson, and E. H. Bresnick. 2004. A WW domain-binding motif within the activation domain of the hematopoietic transcription factor NF-E2 is essential for establishment of a tissue-specific histone modification pattern. J. Biol. Chem. 279:7456-7461.

Kiekhaefer, C. M., J. A. Grass, K. D. Johnson, M. E. Boyer, and E. H. Bresnick. 2002. Hematopoietic-specific activators establish an overlapping pattern of histone acetylation and methylation within a mammalian chromatin domain. Proc. Natl. Acad. Sci. U. S. A. 99:14309-14314.

Kim, S. I. and E. H. Bresnick. 2007. Transcriptional control of erythropoiesis: emerging mechanisms and principles. Oncogene 26:6777-6794.

Kim, S. I., S. J. Bultman, C. M. Kiefer, A. Dean, and E. H. Bresnick. 2009. BRG1 requirement for long-range interaction of a locus control region with a downstream promoter. Proc. Natl. Acad. Sci. U. S. A. 106:2259-2264.

Kingsley, P. D., J. Malik, R. L. Emerson, T. P. Bushnell, K. E. McGrath, L. A. Bloedorn, M. Bulger, and J. Palis. 2006. "Maturational" globin switching in primary primitive erythroid cells. Blood 107:1665-1672.

Kitajima, K., J. Zheng, H. Yen, D. Sugiyama, and T. Nakano. 2006. Multipotential differentiation ability of GATA-1-null erythroid-committed cells. Genes Dev. 20:654-659.

Kooren, J., R. J. Palstra, P. Klous, E. Splinter, M. von Lindern, F. Grosveld, and W. de Laat. 2007. Beta-globin active chromatin hub formation in differentiating erythroid cells and in p45 NF-E2 knock-out mice. J. Biol. Chem. 282:1654416552.

Lakso, M., J. G. Pichel, J. R. Gorman, B. Sauer, Y. Okamoto, E. Lee, F. W. Alt, and H. Westphal. 1996. Efficient in vivo manipulation of mouse genomic sequences at the zygote stage. Proc. Natl. Acad. Sci. U. S. A. 93:5860-5865.

Lee, E., D. Yu, J. Martinez de Velasco, L. Tessarollo, D. A. Swing, D. Court, N. A. Jenkins, and N. G. Copeland. 2001. A highly efficient Escherichia coli-based chromosome engineering system adapted for recombinogenic targeting and subcloning of BAC DNA. Genomics 73:56-65.

Liu, P., N. A. Jenkins, and N. G. Copeland. 2003. A highly efficient recombineeringbased method for generating conditional knockout mutations. Genome Res. $13: 476-484$.

Lohmann, F. and J. J. Bieker. 2008. Activation of Eklf expression during hematopoiesis by Gata2 and Smad5 prior to erythroid commitment. Development 135:20712082. 
Lu, S. J., S. Rowan, M. R. Bani, and Y. Ben-David. 1994. Retroviral integration within the Fli-2 locus results in inactivation of the erythroid transcription factor NF-E2 in Friend erythroleukemias: evidence that NF-E2 is essential for globin expression. Proc. Natl. Acad. Sci. U. S. A. 91:8398-8402.

Lux, C. T., M. Yoshimoto, K. McGrath, S. J. Conway, J. Palis, and M. C. Yoder. 2008. All primitive and definitive hematopoietic progenitor cells emerging before E10 in the mouse embryo are products of the yolk sac. Blood 111:3435-3438.

Mahajan, M. C., S. Karmakar, and S. M. Weissman. 2007. Control of beta globin genes. J. Cell. Biochem. 102:801-810.

Marks, P. A. and R. A. Rifkind. 1988. Hexamethylene bisacetamide-induced differentiation of transformed cells: molecular and cellular effects and therapeutic application. Stem Cells 6:230-240.

Martin, D. I. and S. H. Orkin. 1990. Transcriptional activation and DNA binding by the erythroid factor GF-1/NF-E1/Eryf 1. Genes Dev. 4:1886-1898.

McArthur, M., S. Gerum, and G. Stamatoyannopoulos. 2001. Quantification of DNaseIsensitivity by real-time PCR: quantitative analysis of DNaseI-hypersensitivity of the mouse beta-globin LCR. J. Mol. Biol. 313:27-34.

Miller, I. J. and J. J. Bieker. 1993. A novel, erythroid cell-specific murine transcription factor that binds to the CACCC element and is related to the Kruppel family of nuclear proteins. Mol. Cell. Biol. 13:2776-2786.

Muroya, K., T. Hasegawa, Y. Ito, T. Nagai, H. Isotani, Y. Iwata, K. Yamamoto, S. Fujimoto, S. Seishu, Y. Fukushima, Y. Hasegawa, and T. Ogata. 2001. GATA3 abnormalities and the phenotypic spectrum of HDR syndrome. J. Med. Genet. 38:374-380.

Muyrers, J. P. P., Y. Zhang, and A. F. Stewart. 2001. Techniques: Recombinogenic engineering-new options for cloning and manipulating DNA. Trends Biochem. Sci. 26:325-331.

Nakorn, T. N., T. Miyamoto, and I. L. Weissman. 2003. Characterization of mouse clonogenic megakaryocyte progenitors. Proc. Natl. Acad. Sci. U. S. A. 100:205210.

Navas, P. A., Q. Li, K. R. Peterson, R. A. Swank, A. Rohde, J. Roy, and G. Stamatoyannopoulos. 2002. Activation of the beta-like globin genes in transgenic mice is dependent on the presence of the beta-locus control region. Hum. Mol. Genet. 11:893-903.

Nilson, D. G., D. E. Sabatino, D. M. Bodine, and P. G. Gallagher. 2006. Major erythrocyte membrane protein genes in EKLF-deficient mice. Exp. Hematol. 34:705-712. 
Nuez, B., D. Michalovich, A. Bygrave, R. Ploemacher, and F. Grosveld. 1995. Defective haematopoiesis in fetal liver resulting from inactivation of the EKLF gene. Nature 375:316-318.

Olson, E. N., H.H. Arnold, P. W. J. Rigby, and B. J. Wold. 1996. Know your neighbors: three phenotypes in null mutants of the myogenic bHLH gene MRF4. Cell 85:1-4.

Orkin, S. H., S. E. Antonarakis, and H. H. Kazazian, Jr. 1984. Base substitution at position -88 in a beta-thalassemic globin gene. Further evidence for the role of distal promoter element ACACCC. J. Biol. Chem. 259:8679-8681.

Orkin, S. H. and L. I. Zon. 2008. Hematopoiesis: an evolving paradigm for stem cell biology. Cell 132:631-644.

Osborne, C. S., L. Chakalova, K. E. Brown, D. Carter, A. Horton, E. Debrand, B. Goyenechea, J. A. Mitchell, S. Lopes, W. Reik, and P. Fraser. 2004. Active genes dynamically colocalize to shared sites of ongoing transcription. Nat. Genet. 36:1065-1071.

Ouyang, L., X. Chen, and J. J. Bieker. 1998. Regulation of erythroid Kruppel-like factor (EKLF) transcriptional activity by phosphorylation of a protein kinase casein kinase II site within its interaction domain. J. Biol. Chem. 273:23019-23025.

Palis, J. "Molecular Biology of Erythropoiesis." Molecular Basis of Hematopoiesis. Ed. Amittha Wickrema and Barbara Kee. New York: Springer New York, 2009. 7393.

Palis, J., S. Robertson, M. Kennedy, C. Wall, and G. Keller. 1999. Development of erythroid and myeloid progenitors in the yolk sac and embryo proper of the mouse. Development 126:5073-5084.

Palstra, R. J., B. Tolhuis, E. Splinter, R. Nijmeijer, F. Grosveld, and W. de Laat. 2003. The beta-globin nuclear compartment in development and erythroid differentiation. Nat. Genet. 35:190-194.

Pandya, K., D. Donze, and T. M. Townes. 2001. Novel transactivation domain in erythroid Kruppel-like factor (EKLF). J. Biol. Chem. 276:8239-8243.

Pandya, K. and T. M. Townes. 2002. Basic residues within the Kruppel zinc finger DNA binding domains are the critical nuclear localization determinants of EKLF/KLF1. J. Biol. Chem. 277:16304-16312.

Pazin, M. J., P. Bhargava, E. P. Geiduschek, and J. T. Kadonaga. 1997. Nucleosome mobility and the maintenance of nucleosome positioning. Science 276:809-812.

Pereira R, C. T. Quang, I. Lesault, H. Dolznig, H. Beug, and J. Ghysdael. 1999. FLI-1 inhibits differentiation and induces proliferation of primary erythroblasts. Oncogene. 18:1597-1608. 
Perkins, A. C., A. H. Sharpe, and S. H. Orkin. 1995. Lethal beta-thalassaemia in mice lacking the erythroid CACCC-transcription factor EKLF. Nature 375:318-322.

Perkins, A. C., K. M. Gaensler, and S. H. Orkin. 1996. Silencing of human fetal globin expression is impaired in the absence of the adult beta-globin gene activator protein EKLF. Proc. Natl. Acad. Sci. U. S. A. 93:12267-12271.

Perkins, A. C., K. R. Peterson, G. Stamatoyannopoulos, H. E. Witkowska, and S. H. Orkin. 2000. Fetal expression of a human A-gamma globin transgene rescues globin chain imbalance but not hemolysis in EKLF null mouse embryos. Blood 95:1827-1833.

Perry, C. and H. Soreq. 2002. Transcriptional regulation of erythropoiesis: fine tuning of combinatorial multi-domain elements. Eur. J. Biochem. 269:3607-3618.

Peterson, K. R. and G. Stamatoyannopoulos. 1993. Role of gene order in developmental control of human gamma- and beta-globin gene expression. Mol. Cell. Biol. $13: 4836-4843$.

Peterson, K. R., C. H. Clegg, and Q. Li. 1997. Production of transgenic mice with yeast artificial chromosomes. Trends Genet. 13:61-66.

Pevny, L., C. S. Lin, V. D'Agati, M. C. Simon, S. H. Orkin, and F. Costantini. 1995. Development of hematopoietic cells lacking transcription factor GATA-1. Development 121:163-172.

Pham, C., D. MacIvor, B. Hug, J. Heusel, and T. Ley. 1996. Long-range disruption of gene expression by a selectable marker cassette. Proc. Natl. Acad. Sci. U. S. A. 93:13090-13095.

Phatnani, H. P. and A. L. Greenleaf. 2006. Phosphorylation and functions of the RNA polymerase II CTD. Genes Dev. 20:2922-2936.

Pilon, A. M., D. G. Nilson, D. Zhou, J. Sangerman, T. M. Townes, D. M. Bodine, and P. G. Gallagher. 2006. Alterations in expression and chromatin configuration of the alpha hemoglobin-stabilizing protein gene in erythroid Kruppel-like factordeficient mice. Mol. Cell. Biol. 26:4368-4377.

Pilon, A. M., M. O. Arcasoy, H. K. Dressman, S. E. Vayda, Y. D. Maksimova, J. I. Sangerman, P. G. Gallagher, and D. M. Bodine. 2008. Failure of terminal erythroid differentiation in EKLF-deficient mice is associated with cell cycle perturbation and reduced expression of E2F2. Mol. Cell. Biol. 28:7394-7401.

Pravtcheva D, M. Rabin, M. Bartolomei, J. Corden, F.H. Ruddell. 1986. Chromosomal assignment of gene encoding the largest subunit of RNA polymerase II in the mouse. Somat. Cell Mol. Genet. 12:523-528. 
Quadrini, K. J. and J. J. Bieker. 2002. Kruppel-like zinc fingers bind to nuclear import proteins and are required for efficient nuclear localization of erythroid Kruppellike factor. J. Biol. Chem. 277:32243-32252.

Quadrini, K. J., E. Gruzglin, and J. J. Bieker. 2008. Non-random subcellular distribution of variant EKLF in erythroid cells. Exp. Cell Res. 314:1595-1604.

Quadrini, K. J. and J. J. Bieker. 2006. EKLF/KLF1 is ubiquitinated in vivo and its stability is regulated by activation domain sequences through the $26 \mathrm{~S}$ proteasome. FEBS Lett. 580:2285-2293.

Radhika, S., S. K. Choudhary, L. C. Garg, and A. Dixit. 1995. Induction of differentiation in murine erythroleukemia cells by 1[alpha],25-dihydroxy vitamin D3. Cancer Lett. 90:225-230.

Rampon, C. and P. Huber. 2003. Multilineage hematopoietic progenitor activity generated autonomously in the mouse yolk sac: analysis using angiogenesisdefective embryos. Int. J. Dev. Biol. 47:273-280.

Roebroek, A. J. M., X. Wu, and R. J. Bram. “Knockin Approaches.” Transgenic Mouse. Ed. Marten H. Hofker and Jan van Deursen. Humana Press, 2002. 187-200.

Saleque, S., S. Cameron, and S. H. Orkin. 2002. The zinc-finger proto-oncogene Gfi-1b is essential for development of the erythroid and megakaryocytic lineages. Genes Dev. 16:301-306.

Santos-Rosa, H., R. Schneider, A. J. Bannister, J. Sherriff, B. E. Bernstein, N. C. T. Emre, S. L. Schreiber, J. Mellor, and T. Kouzarides. 2002. Active genes are tri-

Sawado, T., K. Igarashi, and M. Groudine. 2001. Activation of beta-major globin gene transcription is associated with recruitment of NF-E2 to the beta-globin LCR and gene promoter. Proc. Natl. Acad. Sci. U. S. A. 98:10226-10231.

Sawado, T., J. Halow, M. A. Bender, and M. Groudine. 2003. The $\beta$-globin locus control region (LCR) functions primarily by enhancing the transition from transcription initiation to elongation. Genes Dev. 17:1009-1018.

Shivdasani, R. A. and S. H. Orkin. 1995. Erythropoiesis and globin gene expression in mice lacking the transcription factor NF-E2. Proc. Natl. Acad. Sci. U. S. A. $92: 8690-8694$.

Shivdasani, R. A. and S. H. Orkin. 1996. The transcriptional control of hematopoiesis. Blood 87:4025-4039.

Shivdasani, R. A., Y. Fujiwara, M. A. McDevitt, and S. H. Orkin. 1997. A lineageselective knockout establishes the critical role of transcription factor GATA-1 in megakaryocyte growth and platelet development. EMBO J. 16:3965-3973. 
Shivdasani, R. A. 2001. Molecular and transcriptional regulation of megakaryocyte differentiation. Stem Cells 19:397-407.

Shizuya, H., B. Birren, U. J. Kim, V. Mancino, T. Slepak, Y. Tachiiri, and M. Simon. 1992. Cloning and stable maintenance of 300-kilobase-pair fragments of human DNA in Escherichia coli using an F-factor-based vector. Proc. Natl. Acad. Sci. U. S. A. 89:8794-8797.

Shyu, Y. C., S. C. Wen, T. L. Lee, X. Chen, C. T. Hsu, H. Chen, R. L. Chen, J. L. Hwang, and C. K. Shen. 2006. Chromatin-binding in vivo of the erythroid kruppel-like factor, EKLF, in the murine globin loci. Cell Res. 16:347-355.

Siatecka, M., L. Xue, and J. J. Bieker. 2007. Sumoylation of EKLF promotes transcriptional repression and is involved in inhibition of megakaryopoiesis. Mol. Cell. Biol. 27:8547-8560.

Singer, D., M. Cooper, G. M. Maniatis, P. A. Marks, and R. A. Rifkind. 1974. Erythropoietic differentiation in colonies of cells transformed by Friend virus. Proc. Natl. Acad. Sci. U. S. A. 71:2668-2670.

Socolovsky, M., A. E. J. Fallon, and H. F. Lodish. 1998. The prolactin receptor rescues EpoR-/- erythroid progenitors and replaces EpoR in a synergistic interaction with c-kit. Blood 92:1491-1496.

Southwood, C. M., K. M. Downs, and J. J. Bieker. 1996. Erythroid Kruppel-like factor exhibits an early and sequentially localized pattern of expression during mammalian erythroid ontogeny. Dev. Dyn. 206:248-259.

Stamatoyannopoulos, G. 1991. Human hemoglobin switching. Science 252:383.

Starck, J., N. Cohet, C. Gonnet, S. Sarrazin, Z. Doubeikovskaia, A. Doubeikovski, A. Verger, M. Duterque-Coquillaud, and F. Morle. 2003. Functional crossantagonism between transcription factors FLI-1 and EKLF. Mol. Cell. Biol. 23:1390-1402.

Steger DJ and Workman JL. 1996. Remodeling chromatin structures for transcription: What happens to the histones? BioEssays 18:875-884.

Takebayashi, K., K. Chida, I. Tsukamoto, E. Morii, H. Munakata, H. Arnheiter, T. Kuroki, Y. Kitamura, and S. Nomura. 1996. The recessive phenotype displayed by a dominant negative microphthalmia- associated transcription factor mutant is a result of impaired nucleation potential. Mol. Cell. Biol. 16:1203-1211.

Tewari, R., N. Gillemans, M. Wijgerde, B. Nuez, M. von Lindern, F. Grosveld, and S. Philipsen. 1998. Erythroid Kruppel-like factor (EKLF) is active in primitive and definitive erythroid cells and is required for the function of 5'HS3 of the betaglobin locus control region. EMBO J. 17:2334-2341. 
Tober, J., A. Koniski, K. E. McGrath, R. Vemishetti, R. Emerson, K. K. L. de MesyBentley, R. Waugh, and J. Palis. 2007. The megakaryocyte lineage originates from hemangioblast precursors and is an integral component both of primitive and of definitive hematopoiesis. Blood 109:1433-1441.

Tolhuis, B., R. J. Palstra, E. Splinter, F. Grosveld, and W. de Laat. 2002. Looping and interaction between hypersensitive sites in the active [beta]-globin locus. Mol. Cell 10:1453-1465.

Trimborn, T., J. Gribnau, F. Grosveld, and P. Fraser. 1999. Mechanisms of developmental control of transcription in the murine alpha- and beta-globin loci. Genes Dev. 13:112-124.

Tsai, F. Y., C. P. Browne, and S. H. Orkin. 1998. Knock-in mutation of transcription factor GATA-3 into the GATA-1 locus: partial rescue of GATA-1 loss of function in erythroid cells. Dev. Biol. 196:218-227.

Tsai, S. F., D. I. K. Martin, L. I. Zon, A. D. D'Andrea, G. G. Wong, and S. H. Orkin. 1989. Cloning of cDNA for the major DNA-binding protein of the erythroid lineage through expression in mammalian cells. Nature 339:446-451.

Tsang, A. P., J. E. Visvader, C. A. Turner, Y. Fujiwara, C. Yu, M. J. Weiss, M. Crossley, and S. H. Orkin. 1997. FOG, a multitype zinc finger protein, acts as a cofactor for transcription factor GATA-1 in erythroid and megakaryocytic differentiation. Cell 90:109-119.

Tsang, A. P., Y. Fujiwara, D. B. Hom, and S. H. Orkin. 1998. Failure of megakaryopoiesis and arrested erythropoiesis in mice lacking the GATA-1 transcriptional cofactor FOG. Genes Dev. 12:1176-1188.

Vakoc, C. R., D. L. Letting, N. Gheldof, T. Sawado, M. A. Bender, M. Groudine, M. J. Weiss, J. Dekker, and G. A. Blobel. 2005. Proximity among distant regulatory elements at the [beta]-globin locus requires GATA-1 and FOG-1. Mol. Cell 17:453-462.

van Ree, J. H., M. A. Roskrow, A. M. Becher, R. McNall, V. A. Valentine, S. M. Jane, and J. M. Cunningham. 1997. The human erythroid-specific transcription factor EKLF localizes to chromosome 19p13.12-p13.13. Genomics 39:393-395.

Wang, Y., P. N. J. Schnegelsberg, J. Dausman, and R. Jaenisch. 1996. Functional redundancy of the muscle-specific transcription factors Myf5 and myogenin. Nature 379:823-825.

Watson, D. K., F. E. Smyth, D. M. Thompson, J. Q. Cheng, J. R. Testa, T. S. Papas, and A. Seth. 1992. The ERGB/Fli-1 gene: isolation and characterization of a new member of the family of human ETS transcription factors. Cell. Growth Differ. 3:705-713. 
Weiss, M. J. and S. H. Orkin. 1995. Transcription factor GATA-1 permits survival and maturation of erythroid precursors by preventing apoptosis. Proc. Natl. Acad. Sci. U. S. A. 92:9623-9627.

Weissman, I. L., D. J. Anderson, and F. Gage. 2001. Stem and progenitor cells: Origins, phenotypes, lineage commitments, and transdifferentiations. Ann. Rev. Cell Dev. Biol. 17:387-403.

Wijgerde, M., F. Grosveld, and P. Fraser. 1995. Transcription complex stability and chromatin dynamics in vivo. Nature 377:209-213.

Wijgerde, M., J. Gribnau, T. Trimborn, B. Nuez, S. Philipsen, F. Grosveld, and P. Fraser. 1996. The role of EKLF in human beta-globin gene competition. Genes Dev. 10:2894-2902.

Yan, C. and D. D. Boyd. 2006. Histone H3 acetylation and H3 K4 methylation define distinct chromatin regions permissive for transgene expression. Mol. Cell. Biol. 26:6357-6371.

Yu, D., H. M. Ellis, E.-C. Lee, N. A. Jenkins, N. G. Copeland, and D. Court. 2000. An efficient recombination system for chromosome engineering in Escherichia coli. Proc. Natl. Acad. Sci. U. S. A. 97:5978-5983.

Zhang, J., M. Socolovsky, A. W. Gross, and H. F. Lodish. 2003. Role of Ras signaling in erythroid differentiation of mouse fetal liver cells: functional analysis by a flow cytometry-based novel culture system. Blood 102:3938-3946.

Zhang, W., S. Kadam, B. M. Emerson, and J. J. Bieker. 2001. Site-specific acetylation by p300 or CREB binding protein regulates erythroid Kruppel-like factor transcriptional activity via its interaction with the SWI-SNF complex. Mol. Cell. Biol. 21:2413-2422.

Zhang, W. and J. J. Bieker. 1998. Acetylation and modulation of erythroid Krüppel-like factor (EKLF) activity by interaction with histone acetyltransferases. Proc. Natl. Acad. Sci. U. S. A. 95:9855-9860.

Zhang, X. M. and J. D. Huang. 2003. Combination of overlapping bacterial artificial chromosomes by a two-step recombinogenic engineering method. Nucl. Acids Res. 31:e81.

Zhou, D., J. X. Ren, T. M. Ryan, N. P. Higgins, and T. M. Townes. 2004. Rapid tagging of endogenous mouse genes by recombineering and ES cell complementation of tetraploid blastocysts. Nucl. Acids Res. 32:e128.

Zhou, D., K. M. Pawlik, J. Ren, C. W. Sun, and T. M. Townes. 2006. Differential binding of EKLF to embryonic/fetal globin gene promoters during development. J. Biol. Chem. 281:16052-16057. 
Zon, L. I., Y. Yamaguchi, K. Yee, E. A. Albee, A. Kimura, J. C. Bennett, S. H. Orkin, and S. J. Ackerman. 1993. Expression of mRNA for the GATA-binding proteins in human eosinophils and basophils: potential role in gene transcription. Blood 81:3234-3241.

Zon, L. I. 1995. Developmental biology of hematopoiesis. Blood 86:2876-2891. 
APPENDIX: SUPPORTING FIGURES AND TABLES 
Table A.1. Putative EKLF-dependent genes.

\begin{tabular}{|c|c|c|}
\hline \multicolumn{2}{|c|}{ Gene symbol } & \multirow{2}{*}{$\begin{array}{c}\begin{array}{c}\text { Signal log ratio } \\
\text { (null/wt) }\end{array} \\
6.1\end{array}$} \\
\hline SRY-box containing gene 2 & Sox 2 & \\
\hline acetyl-Coenzyme A synthetase 2 & Acas21 & 4.2 \\
\hline calcium channel, voltage-dependent & Cacnalb & 4.0 \\
\hline benzodiazapine receptor associated protein 1 & Bzrap1 & 2.3 \\
\hline inhibitor of DNA binding 4 & $\mathrm{Id} 4$ & 1.7 \\
\hline potassium voltage-gated channel & Kcnq5 & 1.7 \\
\hline erythroid differentiation regulator 1 & Erdr1 & 1.4 \\
\hline eukaryotic translation initiation factor 2 & Eif2s3y & 1.3 \\
\hline insulin-like growth factor binding protein 1 & Igfbp 1 & 1.3 \\
\hline talin 1 & $\mathrm{~T} \ln 1$ & 1.3 \\
\hline erythropoietin receptor & Epor & -1 \\
\hline ankyrin repeat and $\mathrm{BTB}(\mathrm{POZ})$ & Abtb1 & -1.1 \\
\hline eosinophil-associated, ribonuclease & Ear1 & -1.1 \\
\hline myeloblastosis oncogene-like 2 & Mybl2 & -1.1 \\
\hline transportin 2 (importin 3 , karyopherin beta $2 \mathrm{~b}$ ) & Tnpo2 & -1.1 \\
\hline B-cell CLL/lymphoma 11A (zinc finger protein) & Bcl11a & -1.2 \\
\hline B-cell translocation gene 2 , anti-proliferative & Btg2 & -1.2 \\
\hline calmodulin regulated spectrin-associated protein & Camsap111 & -1.2 \\
\hline cyclin-dependent kinase inhibitor 2D & Cdkn2d & -1.2 \\
\hline $\operatorname{lamin} \mathrm{A}$ & Lmna & -1.2 \\
\hline
\end{tabular}


Table A.1 (continued).

\begin{tabular}{llc} 
Gene & Gene symbol & $\begin{array}{c}\text { Signal log ratio } \\
\text { (null/wt) }\end{array}$ \\
\hline aminolevulinic acid synthase 2, erythroid & Alas2 & -1.3 \\
biliverdin reductase B & Blvrb & -1.3 \\
STEAP family member 3 & Steap3 & -1.3 \\
synuclein, alpha & Snca & -1.5 \\
ankyrin repeat domain 9 & Ankrd9 & -1.7 \\
erythrocyte protein band 4.2 & Epb4.2 & -1.8 \\
aminolevulinate, delta-, dehydratase & Epb4.2 & -1.8 \\
aminolevulinate, delta-, dehydratase & Alad & -1.9 \\
eukaryotic elongation factor-2 kinase & Eef2k & -1.9 \\
E2F transcription factor 2 & E2f2 & -2.4 \\
erythrocyte protein band 4.1 & Epb4.1 & -2.4 \\
Kruppel-like factor 1 (erythroid) & Klf1 & -2.5 \\
ankyrin 1, erythroid & Ank1 & -2.6 \\
LIM domain only 2 & Lmo2 & -2.5 \\
Kruppel-like factor 3 (basic) & Klf3 & -2.8 \\
transferrin receptor & Tfr & -2.8 \\
SRY-box containing gene 6 & Sox6 & -3.4 \\
Duffy blood group & Dfy & -3.8 \\
erythrocyte protein band 4.9 & Epb4.9 & -5.1 \\
erythroid associated factor & Eraf; AHSP & -7.2 \\
\hline
\end{tabular}




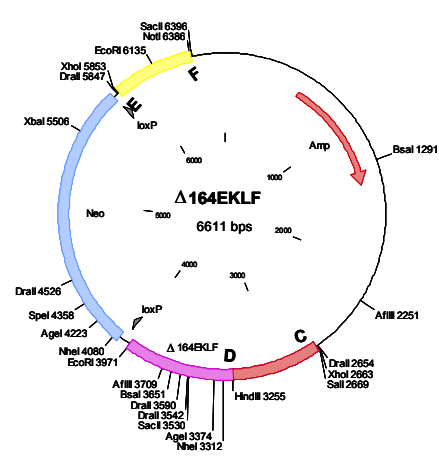

$\Delta 164 \mathrm{EKLF}$

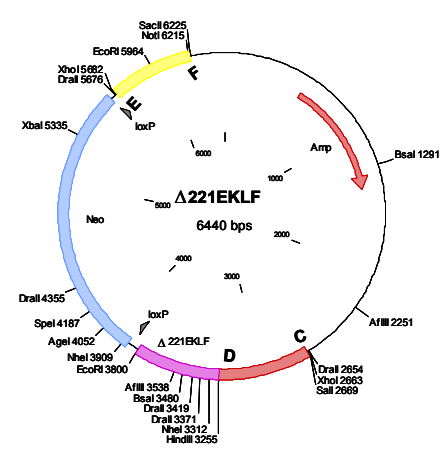

$\Delta 221 \mathrm{EKLF}$

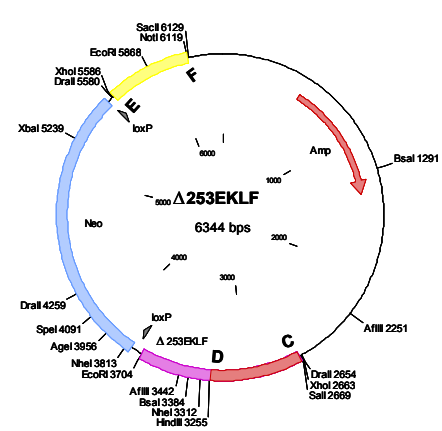

$\triangle 253 E K L F$

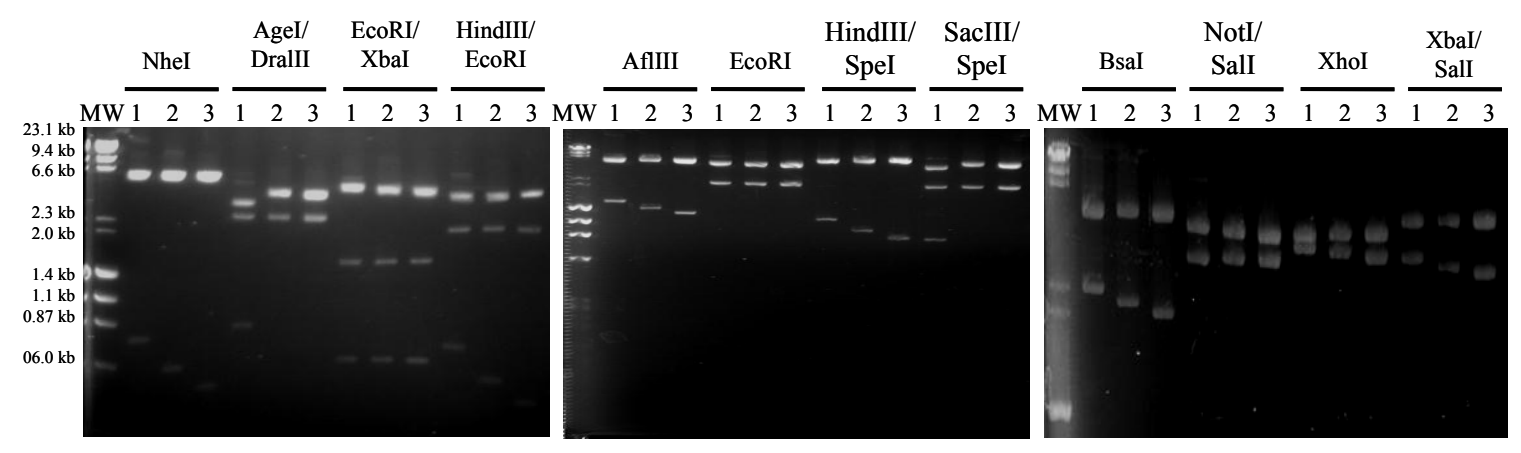

Figure A.1. Confirmation of the mini-targeting vectors. The top panel shows the plasmid maps of the mini targeting vectors, HA- $\triangle 164 \mathrm{EKLF}, \mathrm{HA}-\triangle 221 \mathrm{EKLF}$, and HA$\triangle 253 \mathrm{EKLF}$, respectively. The bottom panel is representative agarose gels after digestion of the mini targeting vectors with the restriction enzymes. Lane $1=\mathrm{HA}-\Delta 164 \mathrm{EKLF}$; lane $2=$ HA- $\Delta 221$ EKLF; lane $3=$ HA- $\Delta$ 153EKLF. The predicted fragments are given in Appendix Table A.2. 
Table A.2. Predicted fragment size with restriction enzyme digestion.

\begin{tabular}{|c|c|c|c|}
\hline Enzyme & $\Delta 164(1)$ & $\Delta 221(2)$ & $\Delta 253(3)$ \\
\hline NheI & $\begin{array}{l}5.8 \\
0.8\end{array}$ & $\begin{array}{l}5.8 \\
0.6\end{array}$ & $\begin{array}{l}5.8 \\
0.5\end{array}$ \\
\hline AgeI/DraIII & $\begin{array}{l}3.2 \\
2.6 \\
0.9\end{array}$ & $\begin{array}{l}3.8 \\
2.6\end{array}$ & $\begin{array}{l}3.7 \\
2.6\end{array}$ \\
\hline EcoRI/XbaI & $\begin{array}{l}4.4 \\
1.5 \\
0.6\end{array}$ & $\begin{array}{l}4.3 \\
1.5 \\
0.6\end{array}$ & $\begin{array}{l}4.2 \\
1.5 \\
0.6\end{array}$ \\
\hline HindIII/EcoRI & $\begin{array}{l}3.7 \\
2.2 \\
0.7\end{array}$ & $\begin{array}{l}3.7 \\
2.2 \\
0.6\end{array}$ & $\begin{array}{l}3.7 \\
2.2 \\
0.5\end{array}$ \\
\hline AflIII & $\begin{array}{l}5.2 \\
1.5\end{array}$ & $\begin{array}{l}5.2 \\
1.3\end{array}$ & $\begin{array}{l}5.2 \\
1.2\end{array}$ \\
\hline EcoRI & $\begin{array}{l}4.4 \\
2.2\end{array}$ & $\begin{array}{l}4.3 \\
2.2\end{array}$ & $\begin{array}{l}4.2 \\
2.2\end{array}$ \\
\hline HindIII/SpeI & $\begin{array}{l}5.5 \\
1.1\end{array}$ & $\begin{array}{l}5.5 \\
0.9\end{array}$ & $\begin{array}{l}5.5 \\
0.8\end{array}$ \\
\hline SacIII/SpeI & $\begin{array}{l}3.7 \\
2.0 \\
0.8\end{array}$ & $\begin{array}{l}4.4 \\
2.0\end{array}$ & $\begin{array}{l}4.3 \\
2.0\end{array}$ \\
\hline BsaI & $\begin{array}{l}4.3 \\
2.3\end{array}$ & $\begin{array}{l}4.3 \\
2.2\end{array}$ & $\begin{array}{l}4.3 \\
2.1\end{array}$ \\
\hline NotI/SalI & $\begin{array}{l}3.7 \\
2.9\end{array}$ & $\begin{array}{l}3.5 \\
2.9\end{array}$ & $\begin{array}{l}3.4 \\
2.9\end{array}$ \\
\hline XhoI & $\begin{array}{l}3.4 \\
3.2\end{array}$ & $\begin{array}{l}3.4 \\
3.0\end{array}$ & $\begin{array}{l}3.4 \\
2.8\end{array}$ \\
\hline $\mathrm{XbaI} / \mathrm{SalI}$ & $\begin{array}{l}3.8 \\
2.8\end{array}$ & $\begin{array}{l}3.8 \\
2.7\end{array}$ & $\begin{array}{l}3.8 \\
2.6\end{array}$ \\
\hline
\end{tabular}




\title{
A. 5' Southern blot probe
}

CTAGCTAAAGCCTTTCTTATTAATGCCAAAAGAACTGAAGCAGGAAATCA CGGTGGTTACACAGCCCAGCACCTAAGCCCAATTTACAGGACAAGGCTCA TTTTTATTATTAGAGCTCCTTTGGTAACAGAGAAGGCTGCTCACAGCCAGC ATGCTCAAGGTTGTATAAAGCACAACAATGTTATTTGTCTTCAGAAAAAC ATCTGAGGTGTGGGAACATCATTTACCCTGACTATGCT

\section{B. 3' Southern blot probe}

\begin{abstract}
AATTCGCCCTTAAGGGCCAGAACTTGGAGCGCCTTAGAAGTCACGTGACA GCCAATGCCCAAGGGAGAGGCGTGTCCACGGATGAAGCCTATCCGGAGC TGAGTCGGCCAGAAGCGGGAGGACGGGGCGGGGAGGACATTGGAGGAG GCGGGGTCCGCTTGTGGGACAACTTCATAGGAAGCCCCGCCTCCGTATTG GGCCTGGCGGGCTTCGTCTCCACCCTCGCGAAGCCGGGACACAGATCTCT TCGGTTTTTAGGGGAAGTTCATCTGAATCTCGTGACCCGGCTGTCCCCGGG TCTTTTAGAGTCCATTGCTGTCGCCTCATGGCAACACTGAGATCGCTGCTG CTGGCTGCGCTGCTGTGGGTCCCTGCCGAAGCCCTGAGCTGCTATGGGGA CTCCGGGCAGCCTGTGGATTGGTGAGTAAGTAGTCGCGGGACTGTCCCCC GCACACTGCCTGGGGACCGGCGCGGGAATCCAAAAAACCTCAGATTCCTT CTCTCCCCCAACCTCATGTCTTCACGGACCTCCAGGTTCGTGGT
\end{abstract}

Figure A.2. Sequences of Southern blot probes used in screening targeted ES cells and genotyping of animals. (A) The $239 \mathrm{bp} \mathrm{5'}$ probe was used with $E c o R V$ digest to

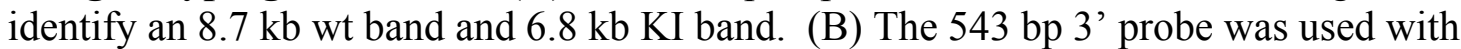
NheI digest to identify an $11.1 \mathrm{~kb}$ wt band and $9.1 \mathrm{~kb}$ KI band. 
Table A.3. Buffer composition used in ChIP analysis.

\section{Buffer}

Cell Lysis

Nuclei Lysis

IP Dilution Buffer

IP Wash Buffer 1

IP Wash Buffer 2

Elution Buffer

\section{Composition}

$10 \mathrm{mM}$ Tris $\mathrm{pH} 8.0$

$10 \mathrm{mM} \mathrm{NaCl}$

$0.2 \% \mathrm{NP} 40$

Add Protease inhibitors (Roche) and PMSF before use.

$50 \mathrm{mM}$ Tris $\mathrm{pH} 8.0$

$10 \mathrm{mM}$ EDTA

$1 \%$ SDS

Add Protease inhibitors (Roche) and PMSF before use.

$20 \mathrm{mM}$ Tris $\mathrm{pH} 8.0$

$2 \mathrm{mM}$ EDTA

$150 \mathrm{mM} \mathrm{NaCl}$

$1 \%$ Triton X-100

$0.01 \%$ SDS

Add Protease inhibitors (Roche) and PMSF before use.

$20 \mathrm{mM}$ Tris $\mathrm{pH} 8.0$

$2 \mathrm{mM}$ EDTA

$50 \mathrm{mM} \mathrm{NaCl}$

1\% Triton X-100

$0.1 \%$ SDS

10mM Tris $\mathrm{pH} 8.0$

$1 \mathrm{mM}$ EDTA

$0.25 \mathrm{M} \mathrm{LiCl}$

$1 \%$ NP40

$1 \%$ Deoxycholic acid

$100 \mathrm{mM} \mathrm{NaHCO} 3$

$1 \%$ SDS (use high quality SDS) 
Table A.4. Primers used for ChIP analysis.

\begin{tabular}{|c|c|c|}
\hline Region & Primer sequence & Reference \\
\hline HS2 & $\begin{array}{l}\text { 5'-AGTCAATTCTCTACTCCCCACCCT-3' } \\
\text { 5'-ACTGCTGTGCTCAAGCCTGAT-3' }\end{array}$ & Kiekhaefer et al., 2002 \\
\hline IVR & $\begin{array}{l}\text { 5'-GTATGCTCAATTCAAATGTACCTTATTTTAA-3', } \\
\text { 5'-TTACСТCTTTATTTCACTTTTACACATAGCTAA-3' }\end{array}$ & Kiekhaefer et al., 2002 \\
\hline$\beta$ maj pro & $\begin{array}{l}\text { 5'-CAGGGAGAAATATGCTTGTCATCA-3' } \\
\text { 5'-GTGAGCAGATTGGCCCTTACC-3' }\end{array}$ & Kiekhaefer et al., 2002 \\
\hline$\beta$ maj ex3 & $\begin{array}{l}\text { 5'-GCCCTGGCTCACAAGTACCA-3', } \\
\text { 5'-TTCACAGGCAAGAGCAGGAA-3' }\end{array}$ & Kiekhaefer et al., 2002 \\
\hline AHSP & $\begin{array}{l}\text { 5'-CTAACTCCAGGGAAGCCTCACC-3' } \\
\text { 5'-TTTGTGTGTCTTCTGCACTAAGCG-3' }\end{array}$ & Pilon et al., 2006 \\
\hline Fli-1 & $\begin{array}{l}\text { 5'-CGTGGACCCCGTCATTGTT-3' } \\
\text { 5'-GCACTGCGCACACAGGATACT-3' }\end{array}$ & Frontelo et al., 2007 \\
\hline RPII215 & $\begin{array}{l}\text { 5'-GCGAATCTATAAAGGGCGTCACT-3' } \\
\text { 5'-TCGGCGCTTCTGAGGAGA-3' }\end{array}$ & Kiekhaefer et al., 2002 \\
\hline$\alpha$-globin pro & $\begin{array}{l}\text { 5'-TGACCAAGGTAGGAGGATACTAACTTCT-3' } \\
\text { 5'-TTGCCCGGACACACTTCTTAC-3' }\end{array}$ & Anquita et al., 2004 \\
\hline
\end{tabular}


Table A.5. Primers used for real time RT-PCR analysis.

\begin{tabular}{|c|c|c|}
\hline Gene & Primer sequence & Reference \\
\hline Alas 2 & $\begin{array}{l}\text { 5'-CACCTATGCTTAAGGAGCCA-3' } \\
\text { 5'-CAGAAGCACACAGGAAAGCA-3' }\end{array}$ & Drissen et al., 2005 \\
\hline Alad & $\begin{array}{l}\text { 5'-CTTTGATCTCAGGACTGCTG-3' } \\
\text { 5'-AACAGCTGCGGTGCAAAGTA-3' }\end{array}$ & Drissen et al., 2005 \\
\hline Pbgd & $\begin{array}{l}\text { 5'-TACTTCTGGCTTCCAAGTGC-3' } \\
\text { 5'-CAAGGTGAGGCATATCTTCC-3' }\end{array}$ & Drissen et al., 2005 \\
\hline Urod & $\begin{array}{l}\text { 5'-ATCCCTGTGCCTTGTATGCA-3' } \\
\text { 5'-AGGTTGGCAATTGAGCGTTG-3' }\end{array}$ & Drissen et al., 2005 \\
\hline Cpox & $\begin{array}{l}\text { 5'-CAATTTGAAGCCAGTCCGTG-3' } \\
\text { 5'-CTGGACTAGAACTCCCTTTG-3' }\end{array}$ & Drissen et al., 2005 \\
\hline Ppox & $\begin{array}{l}\text { 5'-ATTCCAGCTTCAGAGCTCAG-3' } \\
\text { 5'-TACTGCAGATTCACCACAGC-3' }\end{array}$ & Drissen et al., 2005 \\
\hline Fech & $\begin{array}{l}\text { 5'-ACCAGTGACCACATTGAGAC-3', } \\
\text { 5'-GGCCTTGGAGAACAATGGAT-3' }\end{array}$ & Drissen et al., 2005 \\
\hline AHSP & $\begin{array}{l}\text { 5'-GGATCAGCAGGTCTTTGATG-3' } \\
\text { 5'-AGAGTACTCAGCTCTTGCTG-3' }\end{array}$ & Drissen et al., 2005 \\
\hline
\end{tabular}


Table A.5 (continued).

\begin{tabular}{|c|c|c|}
\hline Gene & Primer sequence & Reference \\
\hline Epb4.9 & $\begin{array}{l}\text { 5'-TGCTCAAGACCCAAGGCTTA-3' } \\
\text { 5'-TCCTATCTGGTTTTGCCTGG-3' }\end{array}$ & Drissen et al., 2005 \\
\hline GAPDH & $\begin{array}{l}\text { 5'-CCTGCCAAGTATGATGACAT-3' } \\
\text { 5'-GTCCTCAGTGTAGCCCAAG-3' }\end{array}$ & Drissen et al., 2005 \\
\hline Ankyrin & $\begin{array}{l}\text { 5'-CTCCAGCCGGACCTGATAGAG-3' } \\
\text { 5'-GAACACGTGCGACCCTTCAGTAG-3' }\end{array}$ & Nilson et al., 2006 \\
\hline Band 3 & $\begin{array}{l}\text { 5'-CACAGTGCCTCTCCGTCGTCTCATC-3' } \\
\text { 5'-CCTTCCCCACCCACAGCCATAACAC-3' }\end{array}$ & Nilson et al., 2006 \\
\hline BKLF & $\begin{array}{l}\text { 5'-GAAATGTCACCCCCTTTAATGAAC-3' } \\
\text { 5'-CACGATGACGGAAGGATGGT-3' }\end{array}$ & Funnell et al., 2007 \\
\hline Fli-1 & $\begin{array}{l}\text { 5'-CAACCAGCCAGTGAGAGTCA-3' } \\
\text { 5'-GCCCACCAGCTTGTTACATT-3' }\end{array}$ & \\
\hline$\beta$-globin mRNA & $\begin{array}{l}\text { 5'-TTTAACGATGGCCTGAATCACTT-3' } \\
\text { 5'-CAGCACAATCAGATCATATTGC-3' }\end{array}$ & \\
\hline$\beta$-globin $1^{\circ}$ transcripts & $\begin{array}{l}\text { 5'-СТТСТСТСТСТССТСТСТСТTТСТСТАATC-3' } \\
\text { 5'-AATGAACTGAGGGAAAGGAAAGG-3' }\end{array}$ & \\
\hline
\end{tabular}




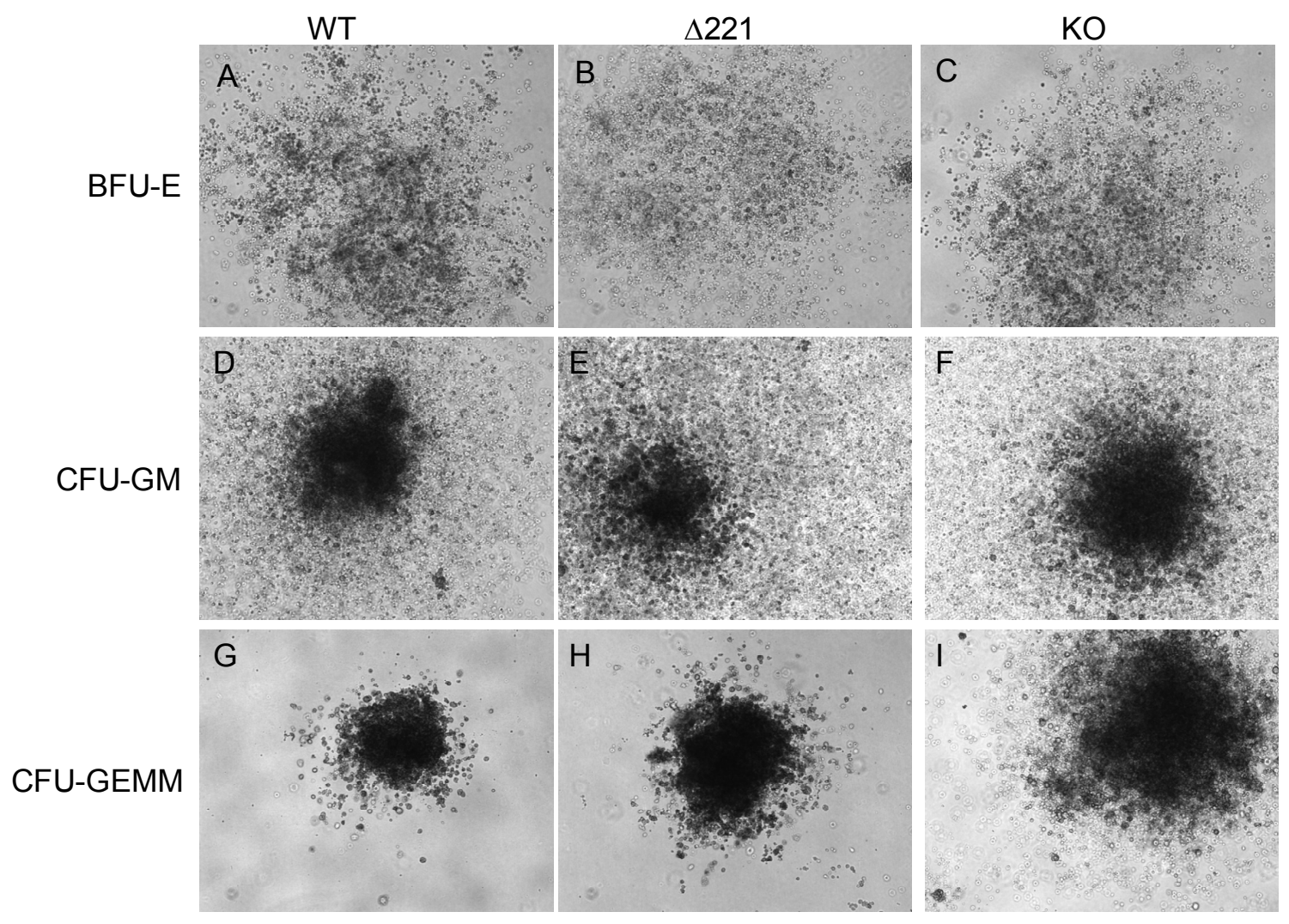

Figure A.3. Fetal liver hematopoietic progenitors. Fetal liver cells were plated in methocellulose medium and colonies were scored after 14 days of growth. Panels a-c: burst-forming unit-erythroid (BFU-E). Panels d-f: colony-forming unit-granulocytemonocyte (CFU-GM). Panels g-i: colony-forming unit-granulocyte-erythroid-monocytemegakaryocyte. 


\section{VITA}

Valerie Malyvanh Jansen was born Phoukhaokham Malyvanh on September 18, 1977, in Vientiane, Laos. She immigrated with her parents to the United States at the age of 6 years and grew up in Nashville, Tennessee. In 1997, she graduated from John Overton Comprehensive High School and was accepted as a Presidential Scholar at Maryville College in Maryville, Tennessee. As an undergraduate student, she completed an internship at Oak Ridge National Laboratory in Oak Ridge, Tennessee, under the supervision of B.R. Evans, $\mathrm{PhD}$, that resulted in a named U.S. patent. She graduated Magna Cum Laude with a BA in Chemistry in May 2001. In August 2001, she enrolled in medical school at the University of Tennessee Health Science Center in Memphis, Tennessee. In July 2003, she began her doctoral studies in the Department of Molecular Sciences, conducting her research at St. Jude Children's Research Hospital in the Division of Experimental Hematology under Dr. John M. Cunningham. In 2007, she moved with Dr. Cunningham's laboratory and completed her dissertation research at the University of Chicago, in Chicago, Illinois, in the Section of Pediatric Hematology/Oncology. Her PhD was granted in May 2009 from the University of Tennessee. She expects to graduate from Pritzker School of Medicine at the University of Chicago in June 2010. 